\title{
Rigidez quase-simétrica de mapas multicríticos do círculo
}

\author{
Gabriela A. Estevez Jacinto
}

\author{
TESE APRESENTADA \\ $\mathrm{AO}$ \\ Instituto DE MATEMÁTICA E EsTATístiCA \\ UNIVERSIDADE DE SÃO PAULO \\ PARA \\ OBTENÇÃO DO TÍTULO \\ DOUTORA EM MATEMÁTICA
}

\author{
Programa: Doutorado em Matemática \\ Orientador: Prof. Dr. Edson de Faria
}

Durante o desenvolvimento deste trabalho o autor recebeu auxílio financeiro da CAPES

São Paulo, março de 2017 


\section{Rigidez quase-simétrica de mapas multicríticos do círculo}

Esta é a versão original da tese elaborada pela candidata Gabriela Alexandra Estevez Jacinto, tal como submetida à Comissão Julgadora. 


\section{Rigidez quase-simétrica de mapas multicríticos do círculo}

Esta versão da tese contém as correções e alterações sugeridas pela Comissão Julgadora durante a defesa da versão original do trabalho, realizada em 10/03/2017. Uma cópia da versão original está disponível no Instituto de Matemática e Estatística da Universidade de São Paulo.

Comissão Julgadora:

- Prof. Dr. Edson de Faria (orientador) - IME-USP

- Prof. Dr. Pablo Guarino - UFF

- Prof. Dr. Daniel Smania - ICMC-USP

- Prof. Dr. Kleyber Cunha - UFBA

- Prof. Dr. Sylvain Bonnot - IME-USP 


\section{Agradecimentos}

Quero agradecer ao meu orientador Edson de Faria, por ter me aceitado como sua aluna, pela confiança que teve durante todos estes anos, pela paciência a generosidade de compartilhar tanto conhecimento. Agradeço também ao Pablo Guarino, meu co-orientador não oficial, pela paciência, sua generosidade, por estar sempre disposto a me ajudar e pelas conversas sob a vida matemática e não matemática.

A mis padres, Ysabel y Luis, a mis hermanas, Carolina y Luisa, por acompañarme y respaldarme en todas las decisiones de mi vida, por escucharme y encontrar la forma de levantarme el ánimo en los momentos difíciles. Este trabajo es para ustedes y por ustedes.

A meus professores da Colômbia que me permitiram chegar a USP, Serafin Bautista e Felix Soriano. Aos professores Welington de Melo (Q.E.P.D.), Charles Tresser, Edson Vargas, Andre de Carvalho, Albert Fisher, Sylvain Bonnot, Luna Lomonaco, Carlos Siqueira, Arlane Silva Vieira, Marcelo Viana, Alexander Arbieto, Daniel Smania, Maria José Pacífico, Patricia Cirilo, Pablo Carrasco, Kleyber Cunha. Especialmente ao professor Enrique Pujals, por compartilhar seus conhecimentos, pelos seminários e pelas perguntas que sempre me fez.

Ao meus amigos em São Paulo, em especial a Daniel, Chauta, Laura, Elkin, German, Dionicio, Diego, Diana, Alex, Genaro e Denisse. Aos meus post-docs favoritos: Rafa e Felipe. Ao Julio, pelo carinho,

o imenso apoio, obrigada também pela ajuda com os gráficos. Ao meus amigos no Rio, especialmente a Caro, Sebastien, Plinio, Catalina, Mariela, Dieguito, Bruno, Yuri.

Aos Professores membros da banca examinadora, por aceitar o convite.

Agradeço ao CAPES pelo suporte financeiro durante o Doutorado. Ao IMPA por sua hospitalidade e suporte financeiro durante os cursos de verão e durante o semestre temático. Ao IME e seus funcionários pela ajuda com respeito as questões burocráticas e técnicas. 


\section{Resumo}

Estevez-Jacinto, Gabriela A. Rigidez quase-simétrica de mapas multicríticos do círculo. 2017. 120 f. Tese (Doutorado) - Instituto de Matemática e Estatística, Universidade de São Paulo, São Paulo, 2017.

No presente trabalho consideramos homeomorfismos do círculo sem pontos periódicos e com o mesmo número finito de pontos críticos todos de tipo non-flat. Provamos que se existe uma conjugação topológica entre dois destes mapas que leva ponto crítico em ponto crítico, sem necessidade de preservar criticalidades, então dita conjugação é uma transformação quase-simétrica com distorção quase-simétrica local uniformemente limitada. Estes resultados são válidos para qualquer número de rotação irracional e são independentes da natureza das criticalidades dos pontos críticos, de modo que nossos resultados são válidos para toda criticalidade real.

Palavras-chave: Mapas multicríticos do círculo, Real Bounds, partições dinâmicas, rigidez. 


\section{Abstract}

Estevez-Jacinto, Gabriela A. Quasisymmetric rigidity of multicritical circle maps. 2017. $120 \mathrm{f}$. Tese (Doutorado) - Instituto de Matemática e Estatística, Universidade de São Paulo, São Paulo, 2017.

In this work we consider circle homeomorphisms without periodic points and with finite number of critical points all of them being non-flat. We prove that if there exists a topological conjugacy between two of those maps which sends critical point into critical point, which not necessarily preserve criticalities, then this conjugacy is a quasi-symmetric map with quasi-symmetric distortion universally bounded. All these results are valid for any irrational rotation number and are independent of the nature of the criticalities, therefore our results are valid for all real criticalities.

Keywords: Multicritical circle maps, Real Bounds, dynamical partitions, rigidity. 


\section{Sumário}

1 Introdução 1

1.1 Como está organizada esta tese . . . . . . . . . . . . . . . . . . . 4

2 Preliminares $\quad 5$

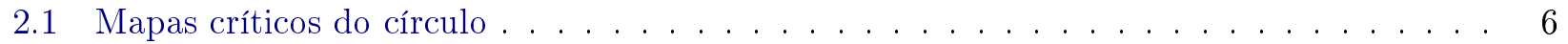

2.2 Mapas multicríticos do círculo . . . . . . . . . . . . . . . . . . . 7

2.2.1 Propriedades combinatórias . . . . . . . . . . . . . . . . 9

2.2 .2 Propriedades topológicas . . . . . . . . . . . . . . . . 11

2.2 .3 Propriedades ergódicas . . . . . . . . . . . . . . . . . . . 12

2.2 .4 Propriedades de regularidade . . . . . . . . . . . . . . . . . . 12

2.3 Pares multicríticos comutativos . . . . . . . . . . . . . . . . . . 14

2.4 Renormalização de pares multicríticos comutativos . . . . . . . . . . . . . . 16

3 Rigidez quase-simétrica de mapas multicríticos do círculo $r$

3.1 Geometria das partições dinâmicas . . . . . . . . . . . . . . . . . . 20

3.1.1 Intervalos que se intersectam são comparáveis . . . . . . . . . . . . . . . 21

3.1 .2 Os intervalos críticos são longos . . . . . . . . . . . . . . . . . 21

3.1 .3 Uma partição auxiliar . . . . . . . . . . . . . . . . . . . 23

3.1 .4 Mapas quase-parabólicos . . . . . . . . . . . . . . . . 25

3.1.5 Decomposição balanceada de pontes . . . . . . . . . . . . . . . . . 29

3.2 Prova do Teorema da conjugação quase-simétrica . . . . . . . . . . . . . . . . . . . 33

3.2 .1 Uma fine grid adequada . . . . . . . . . . . . . . . . . 33

3.2 .2 Prova do Teorema $3.0 .1 \ldots \ldots \ldots \ldots \ldots$

3.2.3 Comentários sob a quase-simetria para mapas com quantidade diferente de pontos

críticos . . . . . . . . . . . . . . . . . 35

4 Cotas beau para mapas multicríticos do círculo $\quad 37$

4.1 As $\operatorname{cotas} C^{1} \ldots \ldots \ldots \ldots \ldots \ldots \ldots \ldots \ldots$

4.1 .1 Prova do Lema $4.1 .1 \ldots \ldots \ldots \ldots \ldots$

4.2 Derivada Schwarziana negativa do mapa de primeiro retorno . . . . . . . . . . . . . 40

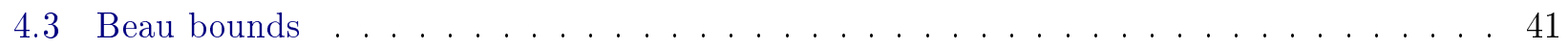

4.3 .1 Decomposição dos iterados de $f \ldots \ldots \ldots \ldots$. . . . . . . . . . . 42

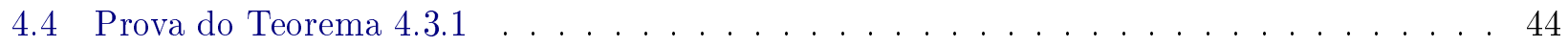

5 Considerações finais $\quad 45$ 
$\begin{array}{ll}\text { A Mapas do círculo } & 47\end{array}$

A.1 Número de rotação . . . . . . . . . . . . . . . . . . . . . . . . . . 47

A.2 Cross-ratio e derivada Schwarziana . . . . . . . . . . . . . . . . 51

A.3 Pontos críticos non-flat . . . . . . . . . . . . . . . . . . . 54

$\begin{array}{ll}\text { B Prova do Teorema 2.2.2 } & \mathbf{5 7}\end{array}$

B.1 Comparabilidade de intervalos dinâmicamente simétricos . . . . . . . . . . . . 57

B.2 Comparabilidade de seis intervalos dinâmicos . . . . . . . . . . . . . . . 60

B.3 Prova do Teorema $2.2 .2 \ldots \ldots \ldots \ldots \ldots \ldots \ldots$

C Mapas quase-simétricos $\quad 65$

C.1 Prova da Proposição $3.0 .1 \ldots \ldots \ldots \ldots \ldots$. . . . . . . . . . . . 66

$\begin{array}{lr}\text { Referências Bibliográficas } & 69\end{array}$ 


\section{Capítulo 1}

\section{Introdução}

O fenômeno de rigidez acontece quando uma equivalência débil entre classes de sistemas dinâmicos se torna uma equivalência forte sob certas condições. Talvez um dos resultados mais célebres nesta direção é o teorema de rigidez de Mostow, é um dos resultados mais influentes dos últimos cinquenta anos em geometria e topologia.

Na teoria da dinâmica unidimensional, o fenômeno da rigidez aparece primeiro no cenário dos difeomorfismos do círculo. Mais precisamente, em [Her79] Herman provou que existe um conjunto de medida total $B \subseteq[0,1]$, tal que todo difeomorfismo $C^{k}$ do círculo com $k \geq 3$ cujo número de rotação pertence a $B$, é conjugado à correspondente rotação por um difeomorfismo $C^{k-2}$. Veja também Yoccoz [Yoc84a].

Anos mais tarde, Stark [Sta88], Khanin e Sinai [KS87], [KS89], Rand [Ran88] e Katznelson e Ornstein [YK89], simplificaram a prova do teorema de Herman utilizando métodos de renormalização: se $f$ é um difeomorfismo do círculo de classe $C^{r}$, com $r \geq 3$, e seu número de rotação $\rho$ satisfaz a condição Diofantina

$$
\left|\rho-\frac{p}{q}\right| \geq \frac{C}{q^{2+\beta}}
$$

para todos os números racionais $p / q$, para algumas constantes $C>0$ e $0 \leq \beta<1$, então $f$ é $C^{r-1-\beta-\epsilon_{-}}$ conjugada à correspondente rotação rígida, para todo $\epsilon>0$. Portanto, para quase todos os números de rotação, um difeomorfismo do círculo suficientemente suave é suavemente conjugado à rotação correspondente. A pequena perda da diferenciabilidade da conjugação é inerente a problemas de pequenosdenominadores, os quais estão presentes inclusive se o difeomorfismo em questão é uma perturbação pequena de uma rotação.

Em 1961, Arnol'd [Arn61] provou que todo difeomorfismo analítico do círculo com número de rotação de tipo Diofantino é conjugado por um homeomorfismo analítico à rotação rígida correspondente, sempre que o difeomorfismo for suficientemente próximo da rotação, e conjecturou que a hipótese de proximidade entre o mapa e a rotação correspondente não era necessária para que o resultado fosse verdadeiro. Nesse mesmo trabalho, Arnol'd provou que existem difeomorfismos do círculo analíticos reais que não são sequer absolutamente continuamente conjugados à rotação correspondente. Estes resultados (reforçados por novos desenvolvimentos, e.g., [YK89] e [KT09]) produzem uma solução bastante completa ao problema de rigidez para difeomorfismos do círculo.

O fenômeno da rigidez também aparece no caso dos mapas críticos do círculo, i.e. homeomorfismos do círculo que preservam orientação com um número finito de pontos críticos de tipo non-flat. Estes mapas pertencem à fronteira entre difeomorfismos e endomorfismos do círculo, i.e. entre entropia 
topológica zero e entropia topológica positiva. Este tipo de mapas tem sido estudados por muitos matemáticos e físicos devido às suas aplicações nas ciências naturais. Por exemplo, aparecem no estudo dos fluidos como transição para a turbulência através da intermitência [PM80], no estudo da arritmia cardíaca [Gla91], dispositivos eletrônicos [BBJ84], entre outros.

Pelo trabalho de Yoccoz [Yoc84b], dois mapas do círculo com mesmo número de rotação irracional são topologicamente conjugados. A condição de non-flat sobre pontos críticos é necessária: Em [Hal81], Hall construiu um homeomorfismo do círculo $C^{\infty}$ com pelo menos um ponto crítico de tipo flat que não possui pontos periódicos nem órbitas densas.

Como a conjugação entre um mapa crítico e a rotação não pode ser suave, então o estudo da rigidez se concentra na classe dos mapas críticos do círculo. Alguns resultados de rigidez foram obtidos, no caso de um único ponto crítico, todos estes usando o operador de renormalização. Quando os mapas são analíticos reais, de Faria and de Melo [dFdM00] provaram que dois de estes mapas do círculo com mesmo número de rotação de tipo limitado (isto é, $\beta=0$ na desigualdade (1.0.1)) são $C^{1+\alpha}$ conjugados para algum $0<\alpha<1$. Mais tarde, em [KT07], Khanin e Teplinsky provaram que dois mapas críticos do círculo, analíticos reais, com o mesmo número (arbitrário) de rotação irracional são conjugados por um difeomorfismo $C^{1}$.

Quando os mapas são suaves, de Faria e de Melo em [dFdM99] provaram que se as renormalizações sucessivas de dois mapas de críticos do círculo $C^{3}$ convergem exponencialmente rápido na topologia $C^{0}$, então a conjugação é um difeomorfismo $C^{1+\alpha}$ para algum $0<\alpha<1$, sempre que o número de rotação pertença a um determinado conjunto $\mathbb{A}$ de números de rotação com medida de Lebesgue total. $\mathrm{O}$ conjunto $\mathbb{A}$ contém os números de tipo limitado, alguns números Diofantinos (mas não todos) e alguns números Liouville.

A condição sob a renormalizações foi provada por de Melo e Guarino em [GdM13] para o caso número de rotação de tipo limitado. Asim, dois mapas críticos do círculo $C^{3}$ com mesmo número de rotação de tipo limitado e com único ponto crítico (da mesma criticalidade impar), são conjugados por um difeomorfismo do círculo de classe $C^{1+\alpha}$, para algum $\alpha>0$ universal. Finalmente, em [GMdM15], de Melo, Guarino e Martens provaram o mesmo resultado para os números de rotação que pertencem ao conjunto $\mathbb{A}$ definido antes: quaisquer dois mapas críticos do círculo $C^{4}$ com o mesmo número de rotação pertencem a $\mathbb{A}$ e com único ponto crítico (novamente da mesma criticalidade ímpar) são conjugados por um difeomorfismo do círculo $C^{1+\alpha}$.

A condição sob o número de rotação é necessária. Em [dFdM99] os autores forneceram contraexemplos de mapas críticos do círculo $C^{\infty}$ cujo número de rotação irracional não pertence ao conjunto $\mathbb{A}$, tal que a conjugação entre eles não pode ser $C^{1+\alpha}$, para qualquer $0<\alpha<1$. Em [Avi13], Avila usou a renormalização parabólica para construir exemplos de mapas críticos do círculo analíticos reais que não podem ser $C^{1+\alpha}$ conjugados, para qualquer $0<\alpha<1$.

Todos esses resultados anteriores mostram que a teoria da rigidez está quase completa no caso de um unico ponto crítico. Em contraste, para mapas com mais de um ponto crítico, ou mapas de círculos multicríticos, a teoria da rigidez está longe de estar completa. Neste trabalho, nós lidamos com estes tipos de mapas. Provamos alguns resultados que no caso de um único crítico são verdadeiros.

Para formular adequadamente nosso primeiro resultado precisamos da seguinte noção: se $h: S^{1} \rightarrow$ $S^{1}$ é um homeomorfismo do círculo que preserva orientação, definimos sua distorção quase-simétrica 
local como a função $\sigma_{h}: S^{1} \rightarrow \mathbb{R}^{+} \cup\{\infty\}$ dada por

$$
\sigma_{h}(x)=\lim _{\delta \rightarrow 0} \limsup _{|t| \leq \delta} \frac{|h(x+t)-h(x)|}{|h(x)-h(x-t)|} .
$$

Quando $\sigma_{h}(x) \leq M$ para todo $x \in S^{1}$ e alguma constante $M \geq 1$, dizemos que $h$ é quase-simétrica.

Nosso primeiro resultado é o seguinte

Teorema A. [EdF15] Sejam $f, g: S^{1} \rightarrow S^{1}$ dois mapas multicríticos do círculo de classe $C^{r}(r \geq 3)$ com mesmo número de rotação irracional e o mesmo número de pontos críticos, todos eles de tipo non-flat. Se $h: S^{1} \rightarrow S^{1}$ é uma conjugação entre $f$ e g que leva ponto crítico em ponto crítico então $h$ é uma transformação quase-simétrica.

A prova do teorema A está baseada na prova do resultado análogo para o caso de mapas uni-críticos do círculo dada em [dFdM99]. Portanto, na prova só usamos métodos de análise real e o resultado é valido para criticalidades não inteiras dos pontos críticos. Notamos também que a conjugação $h$ não precisa preservar as criticalidades dos pontos críticos. É importante destacar que quando as criticalidades são números inteiros, o teorema A é um caso especial de um teorema anunciado por T. Clark e S. van Strien em [CvS14], cuja prova usa ferramentas de análise complexa.

Um resultado de rigidez similar para mapas do círculo com intervalo flat foi provado por Palmisano em [Pal15]: dois mapas do círculo $C^{2}$ com intervalo flat são quase-simetricamente conjugados em seus conjuntos não-errantes, sempre que seu número de rotação seja de tipo limitado e que certas partições do círculo satisfaçam uma hipótese de geometria limitada.

Seja $f$ um mapa multicrítico do círculo e $c$ um ponto crítico. Sejam $\left\{q_{n}\right\}_{n \geq 0}$ a sequência de primeiros retornos da órbita de $c$, e para todo $n \geq 0$, seja $I_{n}(c)$ o intervalo com pontos extremais dados por $c$ e $f^{q_{n}}(c)$, que contém o ponto $f^{q_{n+2}}(c)$. O raio dos comprimentos

$$
s_{n}(c, f)=\frac{\left|I_{n+1}(c)\right|}{\left|I_{n}(c)\right|}
$$

é chamado a n-ésima proporção de escala de $f$ associada ao ponto crítico $c$ ou o $n$-ésimo scaling ratio de $f$ ao redor do ponto crítico $c$.

Um passo preliminar no estudo da rigidez $C^{1+\alpha}$, é obter cotas reais a-priori na geometria das órbitas dos pontos críticos. Como acontece em mapas unidimensionais com um único ponto crítico, o comportamento da órbita do ponto crítico determina o comportamento de todas as outras órbitas. Portanto, a tarefa reduz-se a encontrar cotas a-priori nas órbitas críticas, para isto é suficiente obter cotas uniformes na sequencia das proporções de escala (ou scaling ratios) ao redor de cada ponto crítico. Cotas deste tipo são chamadas de Beau Bounds pelo Sullivan em [Sul88]. Como no caso de mapas com um único ponto crítico, as beau bounds devem produzir uma forma forte de compacidade da renormalização de um determinado mapa multicrítico do círculo.

O seguinte teorema é o segundo resultado obtido na tese.

Teorema B. [EdFG16] Seja $f: S^{1} \rightarrow S^{1}$ um mapa multicrítico do círculo com número de rotação irracional. Então as sucessivos proporçôes de escala ao redor de cada um de seus pontos críticos são uniformemente limitados, e a cota é assintoticamente independente de $f$. 
Um corolário importante do Teorema B, juntamente com o Teorema A, é o seguinte resultado

Corolário 1.0.1. Sejam $f, g$ dois mapas multicríticos do círculo com mesmo número de rotação irracional e mesmo número de pontos críticos. Seja $h: S^{1} \rightarrow S^{1}$ uma conjugação entre $f$ e $g$ que leva ponto crítico em ponto crítico, então h é quase-simétrica, e sua distorção quase-simétrica local é universalmente limitada, i.e. existe uma constante $K>1$, que depende da quantidade de pontos críticos $e$ da maior criticalidade, tal que $\sigma_{h}(x) \leq K$ para todo $x \in S^{1}$.

\subsection{Como está organizada esta tese}

No capítulo 2, introduzimos o conceito de mapas multicríticos do círculo e mostramos suas propriedades mais relevantes. Além disso, introduzimos a noção de par multicrítico comutativo, o qual permite definir um operador de renormalização para mapas multicríticos do círculo.

A primeira contribuição da tese começa no capítulo 3, o qual contém a prova do Teorema A. Esta prova segue da construção de uma sequencia de partições auxiliares, que são refinamentos intermediários da partição dinâmica associada a um ponto crítico. Estas partições auxiliares vão permitir definir um fine grid (como foram definidas originalmente em [dFdM99]), que não é mais que uma sequência de novas partições do círculo que satisfazem certas condições. Existe um critério, estabelecido em [dFdM99], sobre a conjugação entre dois mapas multicríticos do círculo: se tal conjugação produzir um isomorfismo entre as fine grids dos dois mapas, então ela é quase-simétrica.

O capítulo 4 é dedicado à prova do Teorema B. A prova esta baseada numa decomposição adequada dos iterados do mapa multicrítico do círculo. Para isto, estabelecemos as cotas $C^{1}$ para os mapas de primeiro retorno em torno dos pontos críticos e provamos que sua derivada Schwarziana é negativa.

No capítulo 5, apresentamos algumas perguntas concernentes à existência ou não de uma conjugação suave entre dois mapas multicríticos do círculo.

O Apêndice A contém as provas dos fatos mais relevantes enunciados nos capítulos anteriores. Finalmente, no Apêndice B, estabelecemos as cotas a-priori reais para mapas multicríticos do círculo. Este tipo de cotas mostram que as partições dinâmicas de mapas multicríticos do círculo possuem geometria limitada. Este resultado é conhecido, em uma forma ligeiramente diferente, do trabalho não publicado de Herman [Her88], o qual esta baseado num trabalho prévio de Swiatek [Swi88]. Uma outra aproximação ao trabalho de Herman, pode ser achada no trabalho de Petersen [Pet00]. Ainda assim, fornecemos uma prova independente que em sua vez esta baseada naquela encontrada em [dFdM99] para mapas uni-críticos do círculo. 


\section{Capítulo 2}

\section{Preliminares}

Identificamos $S^{1}$ com o quociente $\mathbb{R} / \mathbb{Z}$, sob o mapa de cobrimento universal $\pi: \mathbb{R} \rightarrow S^{1}$ dado por $\pi(x)=\exp (2 \pi i x)$. Também identificamos $S^{1}$ como o grupo multiplicativo dos números complexos $S^{1}=\{z \in \mathbb{C}:|z|=1\}$.

Pela teoria desenvolvida pelo Poincaré [Poi82], a dinâmica do homeomorfismo $f$ do círculo depende do número de rotação $\rho(f)$ : a existência de órbitas periódicas é equivalente a $\rho(f) \in \mathbb{Q}$, e se $\rho(f)$ é um número irracional $f$ não tem órbitas periódicas. Como toda órbita da rotação irracional $R_{\rho}(x)=x+\rho$ é densa, um homeomorfismo do círculo $f$ é conjugado à rotação correspondente $R_{\rho(f)}$ se e somente se o seu conjunto minimal $K$ é igual a $S^{1}$ (veja Proposição A.1.2 no Apêndice A). Além disso, $f$ e $R_{\rho(f)}$ são conjugados se e somente se não existe um intervalo $J$ tal que $J, f(J), f^{2}(J), \cdots$ são dois a dois disjuntos: se $f$ e $R_{\rho(f)}$ são conjugadas não existe um intervalo $J$ tal que todos seus iterados $f^{i}(J) i \geq 0$, são disjuntos. Se $f$ não é conjugado com a rotação $R_{\rho(f)}$ então $K \neq S^{1}$ e toda componente conexa $I$ de $S^{1} \backslash K$ satisfaz que seus iterados $f^{i}(I)$, para $i \in \mathbb{N}$, são dois a dois disjuntos (veja [dMvS93, Capítulo I, página 34]).

Um intervalo $J$ tal que todos seus iterados $f^{i}(J) i \geq 0$, são dois a dois disjuntos é um intervalo errante. Assim, a conjugação topológica entre $f$ e a rotação correspondente depende da não existência de intervalos errantes.

Aumentando a diferenciabilidade dos mapas do círculo com número de rotação irracional, obtemos o seguinte resultado devido a Denjoy.

Teorema 2.0.1 (Teorema de Denjoy). [Den32] Todo difeomorfismo $C^{2}$ do círculo com número de rotação irracional é topologicamente conjugado à correspondente rotação rígida.

De fato, o resultado original de Denjoy é para $f$ difeomorfismo $C^{1}$ do círculo tal que $\log D f$ tem variação limitada ${ }^{1}$. Notemos que se $f$ é um difeomorfismo $C^{2}$ então $\log D f$ é de variação limitada tomando $V(f) \geq \int_{S^{1}}\left|D^{2} f(x)\right| /|D f(x)| d x$.

A hipótese sob a derivada do mapa é essencial, em [Den32], Denjoy também construiu exemplos de difeomorfismos $C^{1}$ com número de rotação irracional que possuem intervalos errantes, i.e. não são topologicamente conjugados à correspondente rotação, [dMvS93, Capítulo 1, Teorema 2.3].

\footnotetext{
${ }^{1}$ Existe uma constante $V(f)>0$ tal que dada qualquer partição finita ordenada do círculo $\left\{x_{0}, x_{1}, \cdots, x_{n}\right\}$ temos que: $\sum_{i=0}^{n-1}\left|\log D f\left(x_{i+1}\right)-\log D f\left(x_{i}\right)\right| \leq V(f)$.
} 
Como o estudo do espaço dos difeomorfismos do círculo está bem desenvolvido, continuamos com o estudo de mapas com pontos críticos. Para isto teríamos que estudar todos os tipos de pontos críticos existentes, por exemplo, pontos críticos que são máximos o mínimos locais ou pontos críticos que são pontos de inflexão. No primeiro casso, o mapa não é injetivo, i.e. é um endomorfismo do círculo. Nesta tese estamos interessados em estudar a dinâmica de mapas injetores com pontos críticos inflexivos. Estes mapas tem sido amplamente estudados por exemplo em [Yoc84b], [dF99], [dFdM99], [dFdM00], [Avi13], [Swi88], [GMdM15], [KY06], [Yam01], [Yam02], [Yam03] ou [CvS14].

\subsection{Mapas críticos do círculo}

Um mapa crítico do círculo é um homeomorfismo $f$ do círculo de classe $C^{3}$ que preserva orientação, com número de rotação irracional e que possui um único ponto crítico de tipo non-flat (veja apêndice A secção A.3).

A seguir, mostramos um exemplo de este tipo de mapas. Este exemplo pertence à Família de Arnol'd definida por Arnol'd em [Arn61] a qual é a família estândar de mapas do círculo.

Exemplo 2.1.1. [Familia de Arnol'd]

Seja $a \in[0,1)$ e $b \geq 0$. Definimos para todo $x \in \mathbb{R}$

$$
F_{a, b}(x)=x+a-\left(\frac{b}{2 \pi}\right) \sin (2 \pi x) .
$$

$F_{a, b}$ é um levantamento de um mapa do círculo $f_{a, b}$. A escolha do parâmetro b determina o tipo de mapa.

Para $\boldsymbol{b}=\boldsymbol{O}, f_{a, 0}$ é a família de rotaçôes rígidas $R_{a}(x)=x+a$.

Para $\boldsymbol{O}<\boldsymbol{b}<\mathbf{1}$, todo mapa na família pertence ao conjunto aberto dos difeomorfismos reais-analiticos. O conjunto de valores dos parâmetros correspondentes a mapas que tem um atrator hiperbólico periódico é um conjunto aberto e denso. De fato, é a união de um número contável de regiões disjuntas abertas chamadas de linguas de Arnol'd. Toda língua é limitada por um par de curvas suaves que convergem a um valor racional de a quando b vai para zero. Os mapas pertencentes as línguas são estruturalmente estáveis e possuem um único ponto periódico atrator. O complemento das línguas esta formado por curvas suaves que são o bordo da língua ou é uma curva que converge a um valor irracional de a quando b vai para zero.

Para $\boldsymbol{b}=1$, cada mapa da família é um homeomorfismo com um único ponto crítico inflexivo de grau 3 (veja a definição de grau no apêndice A secção A.3). O conjunto dos parâmetros a tal que o número de rotação de $f_{a, 1}$ é irracional tem medida de Lebesgue nula. A sua dimensão de Hausdorff é estritamente menor do que 1 e maior ou igual a $1 / 3$.

Para $\boldsymbol{b}>\mathbf{1}$, o mapa $f_{a, b}$ não é mais invertível e o seu número de rotação pode não existir e quando existir pode depender do ponto.

Veja mais observações em [dM11].

Assim, os mapas $f_{a, 1}$ com número de rotação irracional são exemplos dos mapas críticos nos quais estamos interessados. 
Seja $f$ um mapa crítico do círculo, com único ponto crítico, usando a combinatória do número de rotação de $f$ e estimações sob o cross-ratio degenerado Yoccoz provou em [Yoc84b] que não existem intervalos errantes para $f$. Como já sabemos, isto indica que $f$ e $R_{\rho(f)}$ são topologicamente conjugados. Para uma versão em inglês da prova do Teorema de Yoccoz, veja [Gua12, Apêndice A] ou [Gua15].

A hipótese sob a natureza do ponto crítico é inviolável para que o resultado de Yoccoz ainda seja válido, em [Hal81] Hall construiu um homeomorfismo críticos $C^{\infty}$ do círculo sem pontos periódicos e sem órbitas densas.

A combinatória de estes mapas críticos é a mesma que da correspondente rotação. Assim sua entropia topológica é nula e estes mapas são unicamente ergódicos (veja apêndice A). Como a conjugação entre um mapa crítico e a correspondente rotação não pode ser suave, então o estudo das propriedades geométricas de estes mapas se enfoca em estudar a conjugação entre dois mapas críticos do círculo. No caso de um único ponto crítico acontece que, sob algumas restrições topológicas suaves, a conjugação entre dois mapas críticos é diferenciável (e inclusive com derivada Holder) assim que ela existir. Veja por exemplo: [Yoc84b], [dF99], [dFdM99], [dFdM00], [Avi13], [Swi88], [GMdM15], [KY06], [Yam01], [Yam02], [Yam03] ou [CvS14].

Retornando ao teorema de Yoccoz, temos o seguinte

Observação 2.1.1. Na prova do teorema de Yoccoz, os mapas possuem um número finito de pontos críticos todos eles de tipo non-flat.

Assim, estamos interessados em estudar a conjugação entre dois mapas críticos do círculo com mais de um ponto crítico.

\subsection{Mapas multicríticos do círculo}

Definição 2.2.1. Um mapa multicrítico do círculo é um homeomorfismo $f$ do círculo de classe $C^{3}$ que preserva orientação e que possui $N=N(f)>1$ pontos críticos todos eles de tipo non-flat: cada ponto crítico c possui uma vizinhança $W$ tal que para todo $x \in W$, temos $f(x)=(\phi(x))^{d}+f(c)$ onde $\phi: W \rightarrow \phi(W)$ é um difeomorfismo local $C^{3} \operatorname{com} \phi(c)=0$.

Um exemplo de mapa multicrítico do círculo é obtido modificando a família de Arnol'd.

Exemplo 2.2.1. (Veja [Gua15]) Consideramos o seguinte mapa na reta inspirado na familia de Arnol'd.

$$
\tilde{f}_{a}(x)=x+a-\left(\frac{1}{2 N \pi}\right) \sin (2 N \pi t), \quad \text { para } a \in[0,1) \text { e } N \in \mathbb{N} .
$$

Como $\tilde{f}_{a}(x+1)=\tilde{f}_{a}(x)+1$ entâo $\tilde{f}_{a}$ é um levantamento pela projeção $\pi(t)=\exp (2 \pi i t)$ de um homeomorfismo do círculo, que vamos chamar $f_{a}: S^{1} \rightarrow S^{1}$.

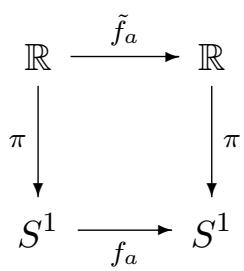


Notemos que $D \tilde{f}_{a}(x)=1-\cos (2 \pi N x)$ e portanto, $D \tilde{f}_{a}(x)=0(\bmod 1)$ se e somente se $x \in$ $\left\{0, \frac{1}{N}, \frac{2}{N}, \cdots, \frac{N-1}{N}\right\}$. Os pontos críticos do mapa $f_{a}$ estão dados pelos pontos $c_{k}=\exp \left(2 \pi i x_{k}\right)$ onde $x_{k}=\frac{k}{N}$ para $k \in\{0,1, \cdots, N-1\}$. É fácil ver que o grau de todos estes pontos críticos é igual a 3.

Como mencionamos na observação 2.1.1, Yoccoz [Yoc84b] provou que todo mapa multicrítico do círculo é minimal.

Teorema 2.2.1 (Teorema de Yoccoz). [Yoc84b] Todo mapa multicrítico do círculo, com número de rotação irracional, é topologicamente conjugado à correspondente rotação rígida, i.e. existe um homeomorfismo que conjuga estes dois mapas.

As seguintes são propriedades dos mapas multicríticos do círculo, veja as definições no apêndice A.

Lema 2.2.1. Seja $f$ mapa multicrítico e c ponto crítico (non-flat) de grau d. Seja $c \ni W \subseteq S^{1} a$ vizinhança dada na definição 2.2.1. Existe uma vizinhança $U \subseteq W$ tal que:

1. $f$ tem derivada Schwarziana negativa em $U \backslash\{c\}$. Mais precisamente, existe $K=K(c)>0$ tal que para todo $x \in U \backslash\{c\}$ temos:

$$
S f(x)<-\frac{K}{(x-c)^{2}}
$$

2. Existem constantes $0<\alpha<\beta$ tal que para todo $x \in U$

$$
\alpha|x-c|^{d-1}<D f(x)<\beta|x-c|^{d-1} .
$$

Ainda mais, $\alpha$ e $\beta$ podem ser escolhidos tal que $\beta<(3 / 2) \alpha$.

3. Dado um intervalo não vazio $J \subseteq U$ e $x \in J$ temos

$$
D f(x) \leq 3 d \frac{|f(J)|}{|J|} .
$$

4. Dados dois intervalos não vazios $M \subseteq T \subseteq U$ temos:

$$
\mathrm{CrD}(f ; M, T) \leq 9 d^{2} .
$$

Prova do Lema 2.2.1. Da definição de ponto crítico non-flat sabemos que existe uma vizinhança $W$ do ponto crítico $c$ tal que $f(x)=g(\phi(x))+f(c)$ para todo $x \in W$, onde $g$ é o mapa $x \mapsto x^{d}$ e $\phi$ é um difeomorfismo $C^{3} \operatorname{com} \phi(c)=0$. Pela regra da cadeia da derivada temos que $S f=S(g(\phi))(D \phi)^{2}+S \phi$.

Da igualdade $S g(x)=-\frac{\left(d^{2}-1\right)}{2 x^{2}}$, obtemos:

$$
S g(\phi(x))(D \phi(x))^{2}=-\frac{1}{2}(d-1)(d+1)\left(\frac{D \phi(x)}{\phi(x)}\right)^{2} \leq-\frac{A}{(\phi(x))^{2}},
$$

onde $A=\frac{1}{2}(d-1)(d+1) \min _{x}|D \phi(x)|>0$. Em particular:

$$
S f(x)<\frac{-A+S \phi(x)(\phi(x))^{2}}{(\phi(x))^{2}} .
$$


Agora, como $\phi$ é um difeomorfismo, existe $M$ constante positiva tal que $|S \phi(x)|<M$, para todo $x \in W$. Logo, podemos escolher $\delta>0$ tal que $|\phi(x)|<\sqrt{\frac{A}{M}}$, para todo $x \in(c-\delta, c+\delta)=U$. Observe que o fato que $\phi$ seja bi-Lipschitz implica que $|\phi(x)| \asymp|x-c|$ e assim obtemos o Item (1).

O item (2) segue-se do Teorema de Taylor pois:

$$
\lim _{x \rightarrow c}\left(\frac{D f(x)}{|x-c|^{d-1}}\right)=d(D \phi(c))^{d}>0 .
$$

Vamos usar o item (2) para provar o item (3). Seja $J=(a, b) \subseteq U$. Por simetria é suficiente considerar os seguintes dois casos:

1. $c \leq a<b$ : Neste caso para todo $x \in(a, b)$ temos que

$$
\begin{aligned}
\frac{D f(x)|J|}{|f(J)|} & \leq \frac{\beta(x-c)^{d-1}(b-a)}{\alpha \int_{a}^{b}(x-c)^{d-1} d x} \\
& \leq\left(\frac{\beta d}{\alpha}\right) \frac{(b-c)^{d-1}(b-c-a+c)}{(b-c)^{d}-(a-c)^{d}} \\
& =\left(\frac{\beta d}{\alpha}\right)\left(1+\frac{(a-c)^{d}-(b-c)^{d-1}(a-c)}{(b-c)^{d}-(a-c)^{d}}\right) \\
& \leq \frac{\beta d}{\alpha}<\frac{3 d}{2}
\end{aligned}
$$

2. $a<c<b$ : Sem perdida de generalidade podemos assumir que $|a-c|<|c-b|$.

Seja $x \in J$ :

$$
\frac{D f(x)|J|}{|f(J)|} \leq \frac{\beta|x-c|^{d-1}|b-a|}{\int_{c}^{b} D f(t) d t} \leq \frac{2 \beta|b-c|^{d}}{\int_{c}^{b} \alpha(t-c)^{d-1} d t}=\frac{2 \beta d}{\alpha}<3 d .
$$

Finalmente, para provar o Item (4), sejam $L, R$ as duas componentes conexas de $T \backslash M$. Pelo Teorema do Valor Meio existe $z_{0} \in L$ e $z_{1} \in R$ tal que

$$
\mathrm{CrD}(f ; M, T)=\frac{D f\left(z_{0}\right) D f\left(z_{1}\right)|L \cup M||R \cup M|}{|f(L \cup M)||f(R \cup M)|} .
$$

Como $z_{0} \in L \cup M$ e $z_{1} \in R \cup M$ obtemos do Item (3) que:

$$
\mathrm{CrD}(f ; M, T) \leq(3 d)^{2} .
$$

\subsubsection{Propriedades combinatórias}

Como os mapas multicríticos do círculo possuem número de rotação irracional então eles são semiconjugados à rotação correspondente, Teorema A.1.1 no apêndice A. Assim, estes mapas possuem os mesmos tempos de retorno $\left\{q_{n}\right\}_{n \geq 0}$ da rotação rígida correspondente. Isto significa que para cada $n \in \mathbb{N}$ os primeiros $n$ elementos da órbita de $x$ por $f$ estão ordenados do mesmo jeito que os primeiros $n$ elementos da órbita do ponto $(1,0)$ pela correspondente rotação. 
Pelas propriedades aritméticas do número de rotação, para todo $x \in S^{1}$ e para todo $n \in \mathbb{N}$, o intervalo fechado com pontos extremais $x$ e $f^{q_{n}}(x)$, que contém o ponto $f^{q_{n+2}}(x)$ no seu interior, não contém outros iterados $f^{j}(x)$ para $1 \leq j \leq q_{n}-1$. Este intervalo vai ser denotado por $I_{n}(x)$. De fato, escrevemos $I_{n}^{j}(x)=f^{j}\left(I_{n}(x)\right)$ para denotar o $j$-ésimo iterado pela $f$ do intervalo $I_{n}(x)$, para $j \in \mathbb{Z}$.

Para todo $x \in S^{1}$ a coleção de intervalos

$$
\mathcal{P}_{n}(x)=\left\{I_{n}^{i}(x): 0 \leq i \leq q_{n+1}-1\right\} \cup\left\{I_{n+1}^{j}(x): 0 \leq j \leq q_{n}-1\right\}
$$

é uma partição do círculo modulo pontos extremais (veja o apêndice A para uma prova de este fato). Esta partição é chamada a n-ésima partição dinâmica associada ao ponto $x$.

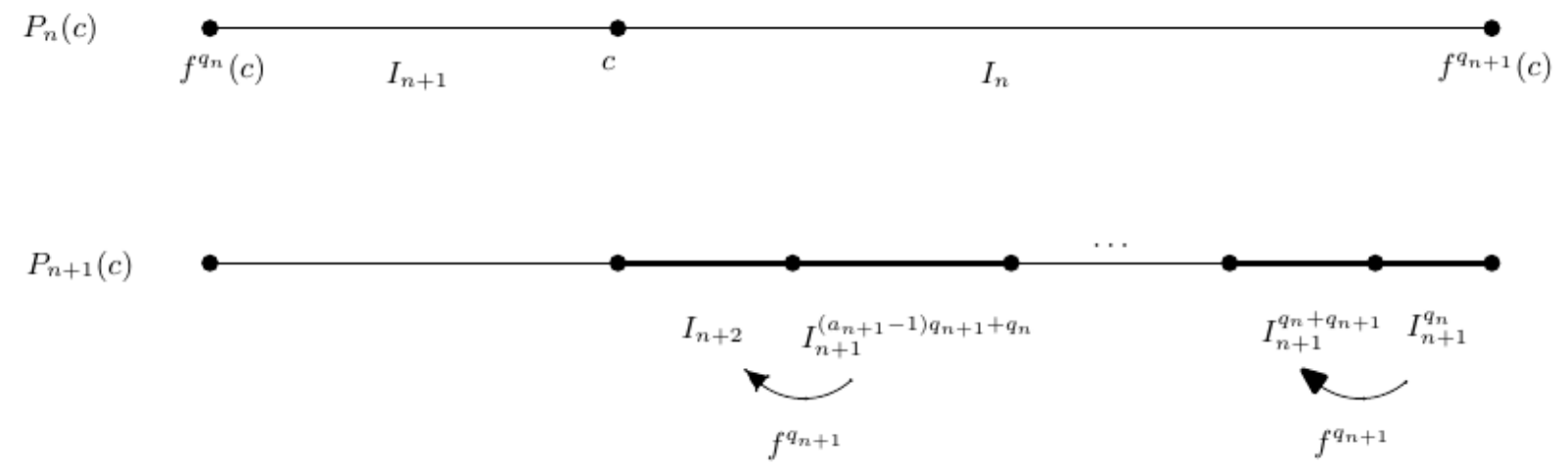

Figura 2.1: Partições dinâmicas nos níveis $n$ e $n+1$.

Para passar da partição $\mathcal{P}_{n}(x)$ para $\mathcal{P}_{n+1}(x)$ observamos o seguinte: o intervalo $I_{n}(x)$ é subdividido nos intervalos $I_{n+1}^{j q_{n+1}+q_{n}}(x)$, para $0 \leq j \leq a_{n+1}$, e no intervalo $I_{n+2}(x)$, porém, o intervalo $I_{n+1}(x)$ fica igual. Portanto, a partição $\mathcal{P}_{n+1}(x)$ é um refinamento (não-estrito) de $\mathcal{P}_{n}(x)$, mas a partição $\mathcal{P}_{n+2}(x)$ forma um refinamento estrito de $\mathcal{P}_{n}(x)$.

Os intervalos de partição $\mathcal{P}_{n}(x)$ são de dois tipos: os intervalos longos, i.e. aqueles intervalos da forma $I_{n}^{i}(x), 0 \leq i<q_{n+1}$, e os intervalos curtos, i.e. aqueles da forma $I_{n+1}^{j}(x)$, para $0 \leq j<q_{n}$. Assim, dois intervalos adjacentes pertencentes à partição dinâmica são ou dois intervalos longos ou um intervalo longo e um curto.

Se o número de rotação é de tipo limitado, digamos por $M>0$, então todo intervalo da partição $\mathcal{P}_{n}(x)$ é subdividido num número limitado (por $M$ ) de intervalos da partição $\mathcal{P}_{n+1}(x)$ ou fica igual. Esta condição sob o número de rotação é importante pois no caso de um único ponto crítico é essencial para melhorar a suavidade da conjugação.

Assim como no caso uni-crítico, a principal consequência da existência da partição dinâmica associada a um ponto crítico é o seguinte resultado:

Teorema 2.2.2 (Real A-priori Bounds). Seja $f$ um mapa multicrítico do círculo com número de rotação irracional e seja c um ponto crítico de $f$. Existe uma constante $C=C(f)>1$ tal que para todo $n \geq 0$ e para todo par de intervalos adjacentes $I, J \in \mathcal{P}_{n}(c)$ temos

$$
\frac{1}{C}|J| \leq|I| \leq C|J| .
$$


Em particular, se $I \in \mathcal{P}_{n}(x)$ e $J \in \mathcal{P}_{n+2}(x)$ é tal que contém o ponto $y \in S^{1}$ então existe $\mu=\mu(f) \in$ $(0,1)$, tal que $|J|<\mu|I|$ para todo $y \in S^{1}$ e para $n \in \mathbb{N}$.

A prova do Teorema 2.2.2 é um pouco longa e depende de alguns lemas auxiliares. Em lugar de seguir as notas originais e inéditas de M. Herman [Her88], ou o trabalho de Petersen [Pet00], vamos imitar a estrategia usada em [dFdM99, Capítulo 3]. Nossa principal motivação para proceder de este jeito, é nosso interesse na geometria das partições dinâmicas $\mathcal{P}_{n}$. Como se vê, a comparabilidade total dada pela desigualdade (2.2.1) não é explícita em [Her88] ou [Pet00]; em vez disso é uma consequência explícita dos argumentos que eles apresentam. Uma motivação adicional é dada pela utilidade de ter uma prova independente. Veja a prova completa de este Teorema no apêndice B.

Observe que o resultado anterior não é válido para rotações rígidas. Se for, teríamos que $\left|I_{n}\right|=$ $a_{n+1}\left|I_{n+1}\right|+\left|I_{n+2}\right|$, e se $a_{n+1}$ é um número arbitrariamente grande então $\left|I_{n}\right|$ é muito maior que $\left|I_{n+1}\right|$, assim a desigualdade (2.2.1) não pode ser válida para nenhuma constante $C>0$.

Para um mapa multicrítico do círculo de classe $C^{3}$ com número de rotação irracional $\rho(f)$ e pontos críticos dados por $\operatorname{Crit}(f)=\left\{c_{0}, c_{1}, \cdots, c_{N-1}\right\}$ definimos o $n$-ésimo scalling ratio associado ao ponto crítico $c_{i}$ como o quociente

$$
s_{n}\left(c_{i}, f\right)=\frac{d\left(f^{q_{n+1}}\left(c_{i}\right), c_{i}\right)}{d\left(f^{q_{n}}\left(c_{i}\right), c_{i}\right)}
$$

onde $d$ denota a distancia usual em $S^{1}$ e $q_{n}$ é o $n$-ésimo tempo de retorno de $f$.

O Teorema 2.2.2 implica que existe uma constante $C=C(f)>0$ tal que cada $n \in \mathbb{N}$ e todo ponto crítico $c_{i}$ de $f$ temos

$$
\frac{1}{C} \leq s_{n}\left(c_{i}, f\right) \leq C
$$

\subsubsection{Propriedades topológicas}

Pelo Teorema 2.2.1 a entropia topológica de todo mapa multicrítico do círculo é zero. De fato, os mapas multicríticos do círculo surgem como bifurcações entre difeomorfismos e endomorfismos do círculo.

O seguinte resultado devido a Swiatek [Swi88] foi usado na prova que o conjunto de parâmetros $a \in[0,1)$, no exemplo 2.1.1, correspondentes a mapas $f_{a}$ com número de rotação irracional, tem medida de Lebesgue zero.

Dada uma família de intervalos $\mathcal{F}$ em $S^{1}$ e um número positivo $m$, dizemos que $\mathcal{F}$ tem multiplicidade de interseção máximo $m$ se todo $x \in S^{1}$ pertence no máximo a $m$ elementos de $\mathcal{F}$.

Lema 2.2.2 (Desigualdade de Cross-ratio). [Swi88] Dado $f: S^{1} \rightarrow S^{1}$ mapa multicrítico do círculo, existe uma constante $C>1$, que depende só de $f$, tal que o seguinte é válido. Seja $\mathcal{I}$ um conjunto finito de indices. Se $M_{i} \Subset T_{i} \subset S^{1}$ são intervalos do círculo tal que a família $\left\{T_{i}: i \in \mathcal{I}\right\}$ tem multiplicidade de interseção no máximo $m$, então

$$
\prod_{i \in \mathcal{I}} \mathrm{CrD}\left(f ; M_{i}, T_{i}\right) \leq C^{m}
$$


Onde $\operatorname{CrD}(f ; M, T)$, é a a distorção do $b$-cross-ratio definido no apêndice A secção A.2, veja também a desigualdade (A.2.1).

Se $f$ é um mapa multicrítico do círculo existem vizinhanças $W_{i}$ de cada ponto crítico (dadas pelos Itens 1 e 2 do Lema 2.2.1),tal que fora de essas vizinhanças o mapa é um difeomorfismo, assim a variação de $\log D f$ em $S^{1} \backslash \cup_{i=0}^{N-1} W_{i}$ é limitada por alguma constante $\rho$ que depende do grau máximo dos pontos críticos. Nestas condições, temos o seguinte resultado

Corolário 2.2.1 (Power-Law). Para todo $x, y \in S^{1}$ com $\left|x-c_{i}\right| \leq\left|y-c_{i}\right|$, temos

$$
\frac{\left|f(x)-f\left(c_{i}\right)\right|}{\left|f(y)-f\left(c_{i}\right)\right|} \leq \gamma\left(\frac{\left|x-c_{i}\right|}{\left|y-c_{i}\right|}\right)^{d_{i}}
$$

onde $\gamma>0$ é uma constante que depende só de $\alpha, \beta, \rho$.

Veja a prova do Corolário 2.2.1 no apêndice A.

\subsubsection{Propriedades ergódicas}

Como todo homeomorfismo do círculo, as rotações rígidas possuem uma medida Boreliana de probabilidade invariante. Lembremos que dada $f: S^{1} \rightarrow S^{1}$, dizemos que $f$ preserva uma medida $\mu$ Boreliana de probabilidade em $S^{1}$ se para todo $B \subseteq S^{1}$ conjunto Boreliano $\mu\left(f^{-1}(B)\right)=\mu(B)$. A conjugação topológica $h$ entre o mapa multicrítico $f$ e a rotação $R_{\rho(f)}$ implica a existência de uma única medida invariante de probabilidade $\mu_{f}$ no círculo que é unicamente ergódica. De fato, esta medida esta definida como $\mu_{f}(A)=\operatorname{Leb}(h(A))$ para todo conjunto Boreliano $A$ de $S^{1}$, onde Leb é a medida de Lebesgue.

Lembremos que todo sistema unicamente ergódico é minimal restrito ao suporte da única medida invariante, neste caso, $\mu_{f}$ (veja por exemplo [VO15]). Além disso, a medida $\mu_{f}$ de um ponto nunca é um número positivo, i.e. não tem átomos. Em contraste, $\mu_{f}$ em conjuntos abertos sempre é um número positivo.

Do Teorema de Yoccoz 2.2.1 obtemos que todo mapa multicrítico do círculo $C^{3}$ com número de rotação irracional é minimal, e assim o suporte de sua única medida invariante de probabilidade $\mu_{f}$ é o círculo inteiro. Em [Kha91, Teorema 4, página 182], Khanin provou que $\mu_{f}$ é singular a respeito da medida de Lebesgue, i.e. existe um conjunto Boreliano $A \subseteq S^{1} \operatorname{com} \mu_{f}(A)=0$ e $\operatorname{Leb}(A)=1$.

O exponente de Lyapunov de um mapa multicrítico do círculo $C^{3}$ sempre é zero:

Teorema 2.2.3. [dFG16] Seja $f: S^{1} \rightarrow S^{1}$ mapa multicrítico do círculo de classe $C^{3}$ com número de rotação irracional, e seja $\mu$ a única medida invariante de probabilidade. Então $\log D f$ pertence ao conjunto $L^{1}(\mu)$ e tem média zero

$$
\int_{S^{1}} \log D f d \mu=0
$$

\subsubsection{Propriedades de regularidade}

Dado que $f$ possui pontos críticos e a rotação correspondente não, é impossível melhorar a regularidade do homeomorfismo que os conjuga. De fato, esta conjugação nunca vai ser absolutamente contínua 
em relação à medida de Lebesgue. Porém, o Teorema 2.2.1 implica que dois mapas multicríticos do círculo com mesmo número de rotação irracional são topologicamente conjugados: dados $f$ e $g$ dois mapas multicríticos do círculo com mesmo número de rotação $\rho$ existem dois homeomorfismos do círculo $h_{f} \mathrm{e}$ $h_{g}$ que satisfazem o seguinte,

$$
h_{f} \circ f=R_{\rho} \circ h_{f} \quad \text { e } \quad h_{f} \circ f=R_{\rho} \circ h_{f}
$$

portanto, o homeomorfismo $h=h_{g}^{-1} \circ h_{f}$ é uma conjugação entre $f$ e $g$, satisfazendo $h \circ f=g \circ h$. Assim, é natural perguntar-se pela regularidade do homeomorfismo $h$. Por exemplo, quais são as condições adicionais para que a conjugação entre dois mapas multicríticos do círculo seja Holder? ou um difeomorfismo?.

No caso uni-crítico, as condições foram achadas. Mas, tanto no caso suave e analítico real o uso do operador renormalização foi essencial. Veja [Avi13, dFdM99, dFdM00, Gua12, GMdM15, GdM13, KT07, KY06]. Dito operador relaciona a rigidez da conjugação direitamente com o número de rotação, mais especificamente, com o crescimento das constantes $a_{n}$ na expansão em fração contínua dada na equação A.1.1.

No caso multicrítico, novos invariantes da conjugação suave surgem.

Seja $f$ é um mapa multicrítico do círculo com número de rotação $\rho(f) \in \mathbb{R} \backslash \mathbb{Q}$ e com $N_{f}$ pontos críticos. Para cada $0 \leq i \leq N_{f}-1$ seja $c_{i}=c_{i}(f)$ o $i$-ésimo ponto crítico de $f$ com criticalidade $d_{i}>1$. Definimos a Assinatura de $f$ como a $\left(2 N_{f}+2\right)$-upla

$$
\left(\rho(f) ; d_{0}, d_{1}, \ldots, d_{N_{f}-1} ; \lambda_{0}, \lambda_{1}, \ldots, \lambda_{N_{f}-1}\right)
$$

onde $\lambda_{i}=\mu_{f}\left[c_{i}, c_{i+1}\right)$ (com a convenção $\left.c_{N_{f}}=c_{0}\right)$.

Sejam $f, g$ dois mapas multicríticos do círculo com mesmo número de pontos críticos e seja $h$ a conjugação entre eles. Se $h$ leva ponto crítico de $f$ em ponto crítico de $g$, dizemos que $h$ é uma conjugação canônica.

Denotamos pelos conjuntos $\operatorname{Crit}(f)=\left\{c_{0}, \cdots, c_{N-1}\right\}$ e $\operatorname{Crit}(g)=\left\{h\left(c_{0}\right), \cdots, h\left(c_{N}\right)\right\}$ os conjuntos formados pelos pontos críticos de $f$ e $g$, respectivamente. Temos a seguinte equivalência,

Lema 2.2.3. h é conjugação canônica se e somente se para todo $0 \leq i \leq N$,

$$
\mu_{f}\left[c_{i}, c_{i+1}\right)=\mu_{g}\left[h\left(c_{i}\right), h\left(c_{i+1}\right)\right] .
$$

Prova do Lema 2.2.3. Seja $h$ uma conjugação canônica entre $f$ e $g$, i.e. se $c_{i}$ é um ponto crítico de $f$ então $h\left(c_{i}\right)$ é um ponto crítico de $g$. Usando a única medida ergódica de $f$ e $g$, e tomando $\left[h\left(c_{i}\right), h\left(c_{i+1}\right)\right]$ como sendo o arco do círculo que não contém mais pontos críticos de $g$ no seu interior, obtemos que

$$
\begin{aligned}
\mu_{g}\left(\left[h\left(c_{i}\right), h\left(c_{i+1}\right)\right]\right) & =\operatorname{Leb}\left(h_{g}\left(\left[h\left(c_{i}\right), h\left(c_{i+1}\right)\right]\right)\right) \\
& =\operatorname{Leb}\left(\left[h_{g}\left(h\left(c_{i}\right)\right), h_{g}\left(h\left(c_{i+1}\right)\right)\right]\right) \\
& =\operatorname{Leb}\left(\left[h_{f}\left(c_{i}\right), h_{f}\left(c_{i+1}\right)\right]\right) \\
& =\operatorname{Leb}\left(h_{f}\left(\left[c_{i}, c_{i+1}\right]\right)\right) \\
& =\mu_{f}\left(\left[c_{i}, c_{i+1}\right]\right) .
\end{aligned}
$$

Reciprocamente, sejam $\mu_{f}$ e $\mu_{g}$ duas medidas de probabilidade invariantes por $f$ e $g$, respectivamente. 
Sejam $h_{f}$ e $h_{g}$ dois mapas no círculo definidos da seguinte forma

$$
\begin{array}{lll}
h_{f}\left(c_{0}\right)=1 & \text { e } & h_{f}(y)=e^{2 \pi i \mu_{f}\left(\left[c_{0}, y\right]\right)}, \\
h_{g}\left(c_{0}^{\prime}\right)=1 & \text { e } & h_{g}(y)=e^{2 \pi i \mu_{g}\left(\left[c_{0}^{\prime}, y\right]\right)},
\end{array}
$$

então $h\left(c_{1}\right)=h_{g}^{-1} \circ h_{f}\left(c_{1}\right)=c_{1}^{\prime}$. Repetindo este procedimento para cada ponto crítico de $f$, obtemos que $h\left(c_{i}\right)=c_{i}^{\prime}$, assim $h$ é uma conjugação canônica.

O seguinte resultado mostra uma condição necessária para que a conjugação canônica entre mapas multicríticos do círculo seja pelo menos $C^{1}$.

Lema 2.2.4. Se h um difeomorfismo de classe $C^{1}$, que é conjugação canônica entre os mapas multicríticos $f$ e $g$. Seja c ponto crítico de $f$, então o grau de c coincide com o grau de $h(c)$.

Prova do Lema 2.2.4. Como $h$ é uma conjugação entre $f$ e $g$ então para cada $x \in S^{1}$,

$$
D f(x)=D h^{-1}(g(h(x))) \circ D g(h(x)) \circ D h(x) .
$$

Suponhamos que os graus dos ponto críticos $c$ e $h(c)$ são $d \geq 3$ e $s>d$, respectivamente. Para $t>0$ temos

$$
\lim _{t \rightarrow 0} \frac{D f(c+t)}{t^{d-1}}=\lim _{t \rightarrow 0} \frac{D h^{-1}(g(h(c+t))) D g(h(c+t)) D h(c+t)}{t^{d-1}} .
$$

Pela definição de ponto crítico non-flat, o lado esquerdo na igualdade previa não é zero, porém, o lado direito é zero. Assim $d=s$.

\subsection{Pares multicríticos comutativos}

Para entender o comportamento da conjugação entre dois mapas multicríticos do círculo, precisamos compreender o comportamento assintótico do operador renormalização de cada mapa multicrítico do círculo. Nesta secção vamos introduzir o espaço onde este operador age, i.e. o espaço formado por mapas do intervalo que são chamados de pares multicríticos comutativos, estes mapas não são mais que mapas crescentes no intervalo.

Observe que o mapa de primeiro retorno da união de intervalos $I_{n+1} \cup I_{n}$ está formado pelos mapas $\left.f^{q_{n}}\right|_{I_{n+1}}$ e $\left.f^{q_{n+1}}\right|_{I_{n}}$, respectivamente.

Definição 2.3.1. Um par multicrítico comutativo de classe $C^{r}$ é um par $\zeta=(\xi, \eta)$ consistindo de dois homeomorfismos $C^{r}$ que preservam orientação $\xi: I_{\xi} \rightarrow \xi\left(I_{\xi}\right)$ e $\eta: I_{\eta} \rightarrow \eta\left(I_{\eta}\right)$ com um número finito de pontos críticos $\gamma_{0}, \cdots, \gamma_{N_{1}}=0$ e $\delta_{0}=0, \delta_{1}, \cdots, \delta_{N_{2}}$, respectivamente, onde o seguinte é satisfeito

1. $I_{\xi}=[\eta(0), 0]$ e $I_{\eta}=[0, \xi(0)]$ são intervalos compactos na reta real;

2. $(\eta \circ \xi)(0)=(\xi \circ \eta)(0) \neq 0$;

3. $D \xi(x)>0$ para todo $\gamma_{i}<x<\gamma_{i+1}, i \in\left\{0,1, \cdots, N_{1}-1\right\}$ e D $\eta(x)>0$ para todo $\delta_{j}<x<\delta_{j+1}-1$, $j \in\left\{0,1, \cdots, N_{2}\right\}$;

4. Todos os pontos críticos são de tipo non-flat com criticalidade impar. A origem é um ponto crítico e tem a mesma criticalidade tanto para $\eta$ como para $\xi$ 
5. Para cada $1 \leq k \leq r$, temos que $D_{-}^{k}(\xi \circ \eta)(0)=D_{+}^{k}(\eta \circ \xi)(0)$, onde $D_{-}$e $D_{+}$representam as $k$-ésimas derivadas a esquerda e à direita, respectivamente.

Todo mapa multicrítico do círculo $f$ de classe $C^{r}, 3 \leq r<\infty$, com número de rotação irracional $\rho(f)$ e com $N$ pontos críticos $c_{0}, \cdots, c_{N}$, induz uma sequência de pares multicríticos comutativos da seguinte forma: primeiro, podemos supor depois de conjugar com um difeomorfismo adequado que $(1,0)$ é um ponto crítico de $f$. Agora, vamos chamar $\widehat{f}$ o levantamento de $f$ correspondente com o cobrimento canônico $t \rightarrow e^{2 \pi i t}$, o qual satisfaz $0<\widehat{f}(0)<1$ e $D \widehat{f}(0)=D \widehat{f}\left(\widehat{c_{1}}\right)=\cdots=D \widehat{f}\left(\widehat{c_{N}}\right)=0$, onde os $\widehat{c}_{i}$ 's são as pre-imagens dos pontos críticos $c_{i}$ pelo cobrimento canônico. Para $n \geq 1$, seja $\widehat{I}_{n}$ o intervalo fechado em $\mathbb{R}$, contendo a origem como um dos seus pontos extremais, o qual é projetado pelo cobrimento canônico em $I_{n}$. Definimos dois mapas $\xi: \widehat{I}_{n+1} \rightarrow \mathbb{R}$ e $\eta: \widehat{I}_{n} \rightarrow \mathbb{R}$ por $\xi=T^{-p_{n}} \circ \widehat{f}^{q_{n}}$ e $\eta=T^{-p_{n+1}} \circ \widehat{f}^{q_{n+1}}$, onde $T$ é a translação à direita por uma unidade na reta real.

Os dois primeiros itens na definição 2.3 .1 são fáceis de verificar. Agora, seja $x \in \widehat{I}_{n}$ então

$$
D \eta(x)=D\left(T^{-p_{n+1}} \circ \widehat{f}^{q_{n+1}}\right)(x)=\prod_{j=0}^{q_{n+1}-1} D \widehat{f}\left(\widehat{f}^{j}(x)\right)=0 \quad \text { see } \quad \widehat{f}^{j}(x) \in\left\{\widehat{c}_{0}, \cdots, \widehat{c}_{N}\right\}
$$

do mesmo jeito para $y \in \widehat{I}_{n+1}$ temos

$$
D \xi(y)=D\left(T^{-p_{n}} \circ \widehat{f}^{q_{n}}\right)(y)=\prod_{i=0}^{q_{n}-1} D \widehat{f}\left(\widehat{f}^{i}(y)\right)=0 \quad \text { see } \widehat{f}^{i}(y) \in\left\{\widehat{c}_{0}, \cdots, \widehat{c}_{N}\right\} .
$$

Pela combinatória, a união dos conjuntos $\left\{I_{n}, \cdots, f^{q_{n+1}-1}\left(I_{n}\right)\right\}$ e $\left\{I_{n+1}, \cdots, f^{q_{n}-1}\left(I_{n+1}\right)\right\}$ cobrem o círculo, então temos que $\operatorname{Crit}\left(\left.\eta\right|_{\widehat{I}_{n}}\right) \cup \operatorname{Crit}\left(\left.\xi\right|_{\widehat{I}_{n+1}}\right)=\left\{\widehat{c}_{0}=0, \widehat{c}_{1}, \cdots, \widehat{c}_{N}\right\}$ e como $\widehat{I}_{n} \cap \widehat{I}_{n+1}=\{0\}$, então $\left.\eta\right|_{\widehat{I}_{n}}$ tem $N_{1}$ pontos críticos e $\left.\xi\right|_{\widehat{I}_{n+1}}$ tem $N-N_{1}=N_{2}$ pontos críticos, todos eles de tipo nonflat com criticalidade impar. As outras propriedades seguem do fato que $\widehat{f}$ é um levantamento de um homeomorfismo e da definição de $\eta$ e $\xi$ ao redor de 0 que é a imagem de algum ponto crítico.

Daqui por diante, vamos denotar por $\left(\left.f^{q_{n}}\right|_{I_{n+1}},\left.f^{q_{n+1}}\right|_{I_{n}}\right)$ o par multicrítico comutativo $\left(\left.\xi\right|_{\widehat{I}_{n+1}},\left.\eta\right|_{\widehat{I}_{n}}\right)$ induzido pelo mapa multicrítico do círculo $f$.

Definimos a altura (ou o período) do par multicrítico comutativo $\zeta=(\eta, \xi)$ e a denotamos por $\chi(\zeta)$, como o número natural $a$ tal que

$$
\eta^{a+1}(\xi(0))<0 \leq \eta^{a}(\xi(0)),
$$

sempre que esse numero natural exista. Em caso contrário, ou seja quando o mapa $\eta$ tenha um ponto fixo, definimos $\chi(\zeta)=\infty$. Observe que a altura do par multicrítico comutativo $\left(\left.f^{q_{n}}\right|_{I_{n+1}},\left.f^{q_{n+1}}\right|_{I_{n}}\right)$ induzido por $f$, é $a_{n+1}$.

Gostaríamos de definir uma distância entre dois pares multicríticos comutativos. Vamos definir antes uma pseudo-métrica. Sejam $\zeta_{1}=\left(\eta_{1}, \xi_{1}\right)$ e $\zeta_{2}=\left(\eta_{2}, \xi_{2}\right)$ dois pares multicríticos comutativos $C^{r}, 3 \leq r<\infty$. Sejam $\tau_{1}:\left[\eta_{1}(0), \xi_{1}(0)\right] \rightarrow[-1,1]$ e $\tau_{2}:\left[\eta_{2}(0), \xi_{2}(0)\right] \rightarrow[-1,1]$ duas transformações Moebius tais que para $i \in\{1,2\}$,

$$
\tau_{i}\left(\eta_{i}(0)\right)=-1, \quad \tau_{i}(0)=0 \text { e } \tau_{i}\left(\xi_{i}(0)\right)=1 .
$$

De fato,

$$
\tau_{1}(t)=\frac{t\left[\eta_{1}(0)-\xi_{1}(0)\right]}{t\left[-\eta_{1}(0)-\xi_{1}(0)\right]+2 \xi_{1}(0) \eta_{1}(0)}
$$


$\mathrm{e}$

$$
\tau_{2}(t)=\frac{t\left[\eta_{2}(0)-\xi_{2}(0)\right]}{t\left[-\eta_{1}(0)-\xi_{2}(0)\right]+2 \xi_{2}(0) \eta_{2}(0)} .
$$

Definição 2.3.2. Para todo $0 \leq r<\infty$ definimos a norma $C^{r}$ entre $\zeta_{1}$ e $\zeta_{2}$ como:

$$
d_{r}\left(\zeta_{1}, \zeta_{2}\right)=\max \left\{\left|\frac{\xi_{1}(0)}{\eta_{1}(0)}-\frac{\xi_{2}(0)}{\eta_{2}(0)}\right|,\left\|\tau_{1} \circ \zeta_{1} \circ \tau_{1}^{-1}-\tau_{2} \circ \zeta_{2} \circ \tau_{2}^{-1}\right\|_{r}\right\}
$$

onde $\|\cdot\|_{r}$ representa a norma $C^{r}$ para mapas no intervalo $[-1,1]$ com uma descontinuidade, no nosso caso, a origem.

Observe que $d_{r}($,$) não é uma métrica, pois apesar de que \|\cdot\|_{r}$ é uma métrica, temos que a quantidade $\left|\frac{\xi_{1}(0)}{\eta_{1}(0)}-\frac{\xi_{2}(0)}{\eta_{2}(0)}\right|$ é invariante por conjugação por homotetias.

\subsection{Renormalização de pares multicríticos comutativos}

Como no caso de mapas unimodais e uni-críticos do círculo, para obter uma conjugação suave entre mapas multicríticos do círculo a ferramenta principal usada é o operador renormalização [dM98]. Este operador age no espaço dos pares multicríticos comutativos com altura finita e o resultado, ao aplicar o operador, é novamente um par multicrítico comutativo. Este procedimento segue, sempre que o par resultante tenha altura finita. Nesta seção vamos introduzir o operador Renormalização, este operador representa o mapa de primeiro retorno de Poincaré numa região. O comportamento assintótico de dito operador vai permitir obter propriedades geométricas dos mapas originais.

Se $\zeta=(\eta, \xi)$ é um par multicrítico comutativo com $\chi(\zeta)=a<\infty$, então o par $\left(\left.\eta\right|_{\left[0, \eta^{a}(\xi(0))\right]},\left.\eta^{a} \circ \xi\right|_{I_{\xi}}\right)$ é, novamente, um par multicrítico comutativo. Portanto, faz sentido introduzir a seguinte definição:

Definição 2.4.1. Seja $\zeta=(\eta, \xi)$ um par multicrítico comutativo com $(\xi \circ \eta)(0) \in I_{\eta}$ e $\chi(\zeta)=a<\infty$. Definimos a pre-renormalização de $\zeta$ como o par multicrítico comutativo

$$
p \mathcal{R}(\zeta)=\left(\left.\eta^{a} \circ \xi\right|_{I_{\xi}},\left.\eta\right|_{\left[0, \eta^{a}(\xi(0))\right]}\right) .
$$

Se $\left(\left.f^{q_{n}}\right|_{I_{n+1}},\left.f^{q_{n+1}}\right|_{I_{n}}\right)$ é um par induzido pelo mapa multicrítico do círculo $f$, então sua prerenormalização é o par $\left(\left.f^{q_{n+2}}\right|_{I_{n+1}},\left.f^{q_{n+1}}\right|_{I_{n+2}}\right)$.

Observação 2.4.1. Para converter a pseudo-métrica numa métrica vamos ter que reescalar o par multicrítico comutativo por um fator linear da seguinte forma: para um par dado $\zeta=(\xi, \eta)$ denotamos $\operatorname{por} \tilde{\zeta}$ o par $\left(\tilde{\xi} \tilde{I}_{\xi},\left.\tilde{\eta}\right|_{\tilde{I}_{\eta}}\right)$ onde $\tilde{\xi}(x)=\xi(x) /\left|I_{\xi}\right|$ para $x \in I_{\xi}$ e $\tilde{\eta}(x)=\eta(x) /\left|I_{\xi}\right|$ para todo $x \in I_{\eta}$. Obtemos, $\tilde{\xi}(0)=\left|I_{\eta}\right| /\left|I_{\xi}\right|=\xi(0) /|\eta(0)|$ e $\tilde{\eta}(0)=-1$. Agora, dados dois pares reescalados $\tilde{\zeta_{1}}$, $\tilde{\zeta}_{2}$ e sejam $\tilde{\tau_{1}}, \tilde{\tau_{2}}$ as transformações Moebius tais que $\tilde{\tau}_{i}\left(\tilde{\eta}_{i}(0)\right)=-1, \tilde{\tau}_{i}(0)=0$ e $\tilde{\tau}_{i}\left(\tilde{\xi}_{i}(0)\right)=1$ para $i \in\{1,2\}$. Então, $\left|\tilde{\zeta_{1}}-\tilde{\zeta_{2}}\right|_{r}=0$ see $\frac{\tilde{\xi_{2}}(0)}{\tilde{\eta}_{2}(0)}=\frac{\tilde{\xi_{1}}(0)}{\tilde{\eta_{1}}(0)}$, isto é, $\frac{\xi_{2}(0)}{\left|\eta_{2}(0)\right|}=\frac{\xi_{1}(0)}{\left|\eta_{1}(0)\right|}$. Entâa, isto implica que $\tilde{\tau_{1}}=\tilde{\tau_{2}}$ o qual significa que

$$
\tilde{\zeta}_{1}={\tilde{\tau_{1}}}^{-1} \circ \tilde{\tau_{2}} \circ \tilde{\zeta_{2}} \circ{\tilde{\tau_{2}}}^{-1} \circ{\tilde{\tau_{1}}}^{=} \tilde{\zeta}_{2} .
$$

Com a observação anterior definimos o operador renormalização:

Definição 2.4.2. O operador renormalização do par multicrítico comutativo $\zeta=(\eta, \xi)$ é o rescalamento ou a normalização de $p \mathcal{R}(\zeta)$ :

$$
\left.\mathcal{R}(\zeta)=\left.\widetilde{\left(\eta^{a} \circ \xi\right.}\right|_{I_{\xi}},\left.\tilde{\eta}\right|_{\left[0, \eta^{a(\xi(0))]}\right.}\right) .
$$


Se $f_{n}=\left(\left.f^{q_{n}}\right|_{I_{n+1}},\left.f^{q_{n+1}}\right|_{I_{n}}\right)$ é induzido por um mapa multicrítico do círculo $f$ então sua renormalização, denotada por $\mathcal{R}\left(f_{n}\right)$, esta definida como o par

$$
\mathcal{R}\left(f_{n}\right)=\left(\left.B_{n+1} \circ f^{q_{n+2}} \circ B_{n+1}^{-1}\right|_{[-1,0]},\left.B_{n+1} \circ f^{q_{n+1}} \circ B_{n+1}^{-1}\right|_{\left[0, \frac{\left|I_{n+2}\right|}{\left|I_{n+1}\right|}\right]}\right),
$$

onde $B_{n+1}: I_{n+1} \cup I_{n+2} \rightarrow\left[-1, \frac{\left|I_{n+2}\right|}{\left|I_{n+1}\right|}\right]$ é a única transformação de Moebius que leva $f^{q_{n+1}}\left(c_{0}\right) \mathrm{em}-1$, $c_{0}$ em 0 e o ponto $f^{q_{n+2}}\left(c_{0}\right)$ em $\frac{\left|I_{n+2}\right|}{\left|I_{n+1}\right|}$. Observe que neste caso $\mathcal{R}\left(f_{n}\right)$ coincide com $\mathcal{R}^{n+1}(f)$ onde $f$ é o $\operatorname{par}\left(\left.f\right|_{I_{1}},\left.f^{a_{0}}\right|_{I_{0}}\right)$.

Por simplicidade da notação, escrevemos $\tilde{f}^{q_{n+i}}=B_{n+1} \circ f^{q_{n+i}} \circ B_{n+1}^{-1}$ para $i=1,2$, e $\lambda_{n+1}(f)=$ $\left|I_{n+2}\right| /\left|I_{n+1}\right|$, assim

$$
\mathcal{R}^{n+1}(f)=\left(\left.\tilde{f}^{q_{n+2}}\right|_{[-1,0]},\left.\tilde{f}^{q_{n+1}}\right|_{\left[0, \lambda_{n+1}(f)\right]}\right) .
$$

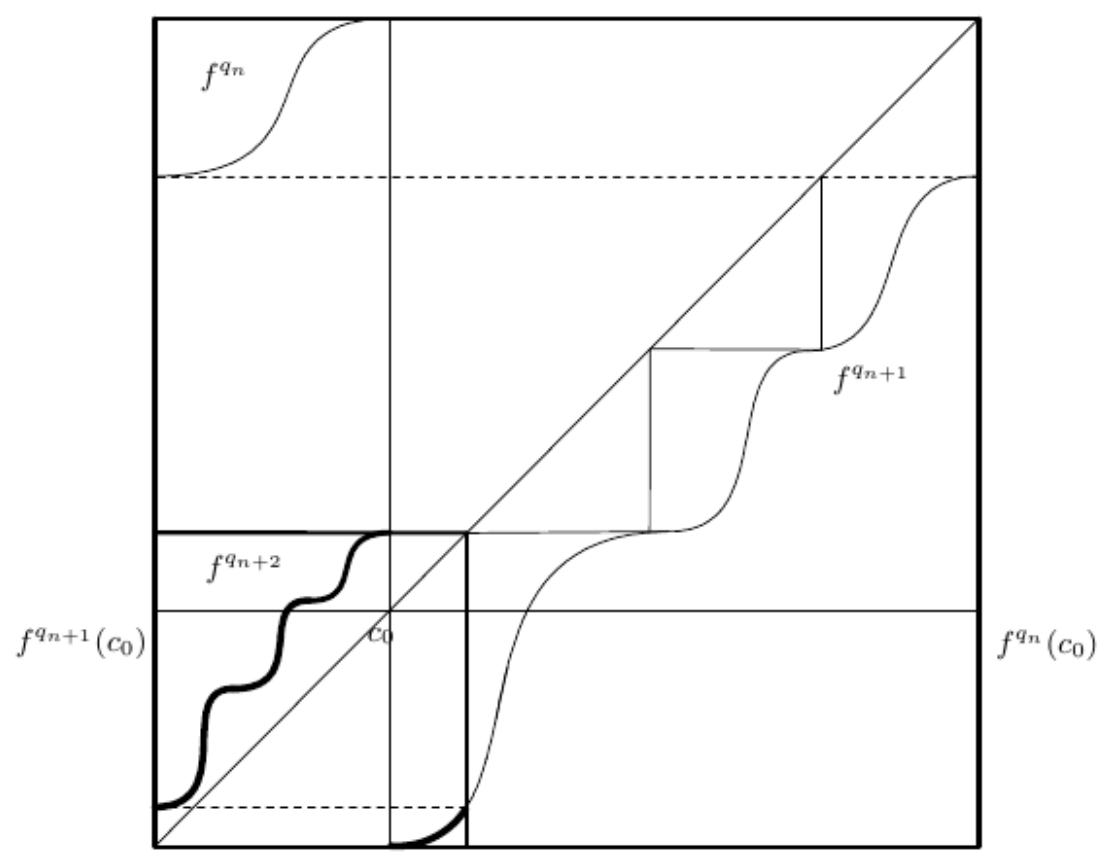

Figura 2.2: A n-ésima e $n+1$-ésima renormalização de $f$.

Com a notação previa, se

$$
\mathcal{R}^{n+1}(f)=\left(\left.\tilde{f}^{q_{n+2}}\right|_{[-1,0]},\left.\tilde{f}^{q_{n+1}}\right|_{\left[0, \lambda_{n+1}(f)\right]}\right) \quad \text { e } \quad \mathcal{R}^{n+1}(g)=\left(\left.\tilde{g}^{q_{n+2}}\right|_{[-1,0]},\left.\tilde{g}^{q_{n+1}}\right|_{\left[0, \lambda_{n+1}(g)\right]}\right)
$$

são duas renormalizações então a distância $C^{r}$ entre elas é

$$
\begin{aligned}
& d_{1}\left(\mathcal{R}^{n+1}(f), \mathcal{R}^{n+1}(g)\right)= \\
& \quad \max \left\{\left|\lambda_{n+1}(f)-\lambda_{n+1}(g)\right|,\left\|\tau_{n+1, f} \circ \mathcal{R}^{n+1}(f) \circ \tau_{n+1, f}^{-1}-\tau_{n+1, g} \circ \mathcal{R}^{n+1}(g) \circ \tau_{n+1, g}^{-1}\right\|_{r}\right\},
\end{aligned}
$$

onde $\tau_{n+1, f}$ e $\tau_{n+1, g}$ são duas transformações Moebius que fixam os pontos $-1,0$ e tais que

$$
\tau_{n+1, f}\left(\lambda_{n+1}(f)\right)=1=\tau_{n+1, g}\left(\lambda_{n+1}(g)\right)=1 .
$$


Como o operador renormalização age em particular no espaço dos pares multicríticos comutativos, então cada vez que apliquemos o operador e o par obtido tenha altura finita podemos aplicar este operador novamente. Mais precisamente, se $\zeta$ é um par multicrítico comutativo com $\chi\left(\mathcal{R}^{j}(\zeta)\right)<\infty$ para $0 \leq j \leq n-1$, dizemos que $\zeta$ é n-vezes renormalizável, e se $\chi\left(\mathcal{R}^{j}(\zeta)\right)<\infty$ para todo $j \in \mathbb{N}$ dizemos que $\zeta$ é infinitamente renormalizável. No último caso, definimos o número de rotação do par multicrítico comutativo $\zeta$ como sendo o número $\rho(\zeta)=\theta$, cuja expansão em fração contínua esta dada por

$$
\left[\chi(\zeta), \chi(\mathcal{R}(\zeta)), \cdots, \chi\left(\mathcal{R}^{n}(\zeta)\right), \cdots\right]
$$

Se $\zeta$ é um par induzido por um mapa multicrítico do círculo $f$ com número de rotação irracional $\rho(f)$, então $\zeta$ é infinitamente renormalizável e sue número de rotação coincide com $\rho(f)$. Em particular, o operador renormalização age como um shift à esquerda na expansão de fração contínua de $\rho(f)$ : se $\rho(\zeta)=\left[a_{0}, a_{1}, \cdots\right]$ então $\rho\left(\mathcal{R}^{n}(\zeta)\right)=\left[a_{n}, a_{n+1}, \cdots\right]$. 


\section{Capítulo 3}

\section{Rigidez quase-simétrica de mapas multicríticos do círculo}

Neste capítulo provamos uma das consequências do Teorema das Real Bounds: dados dos mapas multicríticos do círculo com mesmo número de rotação irracional e mesmo número de pontos críticos, então a conjugação canônica é quase-simétrica (veja a definição no Apêndice A). A constante de quasesimetria depende unicamente da constante do Teorema das Real Bounds, teorema 2.2.2.

Mais precisamente, o Teorema principal deste capítulo é o seguinte:

Teorema 3.0.1 (Conjugação quase-simétrica). Sejam $f, g: S^{1} \rightarrow S^{1}$ mapas multicríticos do círculo de classe $C^{r}(r \geq 3)$ com mesmo número de rotação irracional e o mesmo número de pontos críticos de tipo non-flat, e seja $h: S^{1} \rightarrow S^{1}$ o homeomorfismo canônico que conjuga $f$ com $g$, então $h$ é um mapa quase-simétrico.

Como consequência temos o seguinte resultado

Corolário 3.0.1. Se h é o homeomorfismo canônico que conjuga dois mapas multicríticos do círculo de classe $C^{3}$ com mesmo número de rotação irracional e mesmo número de pontos críticos de tipo non-flat, então h é $\alpha$-Holder para $\alpha>0$.

Veja uma prova deste corolário no apêndice C, Lema C.0.1.

Lembramos que no caso uni-crítico o Teorema 3.0.1 é válido, i.e. dados dois mapas criticos do círculo com mesmo número de rotação irracional existe um homeomorfismo quase-simétrico que leva ponto crítico em ponto crítico. A prova de este resultado se encontra em [dFdM99], onde os autores provam muito mais que isso. Em contraste, se os mapas são um mapa crítico do círculo e a correspondente rotação rígida, então eles não podem ser quase-simetricamente conjugados, veja [dMvS93].

A prova do Teorema 3.0.1 que apresentamos aqui é a mesma encontrada no artigo [EdF15] que, em sua vez, foi inspirada na prova no caso uni-crítico em [dFdM99]. Esta última esta baseada num critério devido a Carleson [Car67]. Para enunciar-lo corretamente vamos precisar dar a seguinte definição:

Definição 3.0.3. Uma fine grid é uma sequência $\left\{\mathcal{Q}_{n}\right\}_{n \geq 0}$ de partições finitas do círculo que satisfazem as seguintes condiçôes.

(a) Cada partição $\mathcal{Q}_{n+1}$ é um refinamento estrito de $\mathcal{Q}_{n}$; 
(b) Existe $a \geq 2$, número natural, tal que cada intervalo $\Delta \in \mathcal{Q}_{n}$ é união disjunta de máximo a intervalos de $\mathcal{Q}_{n+1}$;

(c) Existe uma constante $\rho>1$ tal que $\rho^{-1}|\Delta| \leq\left|\Delta^{\prime}\right| \leq \rho|\Delta|$ para todo par de intervalos adjacentes $\Delta, \Delta^{\prime} \in \mathcal{Q}_{n}$.

Os números a, $\rho$ são chamados de constantes da fine grid.

Notemos a seguinte consequência da definição anterior: Existem constantes $0<\alpha<\beta<1$ dependendo só das constantes da fine grid a, $\rho$ tal que, sempre que $\Delta \in \mathcal{Q}_{n}, \Delta^{*} \in \mathcal{Q}_{n-1}$ e $\Delta \subset \Delta^{*}$, temos

$$
\alpha\left|\Delta^{*}\right| \leq|\Delta| \leq \beta\left|\Delta^{*}\right|
$$

De fato, podemos tomar $\alpha=\left(a \rho^{a-1}\right)^{-1}$ e $\beta=\left(1+\rho^{-1}\right)^{-1}$.

Observação 3.0.2. Se o número de rotação do mapa multicrítico $f$ é de tipo limitado, i.e. $\left|a_{n}\right| \leq M$ para algum $M \in \mathbb{N}$, então as partiçôes dinâmicas associadas ao ponto crítico c formam uma fine grid $\operatorname{com} a=M$.

Com a definição da fine grid, estamos em condições para dar o critério de Carleson,

Proposição 3.0.1. Seja $\left\{\mathcal{Q}_{n}\right\}_{n \geq 0}$ uma fine grid em $S^{1}$, e sejam a, $\rho$ as constantes da fine grid. Seja $h: S^{1} \rightarrow S^{1}$ um homeomorfismo tal que

$$
\left|\frac{\left|\Delta^{\prime}\right|}{\left|\Delta^{\prime \prime}\right|}-\frac{\left|h\left(\Delta^{\prime}\right)\right|}{\left|h\left(\Delta^{\prime \prime}\right)\right|}\right| \leq \lambda
$$

para todo par de intervalos adjacentes $\Delta^{\prime}, \Delta^{\prime \prime} \in \mathcal{Q}_{n}$, para todo $n \geq 0$, onde $\lambda>0$ é uma constante dada. Então existe uma constante $K=K(a, \rho, \lambda)>1$ tal que $h$ é quase-simétrica com constante de quase-simetria igual a $\mathrm{K}$.

Veja o Apêndice §C para uma prova da Proposição 3.0.1.

Pela observação 3.0.2, o teorema 2.2.2 e o critério 3.0.1 dois mapas multicríticos do círculo com mesmo número de rotação irracional de tipo limitado são conjugados por um mapa quase simétrico. Assim, só temos que estudar o caso número de rotação não limitado. Usando o critério 3.0.1 só temos que construir uma fine grid tal que todo par de átomos adjacentes satisfazem a desigualdade 3.0.2.

No que segue, vamos construir uma fine grid que cumpra com as hipóteses do critério 3.0.1.

Ressaltamos que a imagem de uma fine grid por um homeomorfismo que satisfaz a condição (3.0.2) é também uma fine grid. Reciprocamente, se um homeomorfismo $h$ é um isomorfismo entre duas fine grids, i.e. se $h$ estabelece uma correspondência entre seus intervalos, então $h$ deve satisfazer a condição $(3.0 .2)$.

\subsection{Geometria das partições dinâmicas}

Nesta seção vamos apresentar as consequências geométricas do Teorema das Real Bounds (2.2.2) que serão necessárias para provar a Proposição 3.0.1 para o caso número de rotação irracional arbitrário. A partir de agora, $f$ representa um mapa multicrítico do círculo onde o Teorema 2.2.2 é válido. 


\subsubsection{Intervalos que se intersectam são comparáveis}

Nosso primeiro resultado afirma que dois intervalos de partições dinâmicas associadas a dois pontos críticos distintos no mesmo nível cuja interseção é diferente de vazio, são comparáveis.

Lema 3.1.1. Sejam $c, c^{\prime}$ dois pontos críticos de f. Se $\Delta \in \mathcal{P}_{n}(c)$ e $\Delta^{\prime} \in \mathcal{P}_{n}\left(c^{\prime}\right)$ são dois intervalos tal que $\Delta \cap \Delta^{\prime} \neq \varnothing$, então $|\Delta| \asymp\left|\Delta^{\prime}\right|$, i.e. eles são comparáveis.

Demonstração. Seja $C=C(f)>1$ a constante dada pelo Teorema das real bounds (Teorema 2.2.2). Para $\Delta$ e $\Delta^{\prime}$ existem três casos a considerar segundo os tipos de intervalos: longo/longo, longo/curto, e curto/curto.

(i) Temos $\Delta=I_{n}^{i}(c)$ e $\Delta^{\prime}=I_{n}^{j}\left(c^{\prime}\right)$, onde $0 \leq i, j<q_{n+1}$. Aqui, podemos assumir que $f^{j}\left(c^{\prime}\right) \in$ $\Delta=\left[f^{i}(c), f^{i+q_{n}}(c)\right]$. Então $f^{i+q_{n}}(c) \in \Delta^{\prime}=\left[f^{j}\left(c^{\prime}\right), f^{j+q_{n}}\left(c^{\prime}\right)\right]$, e assim estamos na situação descrita na Figura 3.4(a). Usando a monotonicidade de $f^{q_{n}}$, podemos ver que $\Delta^{\prime} \subset \Delta \cup f^{q_{n}}(\Delta)$. Aplicando o Lema B.1.3 em $x=f^{i+q_{n}}(c)$, temos que $\Delta=\left[f^{-q_{n}}(x), x\right]$ e $f^{q_{n}}(\Delta)=\left[x, f^{q_{n}}(x)\right]$ satisfazem $\left|f^{q_{n}}(\Delta)\right| \leq C|\Delta|$, e de isto segue-se que $\left|\Delta^{\prime}\right| \leq(1+C)|\Delta|$. Reciprocamente, temos que $\Delta \subset f^{-q_{n}}\left(\Delta^{\prime}\right) \cup \Delta^{\prime}$. Mais uma vez aplicando o Lema B.1.3, esta vez em $x=f^{j}\left(c^{\prime}\right)$, deduzimos como antes que $\left|f^{-q_{n}}\left(\Delta^{\prime}\right)\right| \leq C\left|\Delta^{\prime}\right|$, e portanto $|\Delta| \leq(1+C)\left|\Delta^{\prime}\right|$. Consequentemente $\Delta$ e $\Delta^{\prime}$ são comparáveis neste caso.

(ii) Agora $\Delta=I_{n}^{i}(c)$ e $\Delta^{\prime}=I_{n+1}^{j}\left(c^{\prime}\right)$, onde $0 \leq i<q_{n+1}$ e $0 \leq j<q_{n}$. Aqui, consideramos o intervalo $I_{n+1}^{i+q_{n}}(c) \subset \Delta$. Este intervalo compartilha um ponto extremal com $\Delta$ (a saber $f^{i+q_{n}}(c)$ ) e dito intervalo é um intervalo de $\mathcal{P}_{n+1}(c)$. Em particular, $\left|I_{n+1}^{i+q_{n}}(c)\right| \asymp|\Delta|$, pelo Teorema das real bounds. Existem dois sub-casos. Se $\Delta^{\prime} \cap I_{n+1}^{i+q_{n}}(c) \neq \varnothing$, então, como $\Delta^{\prime}$ também pertence a $\mathcal{P}_{n+1}\left(c^{\prime}\right)$, o caso (i) acima afirma que $\left|\Delta^{\prime}\right| \asymp\left|I_{n+1}^{i+q_{n}}(c)\right|$, e portanto $\Delta^{\prime}$ é comparável com $\Delta$ neste caso. Por outro lado, se $\Delta^{\prime} \cap I_{n+1}^{i+q_{n}}(c)=\varnothing$, então devemos ter $f^{j}\left(c^{\prime}\right) \in \Delta$ (veja a Figura 3.4(b)). Neste caso, consideramos o intervalo $I_{n}^{j}\left(c^{\prime}\right) \in \mathcal{P}_{n}\left(c^{\prime}\right)$, o qual tem $f^{j}\left(c^{\prime}\right)$ como um do seus pontos extremais. Então temos $\Delta \cap I_{n}^{j}\left(c^{\prime}\right) \neq \varnothing$, e novamente pelo caso (i) podemos dizer que $|\Delta| \asymp\left|I_{n}^{j}\left(c^{\prime}\right)\right|$. Mas pelo Teorema das real bounds temos $\left|I_{n}^{j}\left(c^{\prime}\right)\right| \asymp\left|I_{n+1}^{j}\left(c^{\prime}\right)\right|=\left|\Delta^{\prime}\right|, \operatorname{logo} \Delta^{\prime}$ é comparável com $\Delta$ também neste caso.

(iii) Temos $\Delta=I_{n+1}^{i}(c)$ e $\Delta^{\prime}=I_{n+1}^{j}\left(c^{\prime}\right)$, onde $0 \leq i, j<q_{n}$. Este caso é inteiramente análogo com o caso (i).

\subsubsection{Os intervalos críticos são longos}

Vamos considerar o mapa de primeiro retorno do intervalo $I_{n}\left(c_{0}\right) \cup I_{n+1}\left(c_{0}\right)$, ou equivalentemente o par de mapas $\left.f^{q_{n}}\right|_{I_{n+1}\left(c_{0}\right)},\left.f^{q_{n+1}}\right|_{I_{n}\left(c_{0}\right)}$. Note que o mapa de primeiro retorno em torno do ponto $c_{0}$ (que é o ponto crítico para os dois mapas no par), tem máximo $N-1$ pontos críticos: alguns deles no intervalo $I_{n}\left(c_{0}\right)$, e os outros em $I_{n+1}\left(c_{0}\right)$. O seguinte resultado afirma que os intervalos da partição dinâmica do nível $n+1$ (ou seja, $\mathcal{P}_{n+1}\left(c_{0}\right)$ ) que contém estos pontos críticos no mapa de primeiro retorno na partição de nível $n$, são comparáveis com o intervalo parente $I_{n}\left(c_{0}\right)$ ou $I_{n+1}\left(c_{0}\right)$.

Lema 3.1.2. Seja $0 \leq k<a_{n+1}$ tal que o intervalo $f^{q_{n}+k q_{n+1}}\left(I_{n+1}\left(c_{0}\right)\right) \subset I_{n}\left(c_{0}\right)$ contém um ponto crítico do mapa $f^{q_{n+1}}$. Entâao

$$
\left|f^{q_{n}+k q_{n+1}}\left(I_{n+1}\left(c_{0}\right)\right)\right| \asymp\left|I_{n}\left(c_{0}\right)\right| .
$$


Demonstração. Se $k=0$ não temos nada a provar, pois pelas real bounds já sabemos que $\left|f^{q_{n}}\left(I_{n+1}\left(c_{0}\right)\right)\right| \asymp$ $\left|I_{n}\left(c_{0}\right)\right|$. Logo vamos assumir que $1 \leq k \leq a_{n+1}-1$. Vamos denotar $\Delta=f^{q_{n}+k q_{n+1}}\left(I_{n+1}\left(c_{0}\right)\right)$ nesta prova. Seja $0<j \leq q_{n+1}$ que satisfaz $f^{j}(\Delta) \ni c_{1}$, onde $c_{1} \neq c_{0}$ é outro ponto crítico de $f$. Observemos que $I_{n}^{j}\left(c_{0}\right)=f^{j}\left(I_{n}\left(c_{0}\right)\right) \supset f^{j}(\Delta)$. Afirmamos que $\left|f^{j}(\Delta)\right| \asymp\left|f^{j}\left(I_{n}\left(c_{0}\right)\right)\right|$. Esto é uma consequência dos seguintes dois fatos.

a)

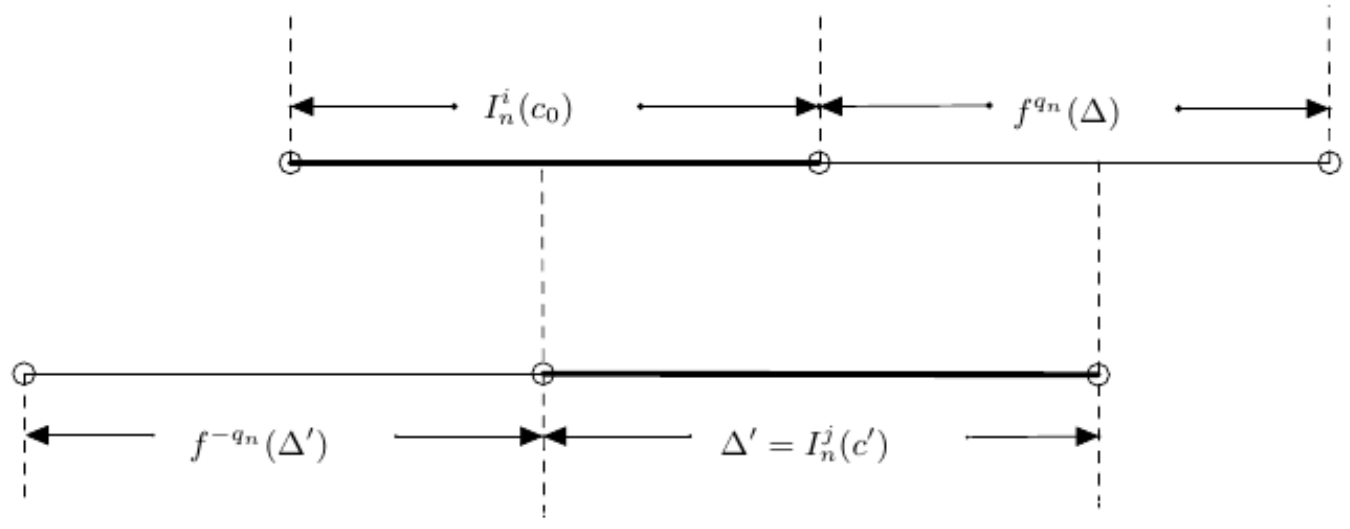

b)

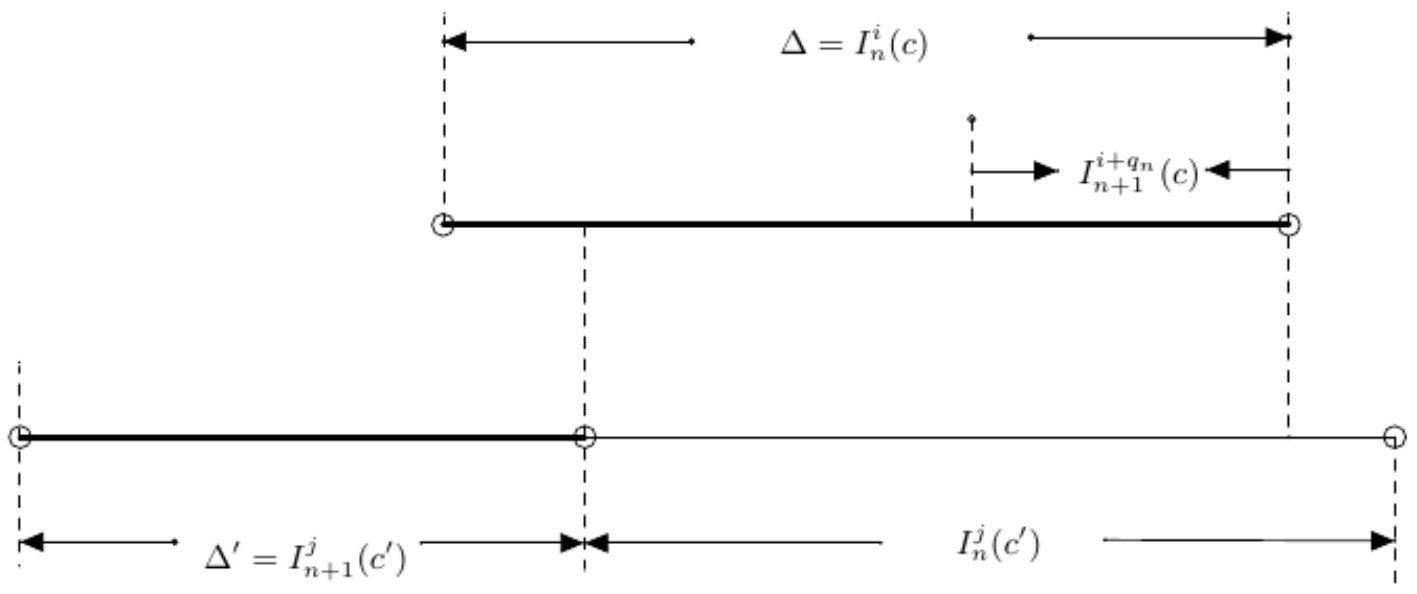

Figura 3.1: Os casos longo/longo e longo/curto do Lema 3.1.1.

(i) Temos $\left|I_{n}^{j}\left(c_{0}\right)\right| \asymp\left|I_{n+1}\left(c_{1}\right)\right|$. De fato, estes dois intervalos tem interseção não vazia (eles contém o ponto $\left.c_{1}\right)$, e como $I_{n}^{j}\left(c_{0}\right) \in \mathcal{P}_{n}\left(c_{0}\right)$ e $I_{n+1}\left(c_{1}\right) \in \mathcal{P}_{n}\left(c_{1}\right)$, então sua comparabilidade segue-se do Lema 3.1.1.

(ii) Por outro lado, temos $\left|I_{n+1}\left(c_{1}\right)\right| \asymp\left|f^{j}(\Delta)\right|$. Isto é verdade pois primeiro temos que

$$
j+q_{n}+k q_{n+1} \leq q_{n}+(k+1) q_{n+1} \leq q_{n}+a_{n+1} q_{n+1}=q_{n+2},
$$


consequentemente

$$
f^{j}(\Delta)=I_{n+1}^{j+q_{n}+k q_{n+1}}\left(c_{0}\right) \in \mathcal{P}_{n+1}\left(c_{0}\right) .
$$

Como $I_{n+1}\left(c_{1}\right) \in \mathcal{P}_{n+1}\left(c_{1}\right)$, e $f^{j}(\Delta) \cap I_{n+1}\left(c_{1}\right) \supset\left\{c_{1}\right\} \neq \varnothing$, podemos aplicar novamente o Lema 3.1 .1 e podemos concluir que $I_{n+1}\left(c_{1}\right)$ e $f^{j}(\Delta)$ são comparáveis.

Com isto, procedemos da seguinte forma. Consideremos o fecho do espaço entre $\Delta$ e $I_{n+1}^{q_{n}}$ no interior de $I_{n}\left(c_{0}\right)$, a saber o intervalo $J=\bigcup_{i=1}^{k-1} I_{n+1}^{q_{n}+i q_{n+1}}\left(c_{0}\right)$. Notemos que se $k=1$ então $J=\emptyset$; neste caso $\Delta$ e $I_{n+1}^{q_{n}}$ são intervalos adjacentes de $\mathcal{P}_{n+1}\left(c_{0}\right)$, aqui eles são comparáveis pelas real bounds (Teorema 2.2.2) e não temos nada a provar. Portanto vamos assumir que $k \geq 2$, tal que $J \neq \varnothing$. Já sabemos da afirmação anterior que $\left|f^{j}(\Delta)\right| \asymp\left|I_{n}^{j}\left(c_{0}\right)\right|$, e as real bounds dizem ainda que $\left|I_{n}^{j}\left(c_{0}\right)\right| \asymp\left|I_{n+1}^{j+q_{n}}\left(c_{0}\right)\right|$. Além disso, temos que $I_{n+1}^{j+q_{n}+q_{n+1}}\left(c_{0}\right) \subseteq f^{j}(J) \subset I_{n}^{j}\left(c_{0}\right)$. Como $\left|I_{n+1}^{j+q_{n}+q_{n+1}}\left(c_{0}\right)\right| \asymp\left|I_{n+1}^{j+q_{n}}\left(c_{0}\right)\right|$, pois eles são intervalos consecutivos de $\mathcal{P}_{n+1}\left(c_{0}\right)$, segue-se que $\left|f^{j}(J)\right| \asymp\left|I_{n+1}^{j+q_{n}}\left(c_{0}\right)\right|$. Em outras palavras, os intervalos consecutivos $f^{j}(\Delta), f^{j}(J)$ e $I_{n+1}^{j+q_{n}}\left(c_{0}\right)$ são dois a dois comparáveis. Em particular, o $b-c r o s s$ ratio determinado por estes três intervalos é limitado por acima e por baixo, i.e. existe uma constante $K>1$ dependendo só da constante $C$ das real bounds tal que

$$
K^{-1} \leq b\left(f^{j}(J), f^{j}(T)\right) \leq K
$$

Escrevemos $T=\Delta \cup J \cup I_{n+1}^{q_{n}}\left(c_{0}\right)$. Notemos que $T, f(T), \ldots, f^{j}(T)$ são dois a dois disjuntos. Portanto, pela Desigualdade de Cross-Ratio aplicado no homeomorfismo $f^{j}($ e $m=1)$, temos $D\left(f^{j} ; J, T\right) \leq C$, ou equivalentemente $b\left(f^{j}(J), f^{j}(T)\right) \leq C b(J, T)$. Usando a estimativa por baixo na desigualdade (3.1.2), temos que $b(J, T) \geq C^{-1} K^{-1}$, isto é,

$$
\frac{|\Delta|\left|I_{n+1}^{q_{n}}\left(c_{0}\right)\right|}{|\Delta \cup J|\left|J \cup I_{n+1}^{q_{n}}\left(c_{0}\right)\right|} \geq(C K)^{-1} .
$$

Mas, como $J \supseteq I_{n+1}^{q_{n}+q_{n+1}}\left(c_{0}\right)$, e os intervalos $I_{n+1}^{q_{n}+q_{n+1}}\left(c_{0}\right)$ e $I_{n+1}^{q_{n}}\left(c_{0}\right)$ são intervalos adjacentes de $\mathcal{P}_{n+1}\left(c_{0}\right)$, temos pelas real bounds

$$
|\Delta \cup J|>|J| \geq\left|I_{n+1}^{q_{n}+q_{n+1}}\left(c_{0}\right)\right| \geq C^{-1}\left|I_{n+1}^{q_{n}}\left(c_{0}\right)\right| .
$$

Além disso, $\left|I_{n+1}^{q_{n}}\left(c_{0}\right)\right| \geq C^{-1}\left|I_{n}\left(c_{0}\right)\right|$, mais uma vez pelas real bounds. Colocando todo isto na desigualdade (3.1.3), deduzimos que

$$
|\Delta| \geq C^{-2} K^{-1}\left|J \cup I_{n+1}^{q_{n}}\left(c_{0}\right)\right|>C^{-3} K^{-1}\left|I_{n}\left(c_{0}\right)\right| .
$$

Isto mostra que $\Delta$ e $I_{n}\left(c_{0}\right)$ são comparáveis. Assim (3.1.1) fica estabelecida, e a prova do Lema 3.1.2 esta completa.

\subsubsection{Uma partição auxiliar}

Nesta subseção vamos construir um refinamento adequado da partição dinâmica $\mathcal{P}_{n}\left(c_{0}\right)$ (para cada $n \geq 1)$. Esta nova partição, que vai ser denotada por $\mathcal{P}_{n}^{*}\left(c_{0}\right)$, é mais fina que $\mathcal{P}_{n}\left(c_{0}\right)$ mas mais grossa que $\mathcal{P}_{n+1}\left(c_{0}\right)$. A criação de esta partição vai ser necessária na construção da fine grid na seção §3.2.

De aqui em frente, para $0 \leq k<a_{n+1}$, denotamos por $\Delta_{k}$ o intervalo $f^{q_{n}+k q_{n+1}}\left(I_{n+1}\left(c_{0}\right)\right)$. Note que cada intervalo $\Delta_{k}$ é um átomo da partição dinâmica $\mathcal{P}_{n+1}\left(c_{0}\right)$, e que

$$
\bigcup_{k=0}^{a_{n+1}-1} \Delta_{k}=I_{n}\left(c_{0}\right) \backslash I_{n+2}\left(c_{0}\right) .
$$


Consideramos os tempos $0 \leq k_{1}<k_{2}<\cdots<k_{r}<a_{n+1}$ tal que $\Delta_{k_{i}}$ contém um ponto critico de $f^{q_{n+1}}$. Estes tempos vão ser chamados de critical times no nível $n$. Para facilidade na notação, definimos $k_{0}=0$. Note que $f^{q_{n+1}}$ tem máximo $N$ pontos críticos no intervalo $I_{n}\left(c_{0}\right)$, onde $N$ é o número total de pontos críticos de $f$. Como cada ponto crítico pertence no máximo a dois dos intervalos $\Delta_{k}$ 's, temos que $r \leq 2 N$. Assim, embora que o inteiro não-negativo $r$ pode depender de $n$ (o nível da renormalização), seu valor varia ao longo apenas de um número finito de valores. Os critical times $k_{i}$ também dependem de $n$. Os intervalos $\Delta_{k_{i}}$ para $0 \leq i \leq r$ vão ser chamados de sítios criticos.

Para cada $i=0,1, \ldots, r-1$, seja $G_{i} \subseteq I_{n}\left(c_{0}\right) \backslash I_{n+2}\left(c_{0}\right)$ o espaço entre dois sítios críticos consecutivos $\Delta_{k_{i}}$ e $\Delta_{k_{i+1}}$ em $I_{n}\left(c_{0}\right)$, a saber o intervalo

$$
G_{i}=\bigcup_{k=k_{i}+1}^{k_{i+1}-1} \Delta_{k}
$$

Também definimos, para $i=r$,

$$
G_{r}=\bigcup_{k=k_{r}+1}^{a_{n+1}-1} \Delta_{k}
$$

O intervalo $G_{i}$ vai ser chamado de $i$-ésimo ponte de $I_{n}\left(c_{0}\right)$. Veja a figura 3.2. Observamos que pode acontecer que $G_{i}=\varnothing$ para algum ou para todos os valores de $i$.

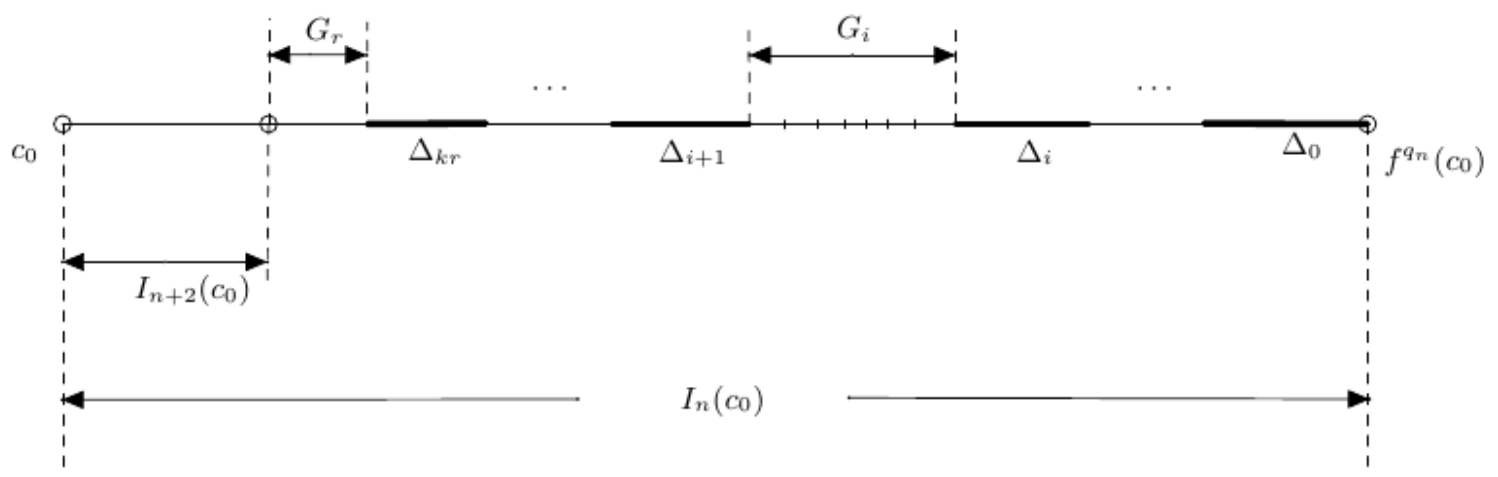

Figura 3.2: Pontes e sítios críticos.

Lema 3.1.3. Todo ponte não-vazio $G_{i}$ é comparável com $I_{n}\left(c_{0}\right)$.

Demonstração. Se $G_{i} \neq \varnothing$, então $G_{i}$ contém pelo menos o intervalo $\Delta_{k_{i}+1}$, adjacente ao $\Delta_{k_{i}}$, e assim temos $\left|G_{i}\right| \geq\left|\Delta_{k_{i}+1}\right| \asymp\left|\Delta_{k_{i}}\right|$, pelas real bounds. Pelo Lema 3.1.2, obtemos $\left|\Delta_{k_{i}}\right| \asymp\left|I_{n}\left(c_{0}\right)\right|$. Como além disso $G_{i} \subset I_{n}\left(c_{0}\right)$, segue-se que $\left|G_{i}\right| \asymp\left|I_{n}\left(c_{0}\right)\right|$.

Assim, temos a seguinte decomposição de $I_{n}\left(c_{0}\right) \backslash I_{n+2}\left(c_{0}\right)$ como a união de no máximo $2 r+2 \leq$ $4 N+2$ intervalos:

$$
I_{n}\left(c_{0}\right) \backslash I_{n+2}\left(c_{0}\right)=\bigcup_{i=0}^{r} \Delta_{k_{i}} \cup \bigcup_{i=0}^{r} G_{i} .
$$

Pelos Lemas 3.1.2 e 3.1.3, como das real bounds, cada intervalo da decomposição anterior é comparável com $I_{n}\left(c_{0}\right)$. Em particular, eles são todos comparáveis dois a dois. 
Observação 3.1.1. Notemos que a imagem de cada sítio crítico $\Delta_{k_{i}}$ pelo mapa $f^{q_{n+1}}$ também é comparável com $I_{n}\left(c_{0}\right)$ : isto é verdade pois $f^{q_{n+1}}\left(\Delta_{k_{i}}\right)=\Delta_{k_{i}+1}$ é adjacente a $\Delta_{k_{i}}$ em $\mathcal{P}_{n+1}\left(c_{0}\right)$. Do mesmo modo, a imagem de cada ponte $G_{i}$ por $f^{q_{n+1}}$ também é comparável a $I_{n}\left(c_{0}\right)$, pois vamos ter que $i<r$ e $f^{q_{n+1}}\left(G_{i}\right)$ contém o sítio crítico $\Delta_{k_{i+1}}$, ou $i=r$, em cujo caso $f^{q_{n+1}}\left(G_{r}\right)$ contém $I_{n+2}\left(c_{0}\right)$.

Agora vamos mapear a decomposição (3.1.4) para frente pela $f$ para obter a decomposição correspondente dos intervalos longos $I_{n}^{j}\left(c_{0}\right) \in \mathcal{P}_{n}\left(c_{0}\right)$, para $j=1,2, \ldots, q_{n+1}-1$. Deste jeito obtemos uma partição nova do círculo $\mathcal{P}_{n}^{*}\left(c_{0}\right)$ (modulo pontos extremais). Mais precisamente, seja

$$
\begin{aligned}
\mathcal{P}_{n}^{*}\left(c_{0}\right)= & \left\{f^{j}\left(\Delta_{k_{i}}\right): 0 \leq i \leq r ; 0 \leq j \leq q_{n+1}-1\right\} \\
& \cup\left\{f^{j}\left(G_{i}\right): 0 \leq i \leq r ; 0 \leq j \leq q_{n+1}-1\right\} \\
& \cup\left\{f^{j}\left(I_{n+2}\right): 0 \leq j \leq q_{n+1}-1\right\} \\
& \cup\left\{f^{\ell}\left(I_{n+1}\right): 0 \leq \ell \leq q_{n}-1\right\} .
\end{aligned}
$$

Esta partição refina $\mathcal{P}_{n}\left(c_{0}\right)$, mas não estritamente pois cada intervalo curto de $\mathcal{P}_{n}\left(c_{0}\right)$ fica invariável pelo procedimento prévio. Generalizando a nomenclatura anterior, todos os intervalos de $\mathcal{P}_{n}^{*}\left(c_{0}\right)$ da forma $f^{j}\left(\Delta_{k_{i}}\right)$ são chamados sítios críticos, e aqueles da forma $f^{j}\left(G_{i}\right)$ são chamados de pontes.

Proposição 3.1.1. Dois intervalos consecutivos de $\mathcal{P}_{n}^{*}\left(c_{0}\right)$ são comparáveis.

Demonstração. Pelo Teorema das real bounds (Teorema 2.2.2), a partição $\mathcal{P}_{n}\left(c_{0}\right)$ tem a propriedade de comparabilidade requerida. Logo é suficiente provar que todos os pontes e todos sítio críticos de $\mathcal{P}_{n}^{*}\left(c_{0}\right)$ dentro de cada intervalo longo $I_{n}^{j}\left(c_{0}\right) \in \mathcal{P}_{n}\left(c_{0}\right)$ são comparáveis a $I_{n}^{j}\left(c_{0}\right)$. Nós já sabemos isso para $j=0$ (veja Lema 3.1.3 e o parágrafo que segue da prova). Para os outros valores de $j$, mapeamos $I_{n}^{j}\left(c_{0}\right)$ para frente por $f^{q_{n+1}-j}$ sob $I_{n}^{q_{n+1}}\left(c_{0}\right) \subset I_{n}\left(c_{0}\right) \cup I_{n+1}\left(c_{0}\right)$ e aplicamos a Desigualdade Cross-Ratio 2.2.2, junto com a Observação 3.1.1.

\subsubsection{Mapas quase-parabólicos}

Para construir a fine grid na seção $3.2 \mathrm{em}$ baixo, vamos precisar não somente todos os intervalos de muitas partições $\mathcal{P}_{n}^{*}\left(c_{0}\right)$, mas também vamos ter que quebrar ainda mais alguns de tais intervalos, de uma forma adequada. Nesta subseção e na próxima, vamos mostrar como quebrar tais intervalos do jeito adequado.

Antes de tudo, vamos lembrar a definição de mapa quase-parabólico [dFdM99, §4.1].

Definição 3.1.1. Um mapa quase-parabólico é um difeomorfismo de classe $C^{3}$

$$
\phi: J_{1} \cup J_{2} \cup \cdots \cup J_{\ell} \rightarrow J_{2} \cup J_{3} \cup \cdots \cup J_{\ell+1},
$$

onde $J_{1}, J_{2}, \ldots, J_{\ell+1}$ são intervalos consecutivos do círculo (ou da reta), com as seguintes propriedades.

(i) $\phi\left(J_{\nu}\right)=J_{\nu+1}$ para todo $1 \leq \nu \leq \ell$;

(ii) A derivada Schwarziana de $\phi$ é negativa em toda parte.

O inteiro positivo $\ell$ é chamado o comprimento de $\phi$, e o número positivo real

$$
\sigma=\min \left\{\frac{\left|J_{1}\right|}{\left|\cup_{\nu=1}^{\ell} J_{\nu}\right|}, \frac{\left|J_{\ell}\right|}{\left|\cup_{\nu=1}^{\ell} J_{\nu}\right|}\right\}
$$


é chamado a largura de $\phi$.

Notemos que a dinâmica de um mapa quase-parabólico $\phi$ é mais ou menos como uma translação, e cada intervalo $J_{\nu}$ é um domínio fundamental para $\phi$ (modulo pontos extremais). A geometria dos domínios fundamentais está representada no seguinte lema, devido a Yoccoz.

Lema 3.1.4 (Yoccoz). Seja $\phi: \bigcup_{\nu=1}^{\ell} J_{\nu} \rightarrow \bigcup_{\nu=2}^{\ell+1} J_{\nu}$ um mapa quase-parabólico com comprimento $\ell$ e largura $\sigma$. Existe uma constante $C_{\sigma}>1$ (que depende de $\sigma$ mas não de $\ell$ ) tal que, para todo $\nu=1,2, \ldots, \ell$, temos

$$
\frac{C_{\sigma}^{-1}|I|}{[\min \{\nu, \ell+1-\nu\}]^{2}} \leq\left|J_{\nu}\right| \leq \frac{C_{\sigma}|I|}{[\min \{\nu, \ell+1-\nu\}]^{2}}
$$

onde $I=\bigcup_{\nu=1}^{\ell} J_{\nu}$ é o dominio do mapa $\phi$.

Uma prova deste resultado pode ser encontrada em [dFdM99, Apêndice B].

Se definimos a ordem do domínio fundamental $J_{\nu} \operatorname{como} \operatorname{ord}\left(J_{\nu}\right)=\min \{\nu, \ell+1-\nu\}$, então o lema anterior afirma que o comprimento de um domínio fundamental de todo mapa quase-parabólico é inversamente proporcional com o quadrado de sua ordem.

O seguinte lema mostra uma maneira de juntar os domínios fundamentais de um mapa quaseparabólico.

Lema 3.1.5. Seja $\phi$ um mapa quase-parabólico com $\ell \geq 4$. Seja $I=\bigcup_{\nu=1}^{\ell} J_{\nu}$ o seu domínio, e seja $d \in \mathbb{N}$ o maior natural tal que $2^{d+1} \leq \ell / 2$. Existe uma cadeia descendente de intervalos fechados (veja a figura 3.3)

$$
I=M_{0} \supset M_{1} \supset \cdots \supset M_{d+1}
$$

para os quais se satisfaz o seguinte: Se $L_{i}, R_{i}$ denotam as componentes conexas à esquerda e à direita, respectivamente, de $M_{i} \backslash M_{i+1}$ para todo $0 \leq i \leq d$, então as seguintes propriedades são válidas:

(i) Cada intervalo $L_{i}, R_{i}$ é a união de exatamente $2^{i}$ intervalos consecutivos, os quais são domínios fundamentais de $I$.

(ii) Temos

$$
I=\bigcup_{i=0}^{d} L_{i} \cup M_{d+1} \cup \bigcup_{i=0}^{d} R_{i}
$$

(iii) Para todo $0 \leq i \leq d$ temos $\left|L_{i}\right| \asymp\left|M_{i+1}\right| \asymp\left|R_{i}\right|$, com constantes de comparabilidade dependendo unicamente da largura $\sigma$ de $\phi$.

Demonstração. Para cada $0 \leq i \leq d$, definimos

$$
L_{i}=\bigcup_{\nu=2^{i}}^{2^{i+1}-1} J_{\nu} ; R_{i}=\bigcup_{\nu=\ell+2-2^{i+1}}^{\ell+1-2^{i}} J_{\nu}
$$




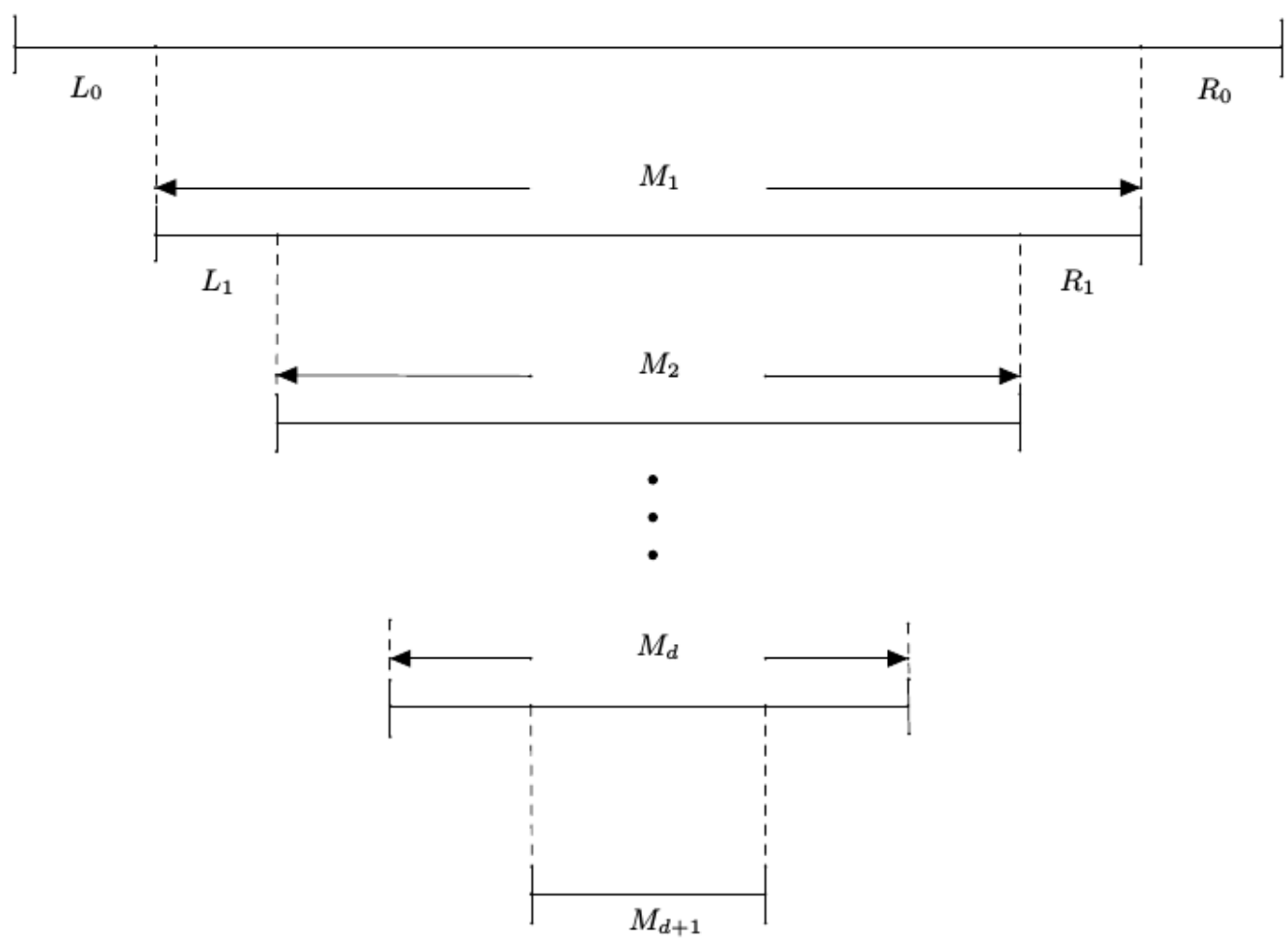

Figura 3.3: Decomposição balanceada do dominio de um mapa quase-parabólico.

Além disso, para todo $1 \leq i \leq d+1$, seja

$$
M_{i}=\bigcup_{\nu=2^{i}}^{\ell+1-2^{i}} J_{\nu}
$$

Então os itens (i) e (ii) seguem-se imediatamente. Assim, só temos que provar o item (iii). Fixemos $0 \leq i \leq d$. Em tudo o que se segue, as constantes de comparabilidade são universais ou dependem unicamente da constante $C_{\sigma}$ do Lema de Yoccoz 3.1.4. Aplicando aquele lema, obtemos que

$$
\left|L_{i}\right|=\sum_{\nu=2^{i}}^{2^{i+1}-1}\left|J_{\nu}\right| \asymp\left(\sum_{\nu=2^{i}}^{2^{i+1}-1} \frac{1}{\nu^{2}}\right)|I| \asymp 2^{-i}|I| .
$$

E também,

$$
\left|R_{i}\right| \asymp 2^{-i}|I|
$$

Aliás, podemos escrever

$$
\left|M_{i+1}\right|=\sum_{\nu=2^{i+1}}^{2^{i+2}-1}\left|J_{\nu}\right| \asymp 2\left(\sum_{2^{i+1} \leq \nu \leq \frac{\ell}{2}} \frac{1}{\nu^{2}}\right)|I|=2 A|I|,
$$

onde o número $A$ satisfaz

$$
\sum_{\nu=2^{i+1}}^{2^{i+2}-1} \frac{1}{\nu^{2}} \leq A \leq \sum_{\nu=2^{i+1}}^{\infty} \frac{1}{\nu^{2}}
$$


Como a função $1 / \nu^{2}$ diminui monotonamente, então a soma $\sum_{\nu=2^{i+1}}^{\infty} \frac{1}{\nu^{2}}$ é comparável com a integral $\int_{2^{i+1}}^{\infty} 1 / x^{2}$. Dado que

$$
\lim _{b \rightarrow \infty} \int_{2^{i+1}}^{b} \frac{1}{x^{2}} d x=\lim _{b \rightarrow \infty}-\frac{1}{b}+\frac{1}{2^{i+1}}=\frac{1}{2^{i+1}},
$$

então a soma $\sum_{\nu=2^{i+1}}^{\infty} \frac{1}{\nu^{2}}$ é comparável com $2^{-i-1}$.

Portanto, as duas somas que aparecem em (3.1.11) são comparáveis a $2^{-i-1}$. Consequentemente, (3.1.10) e (3.1.11) implicam que

$$
\left|M_{i+1}\right| \asymp 2^{-i}|I|
$$

Combinando (3.1.8), (3.1.9) e (3.1.12), obtemos o item (iii), que era o que queríamos provar.

Observação 3.1.2. Dado um intervalo I particionado nos intervalos $J_{\nu}, 1 \leq \nu \leq \ell$, como acima, uma decomposição da forma (3.1.7) satisfaz as propriedades (i), (ii), (iii) do Lema 3.1 .5 é chamada de decomposição balanceada de I (em relação ao seu determinada partição em átomos). Assim, Lema 3.1.5 pode ser reescrita afirmando que o domínio de um mapa quase-parabólico sempre admite uma decomposição balanceada. Nessa decomposição, os intervalos $M_{i}, 0 \leq i \leq d+1$, são chamados intervalos centrais, enquanto que os intervalos $L_{i}, R_{i}, 0 \leq i \leq d$, são chamados laterais. O inteiro positivo $d$ é a profundeza da decomposição.

A seguir, enunciamos um resultado relacionado com a comparabilidade de uniões de imagens de intervalos por um mapa quase-parabólico. Este fato sera útil na seção §3.2.1.

Proposição 3.1.2. Seja $\phi$ um mapa quase-parabólico com domínios fundamentais $J_{\nu}(1 \leq \nu \leq \ell)$, para $\ell \geq 4$. Para todo $1 \leq k<l<m \leq \ell$, é válido

$$
\frac{\left|J_{l+1}\right|+\left|J_{l+2}\right|+\cdots+\left|J_{m}\right|}{\left|J_{k+1}\right|+\left|J_{k+2}\right|+\cdots+\left|J_{l}\right|} \asymp \frac{k(m-l)}{m(l-k)},
$$

com constante de comparabilidade dependendo só da largura $\sigma$ de $\phi$.

Prova da Proposição 3.1.2. Temos duas opções, $\min \{l, \ell+1-l\}=l$ ou $\min \{l, \ell+1-l\}=\ell+1-l$. Suponhamos $\min \{l, \ell+1-l\}=l$, o outro caso é análogo.

A hipóteses implica o seguinte,

$$
\left|J_{k+1}\right|+\left|J_{k+2}\right|+\cdots+\left|J_{l}\right| \asymp|I| \frac{(l-k-1)}{l(k+1)} .
$$

Isto é verdadeiro pois, pelo Lema de Yoccoz, para todo $i \in\{k+1, \cdots, l\}$ temos $\left|J_{i}\right| \asymp \frac{|I|}{i^{2}}$, onde $I=J_{1} \cup J_{2} \cup \cdots \cup J_{\ell}$. Portanto, usando o critério da integral

$$
\sum_{i=k+1}^{l}\left|J_{i}\right| \asymp \sum_{i=k+1}^{l} \frac{|I|}{i^{2}} \asymp|I| \frac{(l-k-1)}{l(k+1)} .
$$

Agora, para limitar a soma $\left|J_{l+1}\right|+\left|J_{l+2}\right|+\cdots+\left|J_{m}\right|$ temos duas opções: 
1. Se $\min \{m, l+1-m\}=m$. Então Lema 3.1 .4 implica que o comprimento de cada intervalo $J_{j}$ para $l+1 \leq j \leq m$, é comparável com $|I| / j^{2}$, então pelo critério da integral, obtemos

$$
\left|J_{l+1}\right|+\left|J_{l+2}\right|+\cdots+\left|J_{m}\right| \asymp|I| \frac{(m-l-1)}{m(l+1)} .
$$

Portanto,

$$
\frac{\left|J_{l+1}\right|+\left|J_{l+2}\right|+\cdots+\left|J_{m}\right|}{\left|J_{k+1}\right|+\left|J_{k+2}\right|+\cdots+\left|J_{l}\right|} \asymp \frac{l(m-l-1)(k+1)}{m(l+1)(l-k-1)} \asymp \frac{l(m-l)(k+1)}{m(l-k)(l+1)} \asymp \frac{k(m-l)}{m(l-k)} .
$$

2. Se $\min \{m, l+1-m\}=l+1-m$. O comprimento de cada intervalos $J_{j}$ para $l+1 \leq j \leq(\ell / 2)$ é comparável com $|I| / j^{2}$ e o comprimento de cada intervalo $J_{j}$ para $\ell / 2+1 \leq j \leq m$ é comparável com $|I| /(l+1-j)^{2}$, isto pelo Lema de Yoccoz. Usando mais uma vez o critério da integral obtemos,

$$
\left|J_{l+1}\right|+\left|J_{l+2}\right|+\cdots+\left|J_{\ell / 2}\right| \asymp|I| \frac{(\ell-2(l+1))}{\ell(l+1)},
$$

$\mathrm{e}$

$$
\left|J_{(\ell / 2)+1}\right|+\left|J_{(\ell / 2)+2}\right|+\cdots+\left|J_{m}\right| \asymp|I| \frac{(2 m-l-2)}{m(l+2)} .
$$

Portanto,

$$
\left|J_{l+1}\right|+\left|J_{l+2}\right|+\cdots+\left|J_{m}\right| \asymp|I| \frac{\left[\left(\ell^{2}+2 \ell\right)(m-l-1)-4 m(l+1)\right]}{\ell(l+1) m(l+2)} .
$$

Consequentemente, como $\ell \geq 4$,

$$
\frac{\left|J_{l+1}\right|+\left|J_{l+2}\right|+\cdots+\left|J_{m}\right|}{\left|J_{k+1}\right|+\left|J_{k+2}\right|+\cdots+\left|J_{l}\right|} \asymp \frac{l(k+1)\left(\ell^{2}+2 \ell\right)(m-l-1)}{m(\ell+2) \ell(l+1)(l-k-1)} \asymp \frac{l(m-l-1)(k+1)}{m(l-k-1)(l+1)} \asymp \frac{k(m-l)}{m(l-k)} .
$$

Observação 3.1.3. Sejam $I, I^{*}$ dois intervalos fechados com $I^{*}$ contido no interior de $I$. Seja $I^{*}$ dividido num número finito $\ell$ de intervalos, consecutivos $J_{\nu}, 1 \leq \nu \leq \ell$ como acima, e suponha que estes intervalos satisfazem as desigualdades (3.1.6) (para alguma constante $C_{\sigma}$ ) - logo temos uma decomposição balanceada de $I^{*}$ (como no Lema 3.1.5). Então, adicionando componentes laterais de $I \backslash I^{*}$ na coleção de $J_{\nu}$ 's e re-etiquetando estes $\ell+2$ intervalos desde o primeiro até o último, temos que as desigualdades (3.1.6) seguem sendo válidas para a nova coleção (com uma constante diferente de comparabilidade) Assim, obtemos uma decomposição balanceada de I. Esta observaçâa vai ser usada na prova do Corolário 3.1.1.

\subsubsection{Decomposição balanceada de pontes}

Vamos classificar os intervalos da partição $\mathcal{P}_{n}^{*}\left(c_{0}\right)$ em duas classes:

(a) Intervalos regulais: São todos os intervalos curtos de $\mathcal{P}_{n}\left(c_{0}\right)$, os quais pertencem a $\mathcal{P}_{n}^{*}\left(c_{0}\right)$, todos os intervalos da forma $f^{j}\left(I_{n+2}\right)$ (para $0 \leq j \leq q_{n+1}-1$ ), todos os sítio críticos $f^{j}\left(\Delta_{k_{i}}\right)$ (com $\left.0 \leq i \leq r, 0 \leq j \leq q_{n+1}-1\right)$, junto com todos aqueles pontes $G_{i, j}=f^{j}\left(G_{i}\right)$ que tem menos de 1,000 intervalos de $\mathcal{P}_{n+1}\left(c_{0}\right)$ neles (i.e., aqueles com $\left.k_{i+1}-k_{i} \leq 1,000\right)$.

(b) Intervalos Sela-node: São os restantes pontes; a saber, os $G_{i, j}$ cuja decomposição como união de intervalos de $\mathcal{P}_{n+1}\left(c_{0}\right)$ tem pelo menos 1,000 de tais intervalos nele (i.e., aqueles com $k_{i+1}-k_{i}>$ 
$1,000)$.

Procedendo analogamente como em [dFdM99, §4.3], vamos mostrar com ajuda do Lema de Yoccoz 3.1.4 como obter uma decomposição balanceada de um ponte sela-node. Antes de tudo, vamos assumir o seguinte fato. Conjugando nosso mapa multicrítico do círculo $f$ com um difeomorfismo $C^{3}$ adequado, podemos assumir sem perda de generalidade que o mapa $f$ é canônico, no sentido que cada ponto critico $c_{k}$ tem uma vizinhança $\mathcal{U}_{k} \subseteq S^{1}$ tal que para todo $x \in \mathcal{U}_{k}$

$$
f(x)=f\left(c_{k}\right)+\left(x-c_{k}\right)\left|x-c_{k}\right|^{s_{k}-1}
$$

onde $s_{k}>1$ é a power-law de $c_{k}$ (como no apêndice A). Isto implica que a derivada Schwarziana $S f$ é negativa em cada $\mathcal{U}_{k}$, i.e., para todo $x \in \mathcal{U}_{k} \backslash\left\{c_{k}\right\}$, temos

$$
S f(x)=-\frac{s_{k}^{2}-1}{2\left(x-c_{k}\right)^{2}}<0
$$

Escrevemos $\mathcal{U}=\bigcup_{k=0}^{N-1} \mathcal{U}_{k}$, e tomamos $\mathcal{V} \subset S^{1}$ um conjunto aberto que não contém nenhum dos pontos críticos de $f$ mas é tal que $\mathcal{U} \cup \mathcal{V}=S^{1}$.

Agora, consideremos o ponte não vazio $G_{i} \subset I_{n}\left(c_{0}\right)$, ou seja

$$
G_{i}=\bigcup_{k=k_{i}+1}^{k_{i+1}-1} \Delta_{k}
$$

Definimos o ponte reduzido $G_{i}^{*}$ associado a $G_{i}$ como

$$
G_{i}^{*}=\bigcup_{k=k_{i}+2}^{k_{i+1}-2} \Delta_{k}
$$

Em outras palavras, $G_{i}^{*}$ é o intervalo $G_{i}$ sem seus dois intervalos laterais. Em particular, se $G_{i}$ esta formado por menos de dois intervalos de $\mathcal{P}_{n+1}\left(c_{0}\right)$, então $G_{i}^{*}=\varnothing$. Com esta terminologia estabelecemos o seguinte resultado.

Lema 3.1.6. Existem $n_{0}=n_{0}(f) \in \mathcal{N}$ tal que o seguinte é válido para todo $n \geq n_{0}$. Para cada ponte reduzido não vazio $G_{i}^{*} \subset I_{n}\left(c_{0}\right)$, o mapa $\left.f^{q_{n+1}}\right|_{G_{i}^{*}}$ tem derivada Schwarziana negativa em todo ponto, i.e., para todo $x \in G_{i}^{*}$ temos $S f^{q_{n+1}}(x)<0$.

Demonstração. Dado $x \in G_{i}^{*}$ e $n \in \mathbb{N}$, pela regra da cadeia da derivada Schwarziana temos

$$
S f^{q_{n+1}}(x)=\sum_{j=0}^{q_{n+1}-1} S f\left(f^{j}(x)\right)\left[D f^{j}(x)\right]^{2} .
$$

Notemos que a soma do lado direito de (3.1.14) pode ser dividida como duas somas $\Sigma_{1}^{(n)}(x)$ e $\Sigma_{2}^{(n)}(x)$ onde

$$
\Sigma_{1}^{(n)}(x)=\sum_{j: I_{n}^{j}\left(c_{0}\right) \subset \mathcal{U}} S f\left(f^{j}(x)\right)\left[D f^{j}(x)\right]^{2},
$$

e $\Sigma_{2}^{(n)}(x)$ é a soma sob os termos restantes. 
Seja $\delta_{n}=\max _{0 \leq j<q_{n+1}}\left|I_{n}^{j}\left(c_{0}\right)\right|$. Sabemos que $\delta_{n} \rightarrow 0$ quando $n \rightarrow \infty$, pois $f$ é topologicamente conjugado à rotação. Assim, escolhendo $n_{1}=n_{1}(f)$ suficientemente grande tal que $\delta_{n}$ seja menor que o número de Lebesgue do cobrimento $\{\mathcal{U}, \mathcal{V}\}$ do círculo, para todo $n \geq n_{1}$. Então obtemos, para todo $n \geq n_{1}$ e todo $x \in G_{i}^{*}$,

$$
\left|\Sigma_{2}^{(n)}(x)\right| \leq \sum_{j: I_{n}^{j}\left(c_{0}\right) \subset \mathcal{V}}\left|S f\left(f^{j}(x)\right)\right|\left[D f^{j}(x)\right]^{2},
$$

Agora vamos proceder através dos passos seguintes.

(i) Como $I_{n}\left(c_{0}\right) \subset \mathcal{U}$, a soma do lado direito de (3.1.15) inclui o termo com $j=0$, a saber $S f(x)$, e por (3.1.13) temos

$$
S f(x)=-\frac{s_{0}^{2}-1}{2\left(x-c_{0}\right)^{2}} .
$$

Todos os outros termos em (3.1.15) também são negativos. Como $\left|x-c_{0}\right| \asymp\left|I_{n}\left(c_{0}\right)\right|$ (veja (ii) abaixo), obtemos de (3.1.15) e de (3.1.17) que

$$
\Sigma_{1}^{(n)}(x)<-\frac{K_{1}}{\left|I_{n}\left(c_{0}\right)\right|^{2}},
$$

onde $K_{1}>0$ é uma constante que depende só das real bounds e do expoente da power-law $s_{0}$.

(ii) Como não há pontos críticos de $f^{q_{n+1}} \operatorname{em} \operatorname{int}\left(G_{i}\right) \supset G_{i}^{*}$, o mapa $f^{q_{n+1}}: \operatorname{int}\left(G_{i}\right) \rightarrow f^{q_{n+1}}\left(\operatorname{int}\left(G_{i}\right)\right)$ é um difeomorfismo ${ }^{1}$. O mesmo pode ser dito dos mapas $f^{j}: \operatorname{int}\left(G_{i}\right) \rightarrow f^{j}\left(\operatorname{int}\left(G_{i}\right)\right)$ para $0 \leq$ $j \leq q_{n+1}-1$. Pelas real bounds e a Proposição 3.1.1, temos que $\left|f^{j}\left(G_{i}^{*}\right)\right| \asymp\left|f^{j}\left(G_{i}\right)\right| \asymp\left|I_{n}^{j}\left(c_{0}\right)\right|$ para todo $0 \leq j \leq q_{n+1}-1$. Aliás, as duas componentes de $G_{i} \backslash G_{i}^{*}$ são comparáveis com $G_{i}^{*}$ (e também com $I_{n}\left(c_{0}\right)$ ). Portanto, pelo principio de distorção de Koebe (Lema A.2.1) e o Teorema do valor meio, temos que

$$
\left|D f^{j}(x)\right| \asymp \frac{\left|f^{j}\left(G_{i}^{*}\right)\right|}{\left|G_{i}^{*}\right|} \asymp \frac{\left|I_{n}^{j}\left(c_{0}\right)\right|}{\left|I_{n}\left(c_{0}\right)\right|},
$$

para todo $x \in G_{i}^{*}$ e para todo $0 \leq j \leq q_{n+1}-1$.

(iii) Uma vez tendo provado o item (ii), estamos prontos para estimar o lado direito de (3.1.16). Usando (3.1.19), concluímos que existe uma constante $K_{2}>0$ dependendo só das real bounds tal que

$$
\begin{aligned}
\left|\Sigma_{2}^{(n)}(x)\right| & \leq K_{2} \sum_{I_{n}^{j}\left(c_{0}\right) \subset \mathcal{V}}\left|S f\left(f^{j}(x)\right)\right|\left(\frac{\left|I_{n}^{j}\left(c_{0}\right)\right|}{\left|I_{n}\left(c_{0}\right)\right|}\right)^{2} \\
& \leq \frac{K_{2} M}{\left|I_{n}\left(c_{0}\right)\right|^{2}} \sum_{I_{n}^{j}\left(c_{0}\right) \subset \mathcal{V}}\left|I_{n}^{j}\left(c_{0}\right)\right|^{2}
\end{aligned}
$$

onde $M=\sup _{y \in \mathcal{V}}|S f(y)|<\infty$ é uma constante que depende só de $f$ (e das vizinhanças $\mathcal{U}, \mathcal{V}$ ). Mas

$$
\sum_{I_{n}^{j}\left(c_{0}\right) \subset \mathcal{V}}\left|I_{n}^{j}\left(c_{0}\right)\right|^{2} \leq\left(\max _{I_{n}^{j}\left(c_{0}\right) \subset \mathcal{V}}\left|I_{n}^{j}\left(c_{0}\right)\right|\right) \sum_{I_{n}^{j}\left(c_{0}\right) \subset \mathcal{V}}\left|I_{n}^{j}\left(c_{0}\right)\right|<\delta_{n} .
$$

Portanto

$$
\left|\Sigma_{2}^{(n)}(x)\right| \leq \frac{K_{2} M \delta_{n}}{\left|I_{n}\left(c_{0}\right)\right|^{2}}
$$

\footnotetext{
${ }^{1}$ Denotamos por int $(X)$ o interior do conjunto $X \subset S^{1}$.
} 
Escolhendo $n_{0}=n_{0}(f)>n_{1}$ suficientemente grande tal que $K_{2} M \delta_{n}<K_{1}$ para todo $n \geq n_{0}$, obtemos de (3.1.18) e (3.1.20) que $S f^{q_{n+1}}(x)<0$ para todo $x \in G_{i}^{*}$, para todo $n \geq n_{0}$.

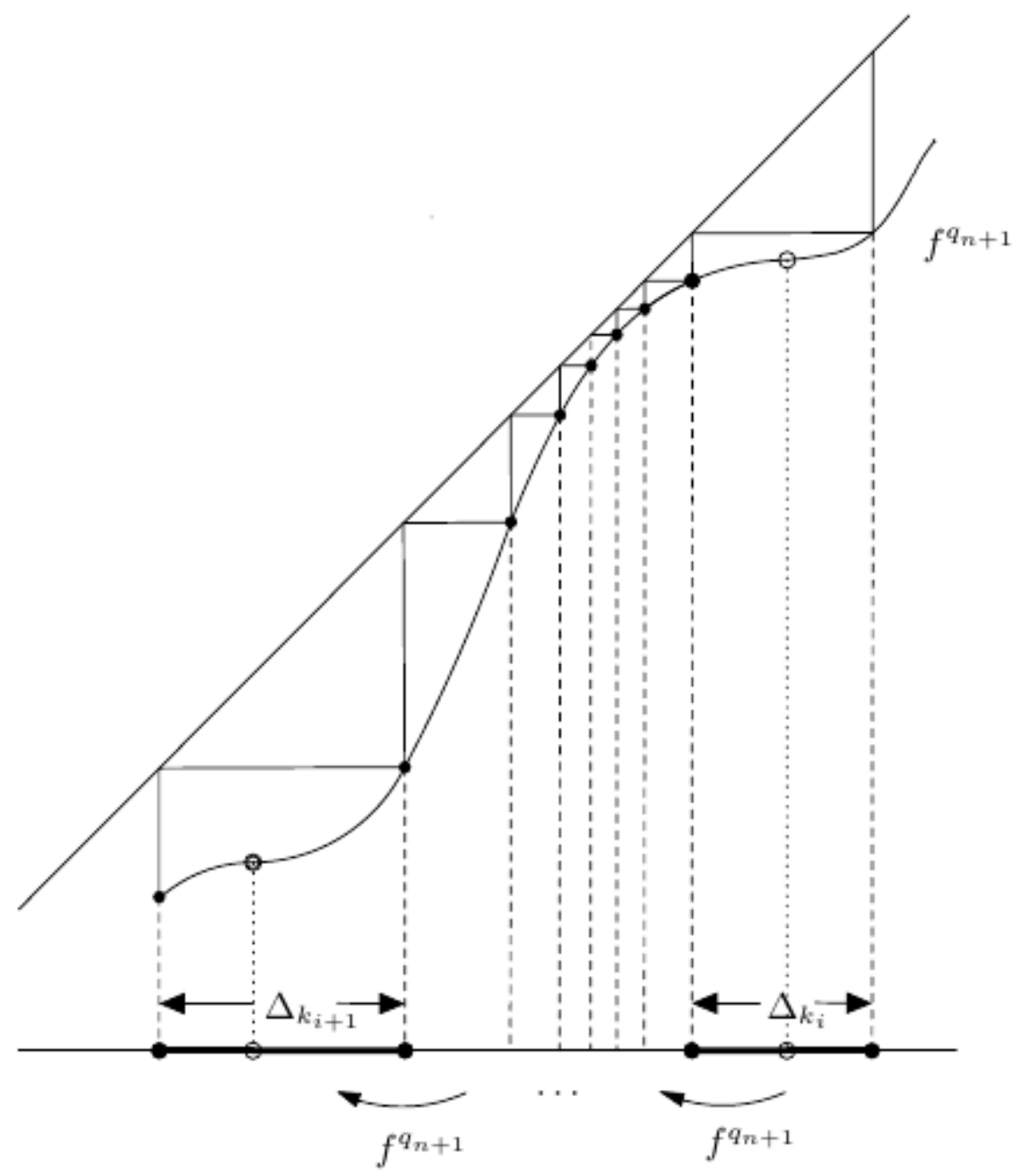

Figura 3.4: Dois sítios críticos consecutivos e o ponte entre eles: imagem dinâmica.

Do lema prévio, podemos deduzir o seguinte resultado correspondente com os pontes $G_{i}, 0 \leq i \leq r$, contidos no intervalo do primeiro retorno $I_{n}\left(c_{0}\right)$ (veja Figura 3.4).

Proposição 3.1.3. Para todo $n \geq n_{0}$, onde $n_{0}$ esta dado pelo Lema 3.1.6, e para todo $i=0,1,2, \ldots, r$ para os quais o ponte reduzido $G_{i}^{*} \subset I_{n}\left(c_{0}\right)$ é não vazio, a restrição

$$
\left.f^{q_{n+1}}\right|_{G_{i}^{*}}: G_{i}^{*} \rightarrow f^{q_{n+1}}\left(G_{i}^{*}\right)
$$

é um mapa quase-parabólico com comprimento $\ell_{i}=k_{i+1}-k_{i}-3$ e largura $\sigma_{i} \geq \sigma$, onde $\sigma=\sigma(C)>0$ depende só da constante $C$ das real bounds.

Demonstração. Por construção, o mapa $\phi=\left.f^{q_{n+1}}\right|_{G_{i}^{*}}$ não tem pontos criticos, assim é um difeomorfismo sob sua imagem. Como $G_{i}^{*}=\bigcup_{k=k_{i}+2}^{k_{i+1}-2} \Delta_{k}$ e $\phi\left(\Delta_{k}\right)=f^{q_{n+1}}\left(\Delta_{k}\right)=\Delta_{k+1}$ para todo $k$, segue-se que o comprimento de $\phi$ é $\ell_{i}=k_{i+1}-k_{i}-3$. Aliás, pelo Lema 3.1.6, também temos que $S \phi=S f^{q_{n+1}}<0$. 
Finalmente, como os intervalos $\Delta_{k_{i}+2}$ e $\Delta_{k_{i+1}-2}$ são comparáveis com $G_{i}^{*}$ (por real bounds e Lema 3.1.3), então a largura de $\phi$ satisfaz a afirmação.

O seguinte corolário é o resultado principal da presente subseção.

Corolário 3.1.1. Para todo $n \in \mathbb{N}$, cada ponte não vazio $G_{i, j}=f^{j}\left(G_{i}\right) \in \mathcal{P}_{n}^{*}\left(c_{0}\right)$ admite uma decomposição balanceada com constantes de comparabilidade uniformes dependendo unicamente das real bounds de $f$.

Demonstração. Aqui assumimos que $n \geq n_{0}$, onde $n_{0}$ é o número natural do Lema 3.1.6. Para pontes primários, ou seja, para os pontes $G_{i, 0}=G_{i} \subset I_{n}\left(c_{0}\right)$ (i.e., aqueles com $j=0$ ), a afirmação segue-se da Proposição 3.1.3, Lema 3.1.5 e Observação 3.1.3. Para pontes segundarias, a saber $G_{i, j}=f^{j}\left(G_{i}\right)$, $1 \leq j \leq q_{n+1}-1$, observamos que o mapa $f^{j}: \operatorname{int}\left(G_{i}\right) \rightarrow \operatorname{int}\left(G_{i, j}\right)$ é um difeomorfismo logo aplicamos o principio de distorção de Koebe (a imagem da decomposição balanceada de $G_{i}$ sob $f^{j}$ gera uma decomposição balanceada de $\left.G_{i, j}\right)$.

\subsection{Prova do Teorema da conjugação quase-simétrica}

Nesta seção, vamos provar finalmente o Teorema 3.0.1. A estratégia que seguimos é a mesma usada em [dFdM99, seção 4] na prova deste mesmo resultado no caso mapas uni-crítico do círculo.

\subsubsection{Uma fine grid adequada}

Vamos definir uma partição auxiliar $\widetilde{\mathcal{P}}_{n}^{*}\left(c_{0}\right)$, para $n \geq 1$. Os intervalos de esta nova partição são os intervalos que não são sela-node de $\mathcal{P}_{n}^{*}\left(c_{0}\right)$, junto com os intervalos das partições balanceadas dos intervalos sela-node de $\mathcal{P}_{n}^{*}\left(c_{0}\right)$. Como mostramos a continuação, a partição $\mathcal{Q}_{n}$ é construída de $\widetilde{\mathcal{P}}_{m}^{*}\left(c_{0}\right)$ e de $\mathcal{P}_{m}^{*}\left(c_{0}\right)$ para vários valores de $m \leq n$.

Proposição 3.2.1. Existe uma fine grid $\left\{\mathcal{Q}_{n}\right\}$ em $S^{1}$ com as seguintes propriedades.

(a) Todo intervalo de $\mathcal{Q}_{n}$ é a união de no máximo $a=4 N+3$ intervalos $^{2}$ de $\mathcal{Q}_{n+1}$.

(b) Todo intervalo $\Delta \in \mathcal{Q}_{n}$ é união de intervalos de $\mathcal{P}_{m}^{*}\left(c_{0}\right)$ para algum $m \leq n$, e existem três possibilidades:

$\left(b_{1}\right) \Delta$ é um único intervalo de $\mathcal{P}_{m}^{*}\left(c_{0}\right)$;

$\left(b_{2}\right) \Delta$ é um intervalo central de $\widetilde{\mathcal{P}}_{m}^{*}\left(c_{0}\right)$;

$\left(b_{3}\right) \Delta$ é união de pelo menos dois intervalos de $\mathcal{P}_{m+1}^{*}\left(c_{0}\right)$ contidos num único intervalo de $\widetilde{\mathcal{P}}_{m}^{*}\left(c_{0}\right)$.

Demonstração. A prova é por indução em $n$. A partição $\mathcal{Q}_{1}$ esta formada por todos os intervalos de $\mathcal{P}_{1}^{*}\left(c_{0}\right)$ que não são sela-node junto com os intervalos $L_{0}, M_{1}$ e $R_{0}$ de cada intervalo sela-node de $I \in \mathcal{P}_{1}^{*}\left(c_{0}\right)\left(I=L_{0} \cup M_{1} \cup R_{0}\right)$. Podemos ver que cada intervalo de $\mathcal{Q}_{1}$ cai dentro de uma das categorias $\left(b_{1}\right)-\left(b_{3}\right)$ no enunciado do Lema.

Agora, assumindo que a partição $\mathcal{Q}_{n}$ esta definida, vamos formar a partição $\mathcal{Q}_{n+1}$ como segue. Tomemos um intervalo $I \in \mathcal{Q}_{n}$ e considere os seguintes quatro casos.

\footnotetext{
${ }^{2}$ Como já vimos em $§ 3.1 .3$, cada intervalo longo $I_{n}^{i}\left(c_{0}\right) \in \mathcal{P}_{n}\left(c_{0}\right)$ é decomposto como a união de $2 r+3 \leq 4 N+3$ intervalos de $\mathcal{P}_{n}^{*}\left(c_{0}\right)$.
} 
(1) Se $I$ é um único intervalo de $\mathcal{P}_{m}^{*}\left(c_{0}\right)$ então uma das seguintes duas opções podem ocorrer:

(i) $I$ é um intervalo sela-node: Neste caso escrevemos $I=L_{0} \cup M_{1} \cup R_{0}$ como acima e tomamos $L_{0}, R_{0}$ e $M_{1}$ como intervalos de $\mathcal{Q}_{n+1}$. Notemos que os intervalos laterais $L_{0}$ e $R_{0}$ são intervalos do tipo $\left(b_{1}\right)$, enquanto o intervalo central $M_{1}$ é de tipo $\left(b_{2}\right)$.

(ii) I não é um intervalo sela-node: Aqui, temos dois sub-casos a considerar. A primeira opção é que $I$ seja um único intervalos de $\mathcal{P}_{m}\left(c_{0}\right)$, neste caso quebramos ele na união de máximo $a$ intervalos de $\mathcal{P}_{m+1}^{*}\left(c_{0}\right)$ e tomamos eles como intervalos de $\mathcal{Q}_{n+1}$, todos eles são do tipo $\left(b_{1}\right)$. A segunda opção é que $I$ seja um ponte, e neste caso quebramos ele em seus $\leq 1,000$ intervalos de $\mathcal{P}_{m+1}\left(c_{0}\right)$ contidos nele. Tomamos estos subintervalos como intervalos de $\mathcal{Q}_{n+1}$, novamente eles são do tipo $\left(b_{1}\right)$.

(2) Se $I$ é um intervalo central de $\widetilde{\mathcal{P}}_{m}^{*}\left(c_{0}\right)$ que não é um intervalo final, consideramos o próximo intervalo central de $\widetilde{\mathcal{P}}_{m}^{*}\left(c_{0}\right)$ no interior de $I$, digamos $M$, e os correspondentes intervalos laterais $L$ e $R$ tal que $I=L \cup M \cup R$, e tomamos $L, R$ e $M$ membros de $\mathcal{Q}_{n+1}$. Notemos que $L$ e $R$ são de tipo $\left(b_{3}\right)$, enquanto $M$ é de tipo $\left(b_{2}\right)$.

(3) Se $I$ é união de $p \geq 2$ intervalos consecutivos $J_{1}, \ldots, J_{p}$ de $\mathcal{P}_{m+1}\left(c_{0}\right)$ dentro de um único intervalo de $\mathcal{P}_{m}^{*}\left(c_{0}\right)$ (isto acontece quando $I$ esta contido num intervalo lateral da decomposição balanceada de um ponte longo), dividimos ele em aproximadamente três partes iguais, i.e. escrevemos $p=$ $2 q+r$, onde $r=0$ ou 1 , e consideremos $I=L \cup R$ onde

$$
L=\bigcup_{j=1}^{q} J_{j}, R=\bigcup_{j=q+1}^{p} J_{j} .
$$

Assim, obtemos de esta forma dois intervalos novos de $\mathcal{Q}_{n+1}(L$ e $R)$ os quais são ou intervalos $\mathcal{P}_{m+1}\left(c_{0}\right)$, e portanto do tipo $\left(b_{1}\right)$, ou uma vez mais intervalos do tipo $\left(b_{3}\right)$.

O anterior completa a indução. O fato que $\left\{\mathcal{Q}_{n}\right\}_{n \geq 1}$ forma uma fine grid segue-se das real bounds, Lema 3.1.5, Proposição 3.1.2 e Corolário 3.1.1. Logo, só temos que verificar que a condição (c) na definição 3.0.3 é satisfeita por alguma constante $\rho>1$ que só depende das real bounds). Dados dois intervalos adjacentes $\Delta, \Delta^{\prime} \in \mathcal{Q}_{n}$, temos dois casos a considerar.

(a) Existe $m, m^{\prime} \leq n$ tal que $\Delta$ é um único intervalo de $\mathcal{P}_{m}\left(c_{0}\right)$ e $\Delta^{\prime}$ é um único intervalo de $\mathcal{P}_{m^{\prime}}\left(c_{0}\right)$. Neste caso, $m=m^{\prime}$, ou $m$ e $m^{\prime}$ difere de 1 (isto pode ser provado por indução em $n$ da construção de $\mathcal{Q}_{n}$ dada acima). Mas então temos $|\Delta| \asymp\left|\Delta^{\prime}\right|$ pelas real bounds (Teorema 2.2.2).

(b) Para algum $m \leq n$, pelo menos um dos dois intervalos, digamos $\Delta$, é a união de $p \geq 2$ intervalos de $\mathcal{P}_{m+1}\left(c_{0}\right)$ dentro de um único intervalo de $\mathcal{P}_{m}^{*}\left(c_{0}\right)$, o qual é necessariamente um ponte. Isto implica que tanto $\Delta$ como $\Delta^{\prime}$ estão contidas no mesmo ponte $G \in \mathcal{P}_{m}^{*}\left(c_{0}\right)$. Olhando para a decomposição balanceada de $G$ (dada pelo Corolário 3.1.1), observamos que se apresentam duas possibilidades. A primeira é que tanto $\Delta$ como $\Delta^{\prime}$ estão contidos no mesmo intervalo lateral $\left(L_{i}, R_{i}\right)$ ou o mesmo intervalo central $\left(M_{i}\right)$ da dita decomposição balanceada. Neste caso, $\Delta$ e $\Delta^{\prime}$ são uniões do mesmo numero de domínios fundamentais de $G$, e portanto $|\Delta| \asymp\left|\Delta^{\prime}\right|$ pelo Lema 3.1.5 e pela Proposição 3.1.2. A segunda possibilidade é que $\Delta$ e $\Delta^{\prime}$ estejam contidos em intervalos adjacentes da decomposição balanceada de $G$. Neste casso, um dos dois intervalos, $\Delta$ ou $\Delta^{\prime}$, é união de no máximo o dobro dos domínios fundamentais de $G$ como o outro, e assim, $|\Delta| \asymp\left|\Delta^{\prime}\right|$, novamente pelo Lema 3.1.5 e a Proposição 3.1.2.

Isto estabelece a comparabilidade de todos os átomos adjacentes de $\mathcal{Q}_{n}$, com constantes dependendo só das cotas reais, e portanto a prova esta completa. 


\subsubsection{Prova do Teorema 3.0.1}

Prova do Teorema 3.0.1. Sejam $\left\{\mathcal{Q}_{n}(f)\right\}$ e $\left\{\mathcal{Q}_{n}(g)\right\}$ as fine grids dadas pela Proposição 3.2 .1 para os mapas $f$ e $g$, respectivamente. Por hipóteses a conjugação $h$ leva cada ponto crítico de $f$ no correspondente ponto crítico de $g$, então $h$ leva cada sítio crítico de $\mathcal{P}_{m}^{*}\left(c_{0}(f)\right)$ no correspondente sítio crítico de $\mathcal{P}_{m}^{*}\left(c_{0}(g)\right)$, e o mesmo para pontes, para todo $m \geq 1$. Logo, $h$ é um homeomorfismo entre as duas fine grids e portanto $h$ é quase-simétrica, pelo critério 3.0.1.

\subsubsection{Comentários sob a quase-simetria para mapas com quantidade diferente de pontos críticos}

Se $f$ e $g$ são dois mapas multicríticos do círculo com mesmo número de rotação de tipo limitado, então a conjugação entre eles que leva ponto crítico em ponto crítico é quase-simétrica inclusive se a quantidade de pontos críticos dos mapas é diferente. Isto é verdade pois as partições dinâmicas formam a fine grid procurada e pelo Teorema das Real Bounds a desigualdade 3.0.2 é válida. Consequentemente a Proposição 3.0.1 implica que a conjugação que leva pelo menos um ponto crítico em outro é quasesimétrica.

Da prova dada neste capítulo, i.é., da construção da fine grid para número de rotação irracional arbitrário, é indispensável que a quantidade de pontos críticos dos dois mapas seja a mesma. Lembremos que a conjugação constitui um homeomorfismo entre fine grides. De fato, existem exemplos de mapas multicríticos do círculo com número de rotação irracional arbitrário que não podem ser conjugados por um mapa quase-simétrico, veja [dFG]. 


\section{Capítulo 4}

\section{Cotas beau para mapas multicríticos do círculo}

No contexto de renormalização, a suavidade da conjugação entre dois mapas uni-críticos do círculo segue-se da convergência exponencial de suas respectivas renormalizações. Para isto é necessário que a constante do Teorema 2.2.2 seja assintoticamente a mesma para os dois mapas. Este tipo de constantes são chamadas pelo Sullivan [Sul88] de constantes beau, i.e. as cotas nos scalling ratios das orbitas críticas tornar-se assintoticamente universais e independente do mapa. Neste capitulo vamos mostrar que mapas multicríticos do círculo na mesma classe topológica, satisfazem o teorema 2.2.2 para a mesma constante, em partiçôes dinâmicas profundas.

Cabe ressaltar, que uma prova de este resultado no caso uni-crítico se encontra no artigo [dFdM99]. De fato, nossa prova para o caso multicrítico é uma adaptação do caso uni-crítico. Este capítulo esta baseado no artigo [EdFG16].

Neste capítulo $f$ denotará um mapa multicrítico do círculo e classe $C^{3}$ com número de rotação $\rho(f) \in \mathbb{R} \backslash \mathbb{Q}$. Denotaremos por $c_{0}, c_{1}, \cdots, c_{N-1}$ e por $d_{0}, d_{1}, \cdots, d_{N-1}$ seus pontos críticos e suas criticalidades, respectivamente. A continuação, vamos fixar um ponto crítico, digamos $c_{0}$ e em sua partição dinâmica associada, i.e. $\mathcal{P}_{n}\left(c_{0}\right)$ para $n \geq 0$. Para simplificar a notação, vamos escrever $\mathcal{P}_{n}$ ao invés de $\mathcal{P}_{n}\left(c_{0}\right)$. Consequentemente, os intervalos $I_{n}^{i}\left(c_{0}\right)$ e $I_{n+1}^{j}\left(c_{0}\right)$ serão denotados por $I_{n}^{i}$ e $I_{n+1}^{j}$, respectivamente. Além disso, para o intervalo $J \in \mathcal{P}_{n}$ vamos denotar por $J^{*}$ a união de $J$ com os intervalos adjacentes de $\mathcal{P}_{n}$ à direita e à esquerda de J. Vamos assumir, para n suficientemente grande, que todo intervalo de $\mathcal{P}_{n}$ contém no máximo um ponto crítico de $f$.

\subsection{As $\operatorname{cotas} C^{1}$}

Como o mapa $f$ possui mais de um ponto crítico, entâo a derivada de algum iterado não esta sempre longe de zero em alguns pontos do círculo. Não obstante, é possível limitar por cima a derivada de qualquer iterado em todos os pontos no domínio. O objetivo de esta seção é provar a seguinte afirmação.

Lema 4.1.1. Dado $f$ mapa multicrítico do círculo. Existem duas constantes $K=K(f)>1$ e $n_{0}=$ $n_{0}(f) \in \mathbb{N}$ tal que para todo $n>n_{0}, x \in I_{n}$ e $j \in\left\{0,1, \cdots, q_{n+1}\right\}$, temos que

$$
D f^{j}(x) \leq K \cdot \frac{\left|f^{j}\left(I_{n}\right)\right|}{\left|I_{n}\right|}
$$


Como consequência, obtemos o seguinte resultado,

Corolário 4.1.1. A sequência $\left\{f^{q_{n+1}} \mid I_{n}\right\}_{n \geq 1}$ é limitada na métrica $C^{1}$.

Demonstração. Pela combinatória, temos que $I_{n+1} \subset f^{q_{n+1}}\left(I_{n}\right) \subset I_{n} \cup I_{n+1}$. Consequentemente:

$$
\frac{\left|I_{n+1}\right|}{\left|I_{n}\right|} \leq \frac{\left|f^{q_{n+1}}\left(I_{n}\right)\right|}{\left|I_{n}\right|} \leq 1+\frac{\left|I_{n+1}\right|}{\left|I_{n}\right|}
$$

Pelo Teorema das real bounds 2.2.2, $\left|I_{n+1}\right| \asymp\left|I_{n}\right|$ com constante de comparabilidade igual a $C$. Portanto, $\left|f^{q_{n+1}}\left(I_{n}\right)\right| \asymp\left|I_{n}\right|$ com constante de comparabilidade igual a $1+C$. Agora, pelo Lema 4.1.1 e usando o Teorema do valor meio, obtemos, $\sup _{x \in I_{n}} D f^{q_{n+1}}(x) \leq K(1+C)$.

\subsubsection{Prova do Lema 4.1.1}

Para cada $n \in \mathbb{N}$ consideremos os intervalos $L_{n}=I_{n+1}, R_{n}=f^{q_{n}}\left(I_{n}\right)$ e $T_{n}=L_{n} \cup I_{n} \cup R_{n}$. Temos as seguintes observações preliminares:

Fato 4.1.1. A família $\left\{T_{n}, f\left(T_{n}\right), \cdots, f^{q_{n+1}-1}\left(T_{n}\right)\right\}$ tem multiplicidade de interseção limitada por 3.

O fato 4.1.1 segue-se da seguinte observação: dados $z \in S^{1}$ e $n \in \mathbb{N}$ let $I=\left[z, R_{\rho}^{3 q_{n}}(z)\right]$, onde $R_{\rho}$ é a rotação rígida de ângulo $2 \pi \rho$ no círculo unitário. Então a multiplicidade de interseção da familia $\left\{I, R_{\rho}(I), \ldots, R_{\rho}^{q_{n+1}-1}(I)\right\}$ é 3 para todo $n \in \mathbb{N}$.

Fato 4.1.2. Existe $\tau>0$ (constante que depende só das real bounds de f) tal que

$$
\left|L_{n}^{j}\right|>\tau\left|I_{n}^{j}\right| \quad \text { and } \quad\left|R_{n}^{j}\right|>\tau\left|I_{n}^{j}\right|
$$

para cada $j \in\left\{0, \cdots, q_{n+1}\right\}$ e para todo $n \in \mathbb{N}$.

Prova do Fato 4.1.2. Se $j=0$, observe que os intervalos $L_{n}, I_{n}$ e $R_{n}$ são adjacentes e pertencem à partição dinâmica $\mathcal{P}_{n}$, então pelo Teorema das real bounds eles são comparáveis por uma constante que só depende de $f$. Agora vamos mostrar que para $j=q_{n+1}$ estes três intervalos também são comparáveis.

Por um lado, os intervalos $I_{n+1}$ e $I_{n+1}^{q_{n+1}}$ pertencem à $\mathcal{P}_{n+1}$ e são adjacentes, assim eles são comparáveis pelas real bounds. Além disso, $I_{n+1} \subset I_{n}^{q_{n+1}} \subset I_{n+1} \cup I_{n}$. Portanto, $\left|I_{n}^{q_{n+1}}\right| \asymp\left|I_{n+1}^{q_{n+1}}\right|$, i.e.

$$
\left|L_{n}^{q_{n+1}}\right| \asymp\left|I_{n}^{q_{n+1}}\right| .
$$

Por outro lado, como os intervalos $I_{n}$ e $I_{n}^{q_{n}}$ são adjacentes e pertencem à $\mathcal{P}_{n}$ então eles são comparáveis. Aliás,

$$
I_{n+1}^{q_{n}} \subset I_{n}^{q_{n}+q_{n+1}} \subset I_{n} \cup I_{n}^{q_{n}} .
$$

Pela prova do Teorema das real bounds 2.2 .2 , i.e. apêndice B, sabemos que $\left|I_{n+1}^{q_{n}}\right| \asymp\left|I_{n}\right|$ e assim $\left|I_{n}^{q_{n}+q_{n+1}}\right| \asymp\left|I_{n}\right|$. Mas $I_{n+1} \subset I_{n}^{q_{n+1}} \subset I_{n} \cup I_{n+1}$, novamente pelas real bounds obtemos

$$
\left|R_{n}^{q_{n+1}}\right|=\left|I_{n}^{q_{n}+q_{n+1}}\right| \asymp\left|I_{n}\right| \asymp\left|I_{n}^{q_{n+1}}\right| .
$$

Seja $1 \leq j \leq q_{n+1}-1$. Consideremos os intervalos $\left|L_{n}^{j}\right|,\left|I_{n}^{j}\right|,\left|R_{n}^{j}\right|$ e suas imagens pelo mapa $f^{q_{n+1}-j}$. Pela desigualdade de Cross-Ratio (veja 2.2.2 e apêndice A) e o Fato 4.1.1 sabemos que existe 
uma constante $K=K(f)>1$ tal que

$$
\frac{\left|L_{n}^{q_{n+1}}\right|\left|R_{n}^{q_{n+1}}\right|\left|L_{n}^{j} \cup I_{n}^{j}\right|\left|I_{n}^{j} \cup R_{n}^{j}\right|}{\left|L_{n}^{j}\right|\left|R_{n}^{j}\right|\left|L_{n}^{q_{n+1}} \cup I_{n}^{q_{n+1}}\right| \mid I_{n}^{q_{n+1}} \cup R_{n}^{q_{n+1} \mid}} \leq K .
$$

Por (4.1.2) e (4.1.3) na última desigualdade, obtemos

$$
\left(1+\frac{\left|I_{n}^{j}\right|}{\left|L_{n}^{j}\right|}\right)\left(1+\frac{\left|I_{n}^{j}\right|}{\left|R_{n}^{j}\right|}\right) \leq K
$$

e assim temos provado o Fato 4.1.2.

Observação 4.1.1. Note que existe $n_{0}=n_{0}(f)$, dado pelo Lema 4.1.1, tal que para todo $n \geq n_{0}$ $\operatorname{Card}\left(f^{j}\left(T_{n}\right) \cap \operatorname{Crit}(f)\right) \leq 1$. Isto é porque, pela minimalidade, $\left|f^{j}\left(T_{n}\right)\right|$ vai para zero quando $n$ vai para infinito.

Definição 4.1.1 (Tempos Críticos). Dizemos que $j \in\left\{1, \cdots, q_{n+1}\right\}$ é um tempo crítico de $f$, se $f^{j}\left(T_{n}\right) \cap \operatorname{Crit}(f) \neq \varnothing$.

Observação 4.1.2. Note que $\operatorname{Card}(\{$ tempos críticos $\}) \leq 3 N$.

Fato 4.1.3. Sejam $1 \leq j_{1}<j_{2} \leq q_{n+1}$ dois tempos críticos consecutivos de $f$. Então para todo $x \in f^{j_{1}+1}\left(I_{n}\right)$ temos,

$$
D f^{j_{2}-j_{1}-1}(x) \asymp \frac{\left|f^{j_{2}}\left(I_{n}\right)\right|}{\left|f^{j_{1}+1}\left(I_{n}\right)\right|},
$$

com as constantes de comparabilidade dependendo só das real bounds.

Prova do Fato 4.1.3. Observemos que $f^{j_{2}-j_{1}-1}: f^{j_{1}+1}\left(T_{n}\right) \rightarrow f^{j_{2}}\left(T_{n}\right)$ é um difeomorfismo. O Fato 4.1.1 implica que $\sum_{i=0}^{j_{2}-j_{1}-1}\left|f^{i}\left(f^{j_{1}+1}\left(T_{n}\right)\right)\right|<3$, e pelo Fato 4.1.2 o intervalo $f^{j_{2}-j_{1}-1}\left(f^{j_{1}+1}\left(T_{n}\right)\right)$ contém uma vizinhança $\tau$-escalada de $f^{j_{2}-j_{1}-1}\left(f^{j_{1}+1}\left(I_{n}\right)\right)$. Pelo principio de distorção de Koebe (veja Lema A.2.1 no apêndice A) existe uma constante $K_{0}=K_{0}(f)>1$ tal que para todo $x, y \in f^{j_{1}+1}\left(I_{n}\right)$ temos que

$$
\frac{1}{K_{0}} \leq \frac{\left|D f^{j_{2}-j_{1}-1}(x)\right|}{\left|D f^{j_{2}-j_{1}-1}(y)\right|} \leq K_{0}
$$

Seja $y \in I_{n}^{j_{1}+1}$ o ponto dado pelo Teorema do Valor Meio tal que $D f^{j_{2}-j_{1}-1}(y)=\frac{\left|f^{j_{2}}\left(I_{n}\right)\right|}{\left|f^{j_{1}+1}\left(I_{n}\right)\right|}$. Então para todo $x \in f^{j_{1}+1}\left(I_{n}\right)$,

$$
\frac{1}{K_{0}} \frac{\left|f^{j_{2}}\left(I_{n}\right)\right|}{\left|f^{j_{1}+1}\left(I_{n}\right)\right|} \leq D f^{j_{2}-j_{1}-1}(x) \leq K_{0} \frac{\left|f^{j_{2}}\left(I_{n}\right)\right|}{\left|f^{j_{1}+1}\left(I_{n}\right)\right|}
$$

A prova do Lema 4.1.1 segue-se da combinação do Fato 4.1.3 junto com o item (3) no Lema 2.2.1 e a regra da cadeia:

$$
D f^{j}(x) \leq(3 d)^{3 N} K_{0}^{3 N} \frac{\left|f^{j}\left(I_{n}\right)\right|}{\left|I_{n}\right|} \quad \text { para todo } x \in I_{n} \text { e } j \in\left\{1, \cdots, q_{n+1}\right\},
$$


onde $N$ é o número de pontos críticos de $f$, d é o máximo das criticalidades destes pontos críticos e $K_{0}=K_{0}(f)$ é dado pelo Fato 4.1.3.

\subsection{Derivada Schwarziana negativa do mapa de primeiro retorno}

Nesta seç̧ão vamos provar que os iterados de uma vizinhança do ponto crítico $c_{0}$, incluindo o iterado do primeiro retorno, tem derivada Schwarziana negativa em seus pontos regulares. O número $n_{0}=n_{0}(f) \in \mathbb{N}$ é o mesmo dado pelo Lema 4.1.1.

Lema 4.2.1. Seja $f$ um mapa multicrítico do círculo. Existe $n_{1}=n_{1}(f) \in \mathbb{N}$ tal que para todo $n \geq n_{1}$ temos que

$$
S f^{j}(x)<0 \quad \text { para todo } j \in\left\{1, \cdots, q_{n+1}\right\} \text { e para todo } x \in I_{n} \text { ponto regular de } f^{j} .
$$

Do mesmo jeito,

$$
S f^{j}(x)<0 \quad \text { para todo } j \in\left\{1, \cdots, q_{n}\right\} \text { e para todo } x \in I_{n+1} \text { ponto regular de } f^{j} .
$$

Prova do Lema 4.2.1. A prova que damos a continuação segue a exposição em [dFdM99, páginas 380381]. Vamos provar o lema somente para $x \in I_{n}$ ponto regular de $f^{j} \operatorname{com} j \in\left\{1, \cdots, q_{n+1}\right\}$ (o outro caso é análogo).

Pelo item (1) do Lema 2.2.1, para cada ponto crítico $c_{i}$ existe uma vizinhança $U_{i} \subseteq S^{1}$ e uma constante positiva $K_{i}$ tal que para cada $x \in U_{i} \backslash\left\{c_{i}\right\}$ temos

$$
S f(x)<-\frac{K_{i}}{\left(x-c_{i}\right)^{2}}<0 .
$$

Seja $\mathcal{U}=\bigcup_{i=0}^{i=N-1} U_{i}$, e $\mathcal{V} \subset S^{1}$ conjunto aberto que não contém nenhum ponto crítico de $f$ e tal que $\mathcal{U} \cup \mathcal{V}=S^{1}$. Notemos que $M=\sup _{y \in \mathcal{V}}|S f(y)|$ é finito. Seja $\delta_{n}=\max _{0 \leq j<q_{n+1}}\left|I_{n}^{j}\right|$. Sabemos que $\delta_{n} \rightarrow 0$ quando $n \rightarrow \infty$, pois $f$ é topologicamente conjugado à rotação. Escolhemos $n_{1}=n_{1}(f)>n_{0}$ suficientemente grande tal que $\delta_{n}$ é mais pequeno que o número de Lebesgue do cobrimento $\{\mathcal{U}, \mathcal{V}\}$ do círculo para todo $n \geq n_{1}$. Usando a regra da cadeia da derivada Schwarziana temos que

$$
S f^{j}(x)=\sum_{k=0}^{j-1} S f\left(f^{k}(x)\right)\left[D f^{k}(x)\right]^{2}
$$

para todo $n \geq n_{1}$ e todo $x \in I_{n}$ ponto regular de $f^{j}$. Agora, vamos decompor esta suma como $\Sigma_{1}^{(n)}(x)+\Sigma_{2}^{(n)}(x)$ onde

$$
\Sigma_{1}^{(n)}(x)=\sum_{k: I_{n}^{k} \subset \mathcal{U}} S f\left(f^{k}(x)\right)\left[D f^{k}(x)\right]^{2}
$$

e $\Sigma_{2}^{(n)}(x)$ é a suma sob os termos restantes.

Agora vamos proceder através das seguintes etapas:

(i) Como $I_{n} \subset \mathcal{U}$, a suma no lado direito de (4.2.3) inclui o termo para $k=0$, a saber $S f(x)$. Como todos os outros termos em (4.2.3) também são negativos, e dado que $\left|x-c_{0}\right| \leq\left|I_{n}\right|$, deduzimos 
de (4.2.1) que

$$
\Sigma_{1}^{(n)}(x)<-\frac{K_{1}}{\left|I_{n}\right|^{2}}
$$

(ii) Observemos que

$$
\left|\Sigma_{2}^{(n)}(x)\right| \leq \sum_{I_{n}^{k} \subset \mathcal{V}}\left|S f\left(f^{k}(x)\right)\right|\left[D f^{k}(x)\right]^{2} .
$$

Pelo Lema 4.1.1 existe $K=K(f)>1$ tal que

$$
\begin{aligned}
\left|\Sigma_{2}^{(n)}(x)\right| & \leq \sum_{I_{n}^{k} \subset \mathcal{V}}\left|S f\left(f^{k}(x)\right)\right| K^{2} \frac{\left|I_{n}^{k}\right|^{2}}{\left|I_{n}\right|^{2}} \\
& \leq M \frac{K^{2}}{\left|I_{n}\right|^{2}} \sum_{I_{n}^{k} \subset \mathcal{V}}\left|I_{n}^{k}\right|^{2} \\
& \leq M \frac{K^{2}}{\left|I_{n}\right|^{2}} \max _{0 \leq k \leq j-1}\left|I_{n}^{k}\right| \sum_{I_{n}^{k} \subset \mathcal{V}}\left|I_{n}^{k}\right| \\
& \leq M \frac{K^{2}}{\left|I_{n}\right|^{2}} \delta_{n} .
\end{aligned}
$$

Escolhendo $n_{1}$ suficientemente grande tal que $K^{2} M \delta_{n}<K_{1}$ para todo $n \geq n_{1}$, deduzimos das desigualdades (4.2.4) e (4.2.6) que $S f^{j}(x)<0$ para todo $j \in\left\{1, \cdots, q_{n+1}\right\}$ e para $n \geq n_{1}$.

\subsection{Beau bounds}

O termo beau bounds foi introduzido pelo Sullivan em [Sul88] referia a constantes que assimptoticamente são universais. O objetivo nesta secção é provar que as constantes dadas no teorema 2.2.2 são beau, mais precisamente vamos provar o seguinte resultado:

Teorema 4.3.1 (Beau bounds). Dado $N \geq 1$ em $\mathbb{N}$ e $d>1$ existe uma constante $C=C(N, d)>1$ com a seguinte propriedade: dado um mapa multicrítico do círculo $f$, com máximo $N$ pontos críticos cujas criticalidades são limitadas por d, existe $n_{0}=n_{0}(f) \in \mathbb{N}$ tal que para todo $n \geq n_{0}$ e todo par de intervalos adjacentes $I, J$ em $\mathcal{P}_{n}$ temos

$$
\frac{|J|}{C} \leq|I| \leq C|J|
$$

Uma consequência imediato do Teorema 4.3.1 é a seguinte afirmação.

Corolário 4.3.1 (Corolário do Teorema 4.3.1). Existe $\mu_{1} \in(0,1)$ tal que para todo $x \in S^{1}$ e para todo $n \geq 0$, se $\mathcal{P}_{n}[x]$ representa o intervalo em $\mathcal{P}_{n}$ que contém o ponto $x$ então

$$
\left|\mathcal{P}_{n+1}[x]\right|<\mu_{1}\left|\mathcal{P}_{n-1}[x]\right|
$$

A prova do Teorema 4.3.1 segue a estrategia usada em [dFdM99], mas adaptada no caso de vários pontos críticos. Nas seguintes subsecçôes vamos decompor os iterados de $f$ como mapas com distorção de cross-ratio limitado por cima, com isto vamos reprovar a Desigualdade de Cross-ratio, ou Lema 2.2.2, o que implica o Teorema 4.3.1. 


\subsubsection{Decomposição dos iterados de $f$}

Nesta subsecção $c_{i}$ denota algum ponto crítico do mapa multicrítico do círculo $f$, e $U_{i}$ a vizinhança de $c_{i}$ dada pelo Lema 2.2.1. Aliás, $n_{1} \in \mathbb{N}$ é o número natural dado pelo Lema 4.2.1.

Lema 4.3.1. Dado $\varepsilon>0$ existe $n_{2} \in \mathbb{N}, n_{2}=n_{2}(\varepsilon, f)>n_{1}$, que satisfaz a seguinte propriedade: dado $n \geq n_{2}, \Delta \in \mathcal{P}_{n}$ e $k \in \mathbb{N}$ tal que $f^{j}(\Delta)$ esta contido num elemento de $\mathcal{P}_{n}$ para todo $1 \leq j \leq k$, podemos escrever

$$
f^{k} \mid \Delta^{*}=\phi_{k} \circ \phi_{k-1} \circ \ldots \circ \phi_{1}
$$

onde:

1. Para máximo $3 N+1$ valores de $i \in\{1, \ldots, k\}$, o mapa $\phi_{i}$ é um difeomorfismo com distorção limitada por $1+\varepsilon$.

2. Para máximo $3 N$ valores de $i \in\{1, \ldots, k\}$, o mapa $\phi_{i}$ é a restrição de $f$ a algum intervalo contido em $U_{i}$.

3. Para os restastes valores de $i$, o mapa $\phi_{i}$ é ou a identidade ou um difeomorfismo com derivada Schwarziana negativa.

Na prova seguimos a exposição dada em [dFdM99, paginas 352-353].

Prova do Lema 4.3.1. Seja $C_{0}=C_{0}(f) \geq 1$ a constante dada pelo Principio de distorção de Koebe (Lema A.2.1). Seja $C>1$ e $\mu \in(0,1)$ dados pelo Teorema 2.2.2. Seja $\delta \in(0,1)$ constante tal que $(1+\delta)^{2} \exp \left(C_{0} \delta\right)<1+\varepsilon$, e seja $n_{2} \in \mathbb{N}$ tal que

$$
n_{2} \gg n_{1}+\frac{4 \log \left(\delta \mu^{3 / 2} / C\right)}{\log \mu} .
$$

Notemos que $0<\left(\mu^{1 / 4}\right)^{n_{2}-n_{1}}<\delta \mu^{3 / 2} / C$. Dado $n \geq n_{2}$ definimos $m=m(n)=\left\lfloor\frac{n+n_{1}}{2}\right\rfloor$. Seja $\Delta$ e $k$ como no enunciado, consideremos $J_{m} \in \mathcal{P}_{m}$ tal que $\Delta \subseteq J_{m}$ e além disso, $J_{n_{1}} \in \mathcal{P}_{n_{1}}$ com $J_{m} \subseteq J_{n_{1}}$. Tomando $n$ suficientemente grande, podemos assumir que $\Delta^{*} \subset J_{m}$.

Seja $s \geq 0$ o menor número natural tal que $f^{s}\left(J_{n_{1}}\right)$ contém um ponto crítico de $f$.

Afirmação 4.3.1. A distorção de $f^{s}$, no intervalo $\Delta^{*}$, esta limitada por cima por $1+\varepsilon$.

Prova da Afirmação 4.3.1. Substituindo $n_{1}$ por $n_{1}+1$ se for necessário, podemos assumir que $f^{j}\left(J_{n_{1}}\right) \in$ $\mathcal{P}_{n_{1}}$ para todo $j \in\{0, \ldots, s-1\}$. Pelo Teorema da real bounds, o espaço $\tau$ do intervalo $\Delta^{*}$ no interior de $J_{m}^{*}$ esta limitado por cima por:

$$
\tau \geq \frac{1}{C} \frac{\left|J_{m}^{*}\right|}{\left|\Delta^{*}\right|} \geq \frac{1}{C}\left(\frac{1}{\mu}\right)^{\lfloor(n-m) / 2\rfloor}>\frac{\mu}{C}\left(\frac{1}{\mu}\right)^{(n-m) / 2} .
$$

Como $m \leq \frac{n+n_{1}}{2}$, temos que $n-m \geq n-\frac{n+n_{1}}{2}=\frac{n-n_{1}}{2}$ e então

$$
\frac{1}{\tau} \leq \frac{C}{\mu} \mu^{(n-m) / 2} \leq \frac{C}{\mu}\left(\mu^{1 / 4}\right)^{n-n_{1}}<\sqrt{\mu} \delta<\delta .
$$


Agora, vamos estimar a suma $\ell$ dos comprimentos dos iterados de $J_{m}^{*}$ entre 1 e $s$. Dado que $\frac{n+n_{1}}{2}<m+1$ temos que $m-n_{1}>\frac{n-n_{1}}{2}-1$, e assim para todo $j \in\{0, \cdots, s\}$ obtemos

$$
\begin{aligned}
\left|f^{j}\left(J_{m}^{*}\right)\right| & \leq \mu^{\left\lfloor\left(m-n_{1}\right) / 2\right\rfloor}\left|f^{j}\left(J_{n_{1}}^{*}\right)\right| \leq\left(\mu^{1 / 4}\right)^{n-n_{1}}\left(\frac{1}{\mu}\right)^{3 / 2}\left|f^{j}\left(J_{n_{1}}^{*}\right)\right| \\
& \leq \frac{\delta}{C}\left|f^{j}\left(J_{n_{1}}^{*}\right)\right| .
\end{aligned}
$$

Portanto

$$
\ell=\sum_{j=0}^{j=s}\left|f^{j}\left(J_{m}^{*}\right)\right|<\frac{3 \delta}{C}<\delta,
$$

pois pela combinatória $\sum_{j=0}^{j=s}\left|f^{j}\left(J_{n_{1}}^{*}\right)\right|<3$.

Das desigualdades (4.3.1), (4.3.2) e pelo principio de distorção de Koebe (A.2.1) obtemos que a distorção no intervalo $\Delta^{*}$ esta limitada por cima por

$$
(1+\delta)^{2} \exp \left(C_{0} \delta\right)<1+\varepsilon
$$

Para provar o Lema 4.3.1 vamos decompor a orbita de $\Delta^{*}$ sob $f$ de acordo com o seguinte algoritmo. Para todo $i \in\{0,1, \ldots, k-1\}$ temos duas opções:

1. Se $f^{i}\left(J_{n_{1}}\right)$ não contém ponto crítico de $f$, definimos o mapa $\phi$ como $f^{s}$, onde $s \geq 1$ é o menor número natural tal que $f^{i+s}\left(J_{n_{1}}\right)$ contém um ponto crítico de $f$. Argumentando como no Fato 4.3.1 acima, observamos que este casso satisfaz o item 1) no enunciado.

2. Se $f^{i}\left(J_{n_{1}}\right)$ contém um ponto crítico $c$ de $f$ vamos assumir, tomando $n_{2}$ suficientemente grande, que $f^{i}\left(\Delta^{*}\right) \subset I_{n_{1}}(c) \cup I_{n_{1}+1}(c)$. Então temos duas opções:

(2.1) Se $f^{i}\left(\Delta^{*}\right)$ não contém este ponto crítico (e portanto nenhum outro) seja $s \geq 1$ o menor natural tal que $f^{i+s}\left(\Delta^{*}\right)$ contém um ponto crítico de $f$, definimos o mapa $\phi$ como $f^{s}$. Pelo Lema 4.2.1 (e como a composição de difeomorfismos com derivada Schwarziana negativa é um difeomorfismo com derivada Schwarziana negativa) este casso satisfaz o item 3) no enunciado.

(2.2) Se o ponto crítico pertence a $f^{i}\left(\Delta^{*}\right)$ definimos o mapa $\phi$ como um iterado de $f$ (e assim, este mapa satisfaz o item 2) no enunciado).

Note que, pela combinatória, a primeira opção acontece máximo $3 N+1$ vezes, enquanto que a segunda acontece máximo $3 N$ vezes.

Corolário 4.3.2. Dado $N \geq 1$ natural e $d>1$, existe uma constante $C=C(N, d)>1$ com a seguinte propriedade: dado $f$ mapa multicrítico do círculo, com $N$ pontos críticos cujas criticalidades estão limitadas por $d$, existe $n_{3}=n_{3}(f)$ tal que para $n \geq n_{3}, \Delta \in \mathcal{P}_{n}$ e $k \in \mathbb{N}$ tal que $f^{j}(\Delta)$ esta contido num elemento de $\mathcal{P}_{n}$ para todo $1 \leq j \leq k$, temos:

$$
\operatorname{CrD}\left(f^{k} ; \Delta, \Delta^{*}\right) \leq C .
$$


A prova do Corolário 4.3.2 segue-se da decomposição obtida no Lema 4.3.1, combinando a Observação A.2.1 no apêndice A, Lema A.2.2 e Lema 2.2.1 item (4). Observemos que a constante $C$ depende só $d a$ quantidade de pontos críticos e $f$ e de seus ordens, mas não de $f$ mesmo. De fato, basta considerar $C=(1+1 / 2)^{2(3 N+1)}\left(9 d^{2}\right)^{3 N}$.

\subsection{Prova do Teorema 4.3.1}

A prova das real bounds, Teorema 4.3.1, é a mesma prova do Teorema das Real Bounds 2.2.2 dada em [EdF15, Seção 3, p. 8-16] ou no apêndice B de esta tese, só que nesta vez substituímos a desigualdade de Cross-Ratio 2.2.2 pelo Corolário 4.3.2. 


\section{Capítulo 5}

\section{Considerações finais}

Como mencionamos no final do capítulo 3, para que a conjugação entre dois mapas multicríticos seja pelo menos quase-simétrica, precisamos que a quantidade de pontos críticos seja a mesma para os dois mapas. De fato, a conjugação deve levar ponto crítico em ponto crítico. Para que a conjugação seja pelo menos $C^{1}$ precisamos que este mapa preserve os graus de cada ponto crítico, pois isto é equivalente a pedir que o raio das medidas dos segmentos limitados por pontos críticos sejam os mesmos para os mapas multicríticos, veja Lema 2.2.4. Este último é um invariante da conjugação $C^{1}$. Um problema interessante seria encontrar todos os invariantes por conjugações suaves, veja [dM11].

Pergunta 5.0.1. Quais são todos os invariantes por conjugaçôes suaves entre mapas multicríticos do círculo?

Conhecer a distância entre as renormalizaçôes geradas pelos dois mapas também permitira obter informação sob a conjugação, como no caso uni-crítico (veja por exemplo, [dFdM99] ou [KT0]). Baseados nesses resultados, estabelecemos a seguinte pergunta no cenário multicrítico:

Pergunta 5.0.2. Sejam $f$ e $g$ dois mapas multicríticos do círculo com mesma assinatura. Se as $n$ ésimas renormalizaçôes ao redor de cada ponto crítico convergem juntamente na topologia $C^{0}$ e exponencialmente rápido, então toda conjugação canônica $h: S^{1} \rightarrow S^{1}$ é um difeomorfismo de classe $C^{1}$. Se o número de rotação é de tipo limitado então $h$ é um difeomorfismo $C^{1+\alpha}$, para $0<\alpha<1$ universal?.

Observação 5.0.1. A pergunta anterior está enunciada para mapas multicríticos com criticalidades impares, pois para o caso uni-crítico isto é valido. Não temos como acreditar ou desacreditar na resposta afirmativa de uma pergunta similar para criticalidades não inteiras, já que para o caso uni-crítico esta problema ainda esta aberto.

Nossa definição do operador renormalização é aquela dada no capítulo 2. Nós acreditamos que este operador é adequado para encarar o problema da suavidade da conjugação. Tal como acontece no caso uni-crítico, para provar a convergência exponencial das renormalizaçôes precisaríamos de um conceito que permita estender o pares multicríticos comutativos (veja capítulo 2 secção 2.3) em todo o plano, isto seria uma especie de pares multicríticos holomorfos (veja [dF99] para a definição de este tipo de mapas no caso uni-crítico e criticalidade impar igual a 3).

No casso uni-crítico, assumindo convergência exponencial das renormalizaçôes podemos achar contraexemplo para a rigidez, veja [dFdM99] e [Avi13]. É natural perguntar-se pela condição mínima que o número de rotação deve satisfazer para garantir a existência de tais contraexemplos. Enunciamos a seguinte conjectura, ainda em aberto, devida a Avila em [Avi13]. 
Conjectura 5.0.1. Se as renormalizações parabólicas de dois mapas uni-críticos do círculo, analítico reais, convergem exponencialmente e se os coeficientes na expansão em fracão contínua não satisfazem $\ln a_{n}=O(n)$, então tais mapas do círculo não são conjugados por um difeomorfismo $C^{1+\alpha}$, para todo $0<\alpha<1$.

A respeito da conjectura 5.0.2, é razonável acreditar que o resultado possa falhar para qualquer número de rotação irracional, tal como acontece no caso uni-crítico. Assim, é de esperar existência de contraexemplos como aqueles construídos em [dFdM99], no caso $C^{\infty}$, e em [Avi13] para o caso real analítico, para que a conjugação não seja $C^{1+\alpha}$.

Seja $\mathbb{A} \subset[0,1]$ o conjunto dos números de rotação $\rho=\left[a_{0}, a_{1}, \cdots\right]$ que satisfazem as seguintes propriedades:

1. $\lim \sup _{n \rightarrow \infty} \frac{1}{n} \sum_{j=1}^{n} \log a_{j}<\infty$,

2. $\lim _{n \rightarrow \infty} \log a_{n}=0$,

3. $\frac{1}{n} \sum_{j=k+1}^{k+n} \log a_{j} \leq \omega_{\rho}\left(\frac{n}{k}\right)$, para todo $0<n \leq k$, onde $\omega_{\rho}$ é uma função que depende unicamente do número de rotaçấo e satisfaz $\omega_{\rho}(t)>0$ para todo $t>0$ e $t \omega_{\rho}(t) \rightarrow 0$ quando $t \rightarrow 0$.

O conjunto $\mathbb{A}$ foi definido em [dFdM99]. Este conjunto tem medida de Lebesgue total e contém o conjunto dos números de tipo limitado, alguns números Liouville e alguns números Diofantinos.

Com a definição do conjunto $\mathbb{A}$, enunciamos mais uma pergunta referente a mapas multicríticos do círculo.

Pergunta 5.0.3. Existem mapas multicríticos do círculo com mesma assinatura e mesmo número de rotação (que não pertence ao conjunto $\mathbb{A}$ ) tal que toda conjugação canônica entre $f$ e g, que preserva os graus de criticalidade, não pode ser um difeomorfismo $C^{1+\alpha}$ para todo $0<\alpha<1$.

Em [Pal15], Palmisano provou dois mapas do círculo com um intervalo flat, mesmo número de rotação de tipo limitado e que satisfazem a consequência do teorema das real bounds para alguma partição do círculo, são conjugados por um mapa quasi-simétrico do círculo. Assim, surge a seguinte pergunta

Pergunta 5.0.4. Dados dois mapas do círculo que possuem um intervalo flat e o mesmo número finito de pontos críticos non-flat, com mesmo número de rotação de tipo limitado. Existe uma conjugação entre estes dois mapas que seja quase-simétrica?. 


\section{Apêndice A}

\section{Mapas do círculo}

Neste apêndice apresentamos os conceitos e técnicas mais relevantes em relação aos mapas do círculo. Além disso, mostramos as provas dos resultados mais relevantes enunciados no capitulo 2 e usados ao longo do texto. A principais referências relacionada com esta parte da teoria são os livros [KH] $e$ [dMvS93].

\section{A.1 Número de rotação}

Dado um homeomorfismo do círculo $f: S^{1} \rightarrow S^{1}$, dizemos que $F: \mathbb{R} \rightarrow \mathbb{R}$ é um levantamento de $f$ se $\pi \circ F=f \circ \pi$, onde $\pi: \mathbb{R} \rightarrow S^{1}$ é uma aplicaçấo de recobrimento, por exemplo $\pi(x)=e^{2 \pi i x}$. $O$ número de rotação do mapa $f$ é o número real definido como

$$
\rho(f)=\rho=\lim _{n \rightarrow \infty} \frac{F^{n}(x)}{n} .
$$

Este limite sempre existe, é independente do ponto $x$ e, se $F_{1}, F_{2}$ são dois levantamentos de $f$ que produzem dois números de rotação $\rho_{1}, \rho_{2} \in \mathbb{R}$ então $\rho_{1}-\rho_{2} \in \mathbb{Z}$. O termo número de rotação foi introduzido pelo Poincaré, [Poi82], esta quantidade, a grosso modo, mede a taxa média de rotação de órbitas ao redor do círculo.

O número de rotação pode ser expresso como uma fração contínua,

$$
\rho=\left[a_{0}, a_{1}, \cdots, a_{n}, \cdots\right]=\frac{1}{a_{0}+\frac{1}{a_{1}+\frac{1}{\frac{\ddots}{a_{n}+\frac{1}{\ddots}}}}}
$$

a qual é finita ou infinita, dependendo de se $\rho$ é racional ou irracional, respectivamente. Truncando

a fração contínua (A.1.1) no nível $n-1$, obtemos a sequência de fraçôes reduzidas $\left\{p_{n} / q_{n}\right\}_{n \in \mathbb{N}}$, os 
convergentes de $\rho$, mais precisamente,

$$
\frac{p_{n}}{q_{n}}=\left[a_{0}, a_{1}, \cdots, a_{n-1}\right]=\frac{1}{a_{0}+\frac{1}{a_{1}+\frac{1}{\ddots \frac{1}{a_{n-1}}}}} .
$$

Cada fração $p_{n} / q_{n}$ é a melhor aproximação a $\rho$ por frações cujo denominador é no máximo $q_{n}$ [Khi97, Capítulo II, Teorema 15], temos:

$$
\text { Se } \quad 0<q<q_{n} \quad \text { então } \quad\left|\rho-\frac{p_{n}}{q_{n}}\right|<\left|\rho-\frac{p}{q}\right|, \quad \text { para todo } p \in \mathbb{N} \text {. }
$$

Definindo $p_{0}=0$ e $p_{1}=1$, temos que a sequência de numeradores satisfaz

$$
p_{n+1}=a_{n} p_{n}+p_{n-1} \quad \text { para } n \geq 1 \text {. }
$$

Analogamente, definindo $q_{0}=1$ e $q_{1}=a_{0}$, obtemos a seguinte propriedade recursiva dos denominadores, os quais chamamos de tempos de retorno,

$$
q_{n+1}=a_{n} q_{n}+q_{n-1} \quad \text { para } n \geq 1
$$

As sequências $\left\{p_{n}\right\}_{n \in \mathbb{N}},\left\{q_{n}\right\}_{n \in \mathbb{N}}$ satisfazem a seguinte propriedade.

Lema A.1.1. [Khig7] Para todo $n \in \mathbb{N}$,

$$
q_{n} p_{n+1}-q_{n+1} p_{n}=(-1)^{n}
$$

Prova do Lema A.1.1. A prova segue-se por indução. Notemos que $q_{0} p_{1}-q_{1} p_{0}=1$ e que, $q_{1} p_{2}-q_{2} p_{1}=$ $a_{0} a_{1}-a_{1} a_{0}-1=-1$. Suponhamos agora que $q_{n} p_{n+1}-q_{n+1} p_{n}=(-1)^{n}$,

$$
\begin{aligned}
q_{n+1} p_{n+2}-q_{n+2} p_{n+1} & =q_{n+1}\left(a_{n+1} p_{n+1}+p_{n}\right)-\left(a_{n+1} q_{n+1}+q_{n}\right) p_{n+1} \\
& =a_{n+1} q_{n+1} p_{n+1}+p_{n} q_{n+1}-a_{n+1} q_{n+1} p_{n+1}-q_{n} p_{n+1} \\
& =-\left(q_{n} p_{n+1}-q_{n+1} p_{n}\right)=(-1)^{n+1}
\end{aligned}
$$

Definição A.1.1. Seja $\rho=\left[a_{0}, a_{1}, \cdots\right] \in \mathbb{R} \backslash \mathbb{Q}$. Se existe $M>0$ tal que $a_{n} \leq M$ para todo $n \in \mathbb{N}$ dizemos que $\rho$ é de tipo limitado.

Exemplo A.1.1 (Proporção áurea). Seja $\rho=\frac{1+\sqrt{5}}{2}$, a proporção áurea ou o número áureo. Os quocientes parciais na fracção continua de $\rho$, são todos iguais a um, i.e. $a_{n}=1$ para todo $n \geq 0$. Pelas propriedades indutivas dos convergentes de $\rho$, obtemos que:

$$
\begin{aligned}
& p_{0}=0, p_{1}=1, p_{2}=1, p_{3}=2, p_{4}=3, p_{5}=5, \cdots \\
& q_{0}=1, q_{1}=1, q_{2}=2, q_{3}=3, q_{4}=5, q_{5}=8, \cdots .
\end{aligned}
$$

Assim, seus convergentes são os raios dos números de Fibonacci.

Se $\beta \geq 0$, o número de rotação $\rho \in \mathbb{R} \backslash \mathbb{Q}$ satisfaz a condição Diophantina da ordem $\beta$ se existe 
uma constante $C>0$ tal que, para todo $p / q \in \mathbb{Q}$

$$
\left|\rho-\frac{p}{q}\right| \geq \frac{C}{q^{2+\beta}} .
$$

Os números que satisfazem (A.1.3) quando $\beta=0$, são os números de tipo limitado. O conjunto dos números de tipo limitado são densos em [0,1] mas tem medida de Lebesgue zero (veja [Khi97, capítulo III, Teorema 29] ou [Gua12, Lema A.1.3]).

Os números Diofantinos são todos os números $\rho$ que satisfazem a desigualdade (A.1.3) para algum $\beta \geq 0$. Este conjunto de números possui medida total de Lebesgue. Todo número irracional que não é Diofantino é chamado número de Liouville, portanto os números de Liouville possuem medida de Lebesgue zero.

Seja $R_{\rho}: S^{1} \rightarrow S^{1}$ a rotação de ângulo $\rho \in \mathbb{R} \backslash \mathbb{Q}$ no círculo, i.e. para todo $x \in S^{1}, R_{\rho}(x)=x+\rho$. Pelas propriedades anteriores, para todo $x \in S^{1}$ e para todo $n \in \mathbb{N}$ os iterados $R_{\rho}^{q_{n}}$ são os retornos mais próximos de $x$, assim para todo $j<q_{n}$,

$$
\left|x-R_{\rho}^{q_{n}}(x)\right|<\left|x-R_{\rho}^{j}(x)\right| .
$$

De fato, os primeiros retornos $q_{n}$ se aproximam ao ponto $x$ de forma alternada:

$$
R_{\rho}^{q_{1}}(x)<R_{\rho}^{q_{3}}(x)<\cdots R_{\rho}^{q_{2 k+1}}(x)<\cdots<x<\cdots<R_{\rho}^{q_{2 k}}(x)<\cdots<R_{\rho}^{q_{2}}(x)<R_{\rho}^{q_{0}}(x) .
$$

O número de rotação é invariante por conjugações ${ }^{1}$ (veja [KH, Capítulo 11, página 388]). A racionalidade ou irracionalidade do número de rotação determina o comportamento do mapa:

Proposição A.1.1. Seja $f$ homeomorfismo do círculo que preserva orientação. Então $\rho(f) \in \mathbb{Q}$ se e somente se f tem uma órbita periódica. Neste caso, todas as órbitas tem o mesmo período.

Veja uma prova da proposição anterior em [KH, Capítulo 11, páginas 389-390]). Em particular, as rotaçôes racionais $R_{p / q}$ satisfazem $R_{p / q}^{n q}(x)=x$, para todo $x \in S^{1}$ e todo $n \in \mathbb{Z}$.

Para o caso irracional temos o seguinte resultado,

Teorema A.1.1 (Teorema de classificação de Poincaré). Seja $f: S^{1} \rightarrow S^{1}$ homeomorfismo que preserva orientação com número de rotação irracional $\rho(f)$.

- Se $f$ é transitivo então $f$ é conjugado à rotação rígida $R_{\rho(f)}$.

- Se $f$ não é transitivo então $f$ é semi-conjugado à rotação $R_{\rho(f)}$.

Veja uma prova do Teorema A.1.1 em [KH, Capítulo 11, páginas 397-398].

Proposição A.1.2. Seja $f$ um homeomorfismo do círculo sem pontos periódicos, então existe um conjunto $K$ tal que $\alpha(x)=\omega(x)=K$, para todo $x \in S^{1}$. Se $K$ tem pontos interiores então $K=S^{1}$, em caso contrário, $K$ é um conjunto de Cantor.

\footnotetext{
${ }^{1}$ Uma conjugação $h: S^{1} \rightarrow S^{1}$ entre dois mapas do círculo $f$ e $g$ é um homeomorfismo que satisfaz $h \circ f=g \circ h$. Neste caso, dizemos que $f$ e $g$ são topologicamente equivalentes

${ }^{2}$ Uma semi-conjugação $h: S^{1} \rightarrow S^{1}$ entre $f$ e $R_{\rho(f)}$ é uma aplicação contínua e sobrejetora tal que $h \circ f=R_{\rho(f)} \circ h$. Neste caso, dizemos que $f$ e $R_{\rho(f)}$ são combinatoriamente equivalentes
} 
Veja [dMvS93, Capítulo páginas 33-34]. O conjunto $K$ da proposição anterior é chamado conjunto minimal de $f$.

Seja $M$ um espaço métrico compacto e $f: M \rightarrow M$ uma aplicação contínua. Dizemos que $f$ é unicamente ergódica se existe uma única medida de probabilidade $\mu$ em $S^{1}$ tal que, para todo $A \subseteq S^{1}$ Boreliano, temos $\mu(A)=\mu\left(f^{-1}(A)\right)$. Lembramos a seguinte propriedade que é satisfeita por rotações irracionais.

Teorema A.1.2. [Teorema da Equidistribuição de Kronecker-Weyl] Toda rotação irracional, $R_{\rho}$, é unicamente ergódica.

Prova do Teorema A.1.2. Nesta prova, vamos usar a notação multiplicativa de $S^{1}$ e o seguinte resultado:

Lema A.1.2. Se para cada função contínua $\phi$, de um conjunto denso no espaço $C(X)$, as médias de tempo $(1 / n) \sum_{k=0}^{n-1} \phi\left(f^{k}(x)\right)$ convergem uniformemente para uma constante então $f$ é unicamente ergódica.

Pelo lema anterior, é suficiente verificar que as médias de tempo para cada função contínua de um conjunto denso de funções uniformemente contínuas convergem para uma constante. Agora, pelo teorema de Weierstrass, os polinômios trigonométricos formam um conjunto denso no conjunto das funções contínuas na topologia uniforme. Como a convergência uniforme a uma constante é uma propriedade linear, então é suficiente verificar convergência uniforme para todo sistema completo de funções, por exemplo, para as funções características $\chi_{m}(x)=\exp (2 \pi i m x)$. Para $m \neq 0$, temos

$$
\chi_{m}\left(R_{\rho}\right)(x)=\exp (2 \pi i m(x+\rho))=\exp (2 \pi i m \rho) \chi_{m}(x)
$$

e também

$$
\left|\frac{1}{n} \sum_{k=0}^{n-1} \chi_{m}\left(R_{\rho}^{k}(x)\right)\right|=\left|\frac{1}{n} \sum_{k=0}^{n-1} \exp (2 \pi i m k \rho)\right|=\frac{|1-\exp (2 \pi i m n \rho)|}{n|\exp (2 \pi i m \rho)|} \leq \frac{2}{n|\exp (2 \pi i m \rho)|} .
$$

Esta ultima expressão vai para zero quando $n \rightarrow \infty$, isto implica que a rotação irracional é unicamente ergódica.

Pelo teorema anterior e Teorema A.1.1, todo homeomorfismo do círculo com número de rotação irracional é unicamente ergódico.

Seja $f$ é um mapa do círculo combinatoriamente equivalente à rotação rígida correspondente. Aqui, $I_{n}(x)$ denota o intervalo do círculo com pontos extremais $x$ e $f^{q_{n}}(x)$. Temos o seguinte resultado:

Lema A.1.3. Para todo $n \geq 0$ e todo $x \in S^{1}$, a coleção de intervalos

$$
\mathcal{P}_{n}(x)=\left\{f^{i}\left(I_{n}(x)\right): 0 \leq i \leq q_{n+1}-1\right\} \cup\left\{f^{j}\left(I_{n+1}(x)\right): 0 \leq j \leq q_{n}-1\right\}
$$

é uma partição do círculo (modulo pontos extremais), chamada a n-ésima partição dinâmica associada ao ponto $x$.

Prova do Lema A.1.3. Por facilidade, nesta prova vamos omitir a letra $x$ em $\mathcal{P}_{n}(x)$. Como as famílias $\mathcal{P}_{n}$ estão dinâmicamente definidas, vamos assumir nesta prova que $f$ é uma rotação rígida no círculo unitário de ângulo $\theta$, onde $\theta \in[0,1)$ é um número irracional.

Sendo irracional, $\theta$ tem uma expansão em fração contínua, digamos $\theta=\left[a_{0}, a_{1}, \cdots\right]$. As propriedades aritméticas da expansão em fração contínua descritas no começo deste apêndice implicam que, para 
todo ponto $x \in S^{1}$, os iterados $\left\{f^{q_{n}}(x)\right\}_{n \in \mathbb{N}}$ são os retornos mais próximos da órbita de $x$ pela rotação rígida $f$, no seguinte sentido:

$$
d\left(x, f^{q_{n}}(x)\right)<d\left(x, f^{j}(x)\right) \quad \text { para todo } j \in\left\{1, \ldots, q_{n}-1\right\}
$$

onde, $d$ denota a distância estândar em $S^{1}$. Em particular, todos os membros da família

$$
\left\{I_{n}, f\left(I_{n}\right), \cdots, f^{q_{n+1}-1}\left(I_{n}\right)\right\}
$$

são dois-a-dois disjuntos, e todos os membros da família

$$
\left\{I_{n+1}, f\left(I_{n+1}\right), \cdots, f^{q_{n}-1}\left(I_{n+1}\right)\right\}
$$

são, também, dois-a-dois disjuntos. Além disso, afirmamos que dois membros qualquer na união de estas famílias (lembre que esta união é precisamente a definição de $\mathcal{P}_{n}$ ) são disjuntos. De fato, suponhamos por contradição que existe $i<q_{n+1}$ e $j<q_{n}$ tal que $f^{i}\left(I_{n}\right) \cap f^{j}\left(I_{n+1}\right) \neq \varnothing$. Sem perdida de generalidade, podemos supor que $i<j=i+l$, para algum $l<q_{n}$, e que o $q_{n}$-ésimo iterado de todo ponto $x \in S^{1}$ esta à esquerda de $x$, portanto o $q_{n+1}$-ésimo iterado esta à direita de $x$. Temos três opções:

(1) Se $f^{j}\left(I_{n+1}\right) \subseteq f^{i}\left(I_{n}\right)$, então o ponto extremal $f^{j}(c)=f^{i+l}(c)$ esta mais próximo a $f^{i}(c)$ que $f^{i+q_{n}}(c)$, mas isto é impossível pois $l<q_{n}$.

(2) Se $f^{i}\left(I_{n}\right) \subseteq f^{j}\left(I_{n+1}\right)$ então $f^{j}\left(I_{n+1}\right)$ intercepta $f^{i}\left(I_{n+1}\right)$ e isto é impossível como tínhamos explicado antes.

(3) Se $f^{j}\left(I_{n+1}\right) \backslash f^{i}\left(I_{n}\right) \neq \varnothing$ então temos duas opções, ou $f^{j}(c) \in f^{i}\left(I_{n}\right)$ ou então $f^{j+q_{n+1}}(c) \in f^{i}\left(I_{n}\right)$. No primeiro caso, o ponto $f^{j}(c)=f^{i+l}(c)$ esta mais próximo a $f^{i}(c)$ que $f^{i+q_{n}}(c)$, e dado que $l<q_{n}$ isto é uma contradição. No segundo caso, o ponto $f^{i+q_{n}}(c)=f^{j}\left(f^{q_{n}+i-j}(c)\right)$ é um ponto que esta mais perto de $f^{j}(c)$ que $f^{j+q_{n+1}}(c)$, isto é impossível pois $q_{n}+i-j<q_{n+1}$.

Portanto, dois membros qualquer de $\mathcal{P}_{n}$ são disjuntos, como tínhamos afirmado.

Finalmente, dado que estamos supondo que $f$ é a rotação rígida de ângulo $\theta$ no círculo unitário (normalizado) os comprimentos dos intervalos $I_{n}$ e $I_{n+1}$ são $\left|q_{n} \theta-p_{n}\right|=q_{n}\left|\theta-p_{n} / q_{n}\right|$ e $q_{n+1} \mid p_{n+1} / q_{n+1}-$ $\theta \mid$, respectivamente. Logo, o comprimento total da união dos membros de $\mathcal{P}_{n}$ é igual a:

$$
\left|q_{n} q_{n+1}\left(\frac{p_{n+1}}{q_{n+1}}-\frac{p_{n}}{q_{n}}\right)\right|=\left|q_{n} p_{n+1}-p_{n} q_{n+1}\right| .
$$

Pelo Lema A.1.1, este valor absoluto é igual a 1, isto é, a união de todos os membros de $\mathcal{P}_{n}$ é um conjunto compacto de medida total de Lebesgue, e assim cobre o círculo inteiro.

\section{A.2 Cross-ratio e derivada Schwarziana}

Seja $N$ o círculo unitário ou o intervalo [0,1]. Sejam $M$ e $T$ dois intervalos em $N$ tal que $M$ esta compactamente contido em $T$ (propriedade que denotamos por $M \Subset T$ ). Definimos o a-cross-ratio $e$ o $b$-cross-ratio do par de intervalos $(M, T)$, respectivamente como segue

$$
a(M, T)=\frac{|M||T|}{|L||R|}, \quad b(M, T)=\frac{|L||R|}{|M \cup L||M \cup R|},
$$

onde $L$ e $R$ são as duas componentes conexas de $T \backslash M$. 
Estes tipos de cross-ratios não são os únicos definimos em dinâmica uni-dimensional, o uso de um ou outro cross-ratio depende dos propósitos dos autores. De fato, a relação entre o a-cross-ratio e o b-cross-ratio esta dada por: $a(M, T)+1=b(M, T)^{-1}$. Notemos que os dois cross-ratios são preservados por mapas Moebius; i.e. se $\phi$ é um mapa Moebius então

$$
a(M, T)=a(\phi(M), \phi(T)) \quad e \quad b(M, T)=b(\phi(M), \phi(T)) .
$$

Neste apêndice, vamos fazer uso do b-cross-ratio, a razão de esta escolha é simplesmente por facilidade de nossas contas. Este cross-ratio esta relacionado com o Modelo de Poincaré do intervalo. Mais precisamente, se $T=(\alpha, \delta)$ é um intervalo de $N$, definimos a densidade de Poincaré de $T$ como

$$
\rho_{T}(x)=\frac{\delta-\alpha}{(x-\alpha)(\delta-x)}
$$

para todo $x \in T$.

Integrando $\rho_{T}(x)$ com respeito de $x$, obtemos a medida de Poincaré em T. Assim, o comprimento de Poincaré do intervalo $M$ (com pontos extremais $x$ e $\alpha+\delta / 2$ ), em $T$ esta dado por

$$
\ell_{T}(M)=\int_{M} \rho_{T}(x) d x=\log b(M, T) .
$$

Existe um conceito que mostra como é alterado qualquer cross-ratio sob homeomorfismos. Seja $f: N \rightarrow N$ uma função continua, e seja $U \subseteq N$ um conjunto aberto tal que $\left.f\right|_{U}$ é um homeomorfismo sob sua imagem. Se $M \subset T \subset U$ são intervalos, com $M \Subset T$, definimos a distorção do b-cross-ratio de f no par $(M, T)$ como o quociente

$$
\mathrm{CrD}(f ; M, T)=\frac{b(f(M), f(T))}{b(M, T)} .
$$

Se $f \mid T$ é uma transformação de Moebius, então $\operatorname{CrD}(f ; M, T)=1$ para todo $M \Subset T$. Além disso, se $\left.f\right|_{T}$ é um difeomorfismo sob sua imagem e $\left.\log D f\right|_{T}$ tem variação limitada $V(f)$ (por exemplo, se $f$ é um difeomorfismo de classe $\left.C^{2}\right)$, então $\operatorname{CrD}(f ; M, T) \leq e^{2 V(f)}$.

Observemos que para $j \geq 1$ temos a seguinte regra da cadeia da distorção de cross-ratios,

$$
\mathrm{CrD}\left(f^{j} ; M, T\right)=\prod_{i=0}^{j-1} \operatorname{CrD}\left(f ; f^{i}(M), f^{i}(T)\right) .
$$

Seja $f: T \rightarrow f(T)$ um difeomorfismo de classe $C^{1}$, definimos a distorção de $f$ como

$$
\operatorname{Dist}(f, T)=\sup _{x, y \in T} \frac{|D f(x)|}{|D f(y)|} .
$$

Notemos que $\operatorname{Dist}(f, T)=1$ se e somente se, $f$ é um mapa afim em $T$. Em qualquer outro caso temos que $\operatorname{Dist}(f, T)>1$. Pelo Teorema do valor Médio, obtemos o seguinte fato

Observação A.2.1. Seja $\varepsilon>0$. Se $\operatorname{Dist}(f, T)<1+\varepsilon$, então $\operatorname{CrD}(f ; M, T)<(1+\varepsilon)^{2}$ para todo $M \subset T \subset N$.

Como foi dito antes, dados dois intervalos $M \subset T \subset S^{1}$ com $M \Subset T$, denotamos por $L$ e $R$ as duas componentes conexas da diferença $T \backslash M$. Definimos o espaço de $M$ em $T$ como o menor dos raios 
$|L| /|M|$ e $|R| /|M|$. Se o espaço é $\tau>0$ dizemos que $T$ contém uma vizinhança $\tau$-escalada of $M$.

Lema A.2.1 (Principio de distorção de Koebe). Para cada $\ell, \tau>0$ e cada homeomorfismo unidimensional $f$ existe uma constante $K=K(\ell, \tau, f)>1$ da forma

$$
K=\left(1+\frac{1}{\tau}\right)^{2} \exp \left(C_{0} \ell\right)
$$

onde $C_{0}$ é uma constante que depende só de $f$, com a seguinte propriedade. Se $T$ é um intervalo tal que $\left.f^{k}\right|_{T}$ é um difeomorfismo sob sua imagem e se $\sum_{j=0}^{j=k}\left|f^{j}(T)\right| \leq \ell$, então para cada intervalo $M \subset T$ para a qual $f^{k}(T)$ contém uma vizinhança $\tau$-escalada de $f^{k}(M)$ temos que,

$$
\frac{1}{K} \leq \frac{\left|D f^{k}(x)\right|}{\left|D f^{k}(y)\right|} \leq K
$$

para todo $x, y \in M$.

A prova deste lema pode ser achada em [dMvS93, Capítulo IV, página 295].

Outra ferramenta usada em dinâmica unidimensional é a derivada Schwarziana. Este operador foi introduzido pela primeira vez por J. L. Lagrange em [Lag79], e deve seu nome ao matemático inglês Arthur Caley, veja [dSG10, Capítulo IV.2, página 149].

Definição A.2.1. Para todo mapa $f: N \rightarrow N$ de classe $C^{3}$ e para todo $x \in N$, ponto regular de $f$, definimos a Derivada Schwarziana de $f$ no ponto $x$ como

$$
S f(x)=\frac{D^{3} f(x)}{D f(x)}-\frac{3}{2}\left(\frac{D^{2} f(x)}{D f(x)}\right)^{2} .
$$

Da definição A.2.1, obtemos a seguinte regra da cadeia

$$
S(g \circ f)(x)=S g(f(x))|D f(x)|^{2}+S f(x),
$$

e para $n \geq 1$,

$$
S f^{n}(x)=\sum_{i=0}^{n-1} S f\left(f^{i}(x)\right)\left|D f^{i}(x)\right|^{2} .
$$

As seguintes são propriedades da derivada Schwarziana de um mapa.

1. $S f=0$ se e somente se $f(x)=\frac{a x+b}{c x+d}$ para $a, b, c, d \in \mathbb{R}$.

2. Se $S f<0$ entâo $S f^{n}<0$, para todo $n \in \mathbb{N}$.

3. (Principio do Minimo) Seja $T=[a, b]$ intervalo fechado e seja $f: T \rightarrow \mathbb{R}$ uma função contínua sem pontos críticos tal que $S f(x)<0$ para todo $x \in T$. Então

$$
|D f(x)|>\min \{|D f(a)|,|D f(b)|\} \quad \text { para todo } x \in(a, b) .
$$

Uma prova destas propriedades pode ser achada em [dMvS93, Capítulo II, seção 6] e [dMvS93, Capitulo IV, seção 1]. 
A relação entre a distorção de cross-ratio e a derivada Schwarziana esta descrita no seguinte resultado

Lema A.2.2. Se $f: N \rightarrow N$ é um difeomorfismo de classe $C^{3}$ com $S f<0$ em todos seus pontos regulares, então para todo par de intervalos $M \subset T \subseteq N$ temos que $\operatorname{CrD}(f ; M, T)<1$.

O lema prévio é um resultado conhecido, mas como ele é usado fortemente neste texto a seguir damos uma prova.

Prova do Lema A.2.2. A prova é uma adaptação de [dMvS93, Seção IV.1] para o b-cross ratio, que é nosso contexto. Primeiro observe que se $\phi$ é uma transformação de Moebius, então $D(\phi, M, T)=1$. Sejam $a, d$ os pontos extremais do intervalo $T$ e $b, c$ os pontos extremos do intervalo $M$. Vamos chamar $R$ e $L$ as duas componentes conexas de $T \backslash M$. Seja $\phi$ a transformação de Moebius tal que $\phi \circ f(a)=a$, $\phi \circ f(b)=b$ e $\phi \circ f(d)=d$. Afirmamos que $\phi \circ f(c)>c$. Suponhamos que não é verdade, isto é, suponhamos que $\phi \circ f(c) \leq c$. Pelo Teorema do Valor Médio existem $z_{0} \in[a, b], z_{1} \in[b, c]$ e $z_{2} \in[c, d]$ tal que

$$
D(\phi \circ f)\left(z_{0}\right)=\frac{\phi(f(a))-\phi(f(b))}{a-b}=1, \quad D(\phi \circ f)\left(z_{1}\right)=\frac{\phi(f(c))-\phi(f(b))}{c-b} \leq 1
$$

$\mathrm{e}$

$$
D(\phi \circ f)\left(z_{2}\right)=\frac{\phi(f(d))-\phi(f(c))}{d-c} \geq 1 .
$$

Se $z_{1} \in\left(z_{0}, z_{2}\right)$, e como $S(\phi \circ f)=S f<0$, as desigualdades anteriores contradizem o principio do mínimo para difeomorfismos do círculo com derivada Schwarziana negativa 3. Portanto, $\phi(f(c))>c$ como tínhamos afirmado. Agora, se $z_{1}=z_{0}$, obtemos que $z_{1}=z_{0}=b$, e então $D(\phi \circ f)(b)=1 \mathrm{e}$ $\phi(f(c))=c$. Isto implica que $D(\phi \circ f)(c)<1$ (de outra forma, o Principio do Mínimo implica que $D(\phi \circ f)(x)>1$ para todo $x \in(b, c)$, o que é impossível pois o mapa $\phi \circ f$ fixa $b$ e $c)$. Novamente, isto contradiz o Principio do Mínimo já que $c \in\left(b, z_{2}\right)$. O caso restante $z_{1}=z_{2}$, é análogo.

A desigualdade $\phi(f(c))>c$, implica que:

$$
\operatorname{CrD}(\phi \circ f ; M, T)=\frac{b(\phi \circ f(M), \phi \circ f(T))}{b(M, T)}=\frac{|M \cup L||\phi(f(c))-d|}{|R||a-\phi(f(c))|}<1 .
$$

E como $\phi$ é Moebius, $\operatorname{CrD}(\phi \circ f ; M, T)=\operatorname{CrD}(f ; M, T)$ e assim temos provado o lema.

Portanto, um mapa com derivada Schwarziana negativa contrai b-cross-ratios. Em contraste, mapas com derivada Schwarziana negativa expandem a-cross-ratios, i.e. para todo $M \Subset T \subseteq N$ temos $a(f(M), f(T))>a(M, T)$ (veja [dMvS93, Capitulo IV, página 273]). De fato, f preserva cross-ratios se e somente se $S f=0$.

\section{A.3 Pontos críticos non-flat}

Definição A.3.1. Seja I um intervalo compacto ou o círculo $S^{1}$, e seja $f: I \rightarrow I$ um mapa $C^{1}$. Seja c um ponto crítico de $f$, i.e. $D f(c)=0$, se para todo $d \in \mathbb{N}$ temos que

$$
\lim _{t \rightarrow 0} \frac{D f(c+t)}{t^{d-1}}=0
$$

dizemos que c é um ponto crítico de $f$ de tipo flat. Isto implica que $f$ é $C^{\infty}$ em c, além disso, a derivada de qualquer ordem de $f$ no punto c é nula. 
Caso contrário, dizemos que c é um ponto crítico de tipo non-flat de ordem d, se d é o menor inteiro tal que:

$$
\lim _{t \rightarrow 0} \frac{D f(c+t)}{t^{d-1}} \neq 0 .
$$

O número d também é chamado de criticalidade ou grau do ponto crítico c. Neste casso, $f$ é de classe $C^{d}$ no ponto crítico e temos que $D^{n} f(c)=0$ para todo $n \in\{1,2, \cdots, d-1\}$ e $D^{d} f(c)=0$. De fato, o valor do limite A.3.1 é $\frac{D^{d} f(c)}{(d-1) !}$.

Se o mapa é suficientemente suave, a definição anterior implica o seguinte resultado

Lema A.3.1. Seja $f$ de classe $C^{r}$ para $r \geq d$. O ponto crítico c é non-flat de ordem $d$ se e somente se existem difeomorfismos locais $\varphi, \psi$ de classe $C^{r} \operatorname{com} \varphi(c)=\psi \circ f(c)=0$ e tal que $\psi \circ f \circ \varphi^{-1}$ é o mapa $t \rightarrow t^{d}$ ao redor de zero.

Prova do Lema A.3.1. Seja $\varphi$ um difeomorfismo do círculo de classe $C^{r} \operatorname{com} \varphi(c)=0$ e denotemos por $g$ o mapa $t \rightarrow t^{d}$. Definimos $\psi=g \circ \varphi \circ f^{-1}$, então $\psi(f(c))=0$. Seja $x$ um ponto numa vizinhança de zero,

$$
D \psi(x)=D \varphi\left(f^{-1}(x)\right) D g\left(\varphi \circ f^{-1}(x)\right) \frac{1}{D f\left(f^{-1}(x)\right)},
$$

tomando limite,

$$
\begin{aligned}
\lim _{x \rightarrow 0} D \psi(x) & =\lim _{x \rightarrow 0} D \varphi\left(f^{-1}(x)\right) D g\left(\varphi \circ f^{-1}(x)\right) \frac{1}{D f\left(f^{-1}(x)\right)} \\
& =\lim _{t \rightarrow 0} D \varphi(c+t) \frac{d(\varphi(c+t))^{d-1}}{D f(c+t)} \\
& =\frac{d ! D \varphi(c)}{D^{d} f(c)} .
\end{aligned}
$$

Portanto, $\psi$ é diferenciável numa vizinhança de zero, e sua inversa, que esta dada por $\psi^{-1}=f \circ \varphi^{-1} \circ g^{-1}$, também é diferenciável.

Observação A.3.1. Se c é um ponto crítico de tipo non-flat de $f$, então existe uma vizinhança $\mathcal{U}$ de c tal que $S f(x)<0$ para todo $x \in \mathcal{U} \backslash\{c\}$.

A seguinte propriedade é válida em torno do ponto crítico de um mapa crítico do círculo. Como esta propriedade é muito usada nesta tese, fornecemos uma prova inspirada na aquela encontrada em [Gua12, Apêndice A].

Corolário A.3.1. Seja $f$ homeomorfismo do círculo de classe $C^{d}$. O ponto crítico $c$ é de tipo non-flat se e somente se para todo $C>1$ existe $\mathcal{U}$ vizinhança de $c$ tal que para todo $t_{0}, t_{1} \in \mathcal{U} \backslash\{c\}$ temos

$$
C^{-1}\left|\frac{t_{0}-c}{t_{1}-c}\right|^{d} \leq \frac{\left|f\left(t_{0}\right)-f(c)\right|}{\left|f\left(t_{1}\right)-f(c)\right|} \leq C\left|\frac{t_{0}-c}{t_{1}-c}\right|^{d} .
$$

Prova do corolário A.3.1. (Veja [Gua12, Apendice A, Lema A.3.1]) Suponhamos que a desigualdade A.3.2 é válida. Pelo Teorema de Taylor, existe $\mathcal{U}$ vizinhança de $c$ tal que para todo $t \in \mathcal{U}$ temos

$$
f(t)-f(c)=\sum_{n=1}^{d} \frac{D^{n} f(c)}{n !}(t-c)^{n}+r(t),
$$


onde $\lim _{t \rightarrow c} \frac{r(t)}{(t-c)^{d}}=0$.

Por hipótese, para $t \in \mathcal{U}$ temos a seguinte cota por cima

$$
\frac{f(t)-f(c)}{|t-c|^{d}}=\sum_{n=1}^{d} \frac{D^{n} f(c)}{n !}(t-c)^{n-d}+\frac{r(t)}{(t-c)^{d}} \leq C \max _{t^{*} \in \mathcal{U}} \frac{\left|f\left(t^{*}\right)-f(c)\right|}{\left|t^{*}-c\right|^{d}} .
$$

Logo, o seguinte limite existe

$$
M:=\lim _{t \rightarrow c} \frac{f(t)-f(c)}{|t-c|^{d}}=\lim _{t \rightarrow c} \sum_{n=1}^{d} \frac{D^{n} f(c)}{n !} \frac{1}{(t-c)^{d-n}}+\lim _{t \rightarrow c} \frac{r(t)}{|t-c|^{d}} .
$$

Como $M$ não é infinito,

$$
\lim _{t \rightarrow c}\left[\frac{D f(c)}{(t-c)^{d-1}}+\frac{D^{2} f(c)}{2 !(t-c)^{d-2}}+\cdots+\frac{D^{d-1} f(c)}{(d-1) !(t-c)}+\frac{D^{d} f(c)}{d !}\right] \neq \infty,
$$

portanto $D f(c)=D^{2} f(c)=\cdots=D^{d-1} f(c)=0$.

Por outro lado, como $M$ não é zero então

$$
\lim _{t \rightarrow c}\left[\frac{D f(c)}{(t-c)^{d-1}}+\frac{D^{2} f(c)}{2 !(t-c)^{d-2}}+\cdots+\frac{D^{d} f(c)}{d !(t-c)^{d}}\right] \neq 0,
$$

e assim, $D^{d} f(c) \neq 0$. Consequentemente, $c$ é um ponto crítico de $f$ de tipo non-flat de ordem $d$. Agora, suponhamos que $c$ é um ponto crítico de ordem $d$. Para $C$ constante maior que um, consideremos a função

$$
\varepsilon(C)=\frac{\left|D^{d} f(c)\right|}{d !} \frac{(C-1)}{(C+1)}
$$

Como $D^{d} f(c) \neq 0$ então a função $\varepsilon$ é um difeomorfismo real-analítico que preserva orientação para valores de $C$ em $(1,+\infty)$ e toma os valores no intervalo $\left(0,\left|D^{d} f(c)\right| / d\right.$ !). A inversa de $\varepsilon(C)$ esta dada por

$$
C(\varepsilon)=\frac{\left|D^{d} f(c)\right|+d ! \varepsilon}{\left|D^{d} f(c)\right|-d ! \varepsilon}
$$

Pelo teorema de Taylor,

$$
\lim _{t \rightarrow c}\left(\frac{|f(t)-f(c)|}{|t-c|^{d}}\right)=\frac{\left|\left(D^{d} f(c)\right)\right|}{d !} \neq 0 .
$$

Para $C>1$ dado e $\varepsilon(C) \in\left(0,\left|D^{d} f(c)\right| / d\right.$ !), seja $\mathcal{U}$ vizinhança de $c$ tal que para todo $t \in \mathcal{U} \backslash\{c\}$ temos:

$$
\frac{\left|D^{d} f(c)\right|}{d !}-\varepsilon \leq \frac{|f(t)-f(c)|}{|t-c|^{d}} \leq \frac{\left|D^{d} f(c)\right|}{d !}+\varepsilon
$$

e assim,

$$
\left(\frac{\left|D^{d} f(c)\right|}{d !}-\varepsilon\right)|t-c|^{d} \leq|f(t)-f(c)| \leq\left(\frac{\left|D^{d} f(c)\right|}{d !}+\varepsilon\right)|t-c|^{d}
$$




\section{Apêndice B}

\section{Prova do Teorema 2.2.2}

Neste apêndice vamos provar o teorema das apriori real bounds (ou Limites Reais) para mapas multicríticos do círculo ou Teorema 2.2.2. A prova que apresentamos aqui é a mesma encontrada em [EdF15], que em sua vez foi inspirada em aquela encontrada no artigo [dFdM99] para o caso de mapas com um único ponto crítico. A diferença entre estas duas provas é que, no caso de um ponto crítico os mapas $f^{i_{2}-i_{1}}: I_{n}^{i_{1}} \rightarrow I_{n}^{i_{2}}$, para $1 \leq i_{1}<i_{2} \leq q_{n+1}$, são difeomorfismos e portanto é possivel usar $o$ Principio de Distorção de Koebe (veja Lema A.2.1 em A) para controlar a distorção do mapa. No caso de mais pontos críticos, não é possivel usar este principio pois os mapas considerados anteriormente não são mais difeomorfismos. Neste caso, usamos a Desigualdade de Cross-Ratio (veja Lema 2.2.2 em capitulo 2).

\section{B.1 Comparabilidade de intervalos dinâmicamente simétricos}

Seguindo o roteiro usado em [dFdM99] vamos provar a desigualdade 2.2.1 para intervalos dinamicamente simétricos, i.e. para intervalos com um ponto extremo em comum $x \in S^{1}$, e os outros pontos extremais dados por $f^{q_{n}}(x)$ e $f^{-q_{n}}(x)$, para algum $n>0$. Para isto vamos precisar dos lemas B.1.1 e B.1.2, embaixo.

Lema B.1.1. Existe uma constante $C_{1}>1$ que depende só de $f$ e satisfaz o seguinte. Para cada $n \geq 0$ existem $z_{1}, z_{2}, z_{3}, z_{4}$ e $z_{5}$ pontos em $S^{1}$ com $z_{j+1}=f^{q_{n}}\left(z_{j}\right)$ tal que

$$
C_{1}^{-1} \leq \frac{\left|z_{i-1}-z_{i}\right|}{\left|z_{i+1}-z_{i}\right|} \leq C_{1}, \quad \text { para } i=2,3,4
$$

Demonstração. Seja $n \geq 0$ fixo e seja $z \in S^{1}$ o ponto tal que $\left|f^{q_{n}}(z)-z\right| \leq\left|f^{q_{n}}(x)-x\right|$, para todo $x \in S^{1}$. Consideremos os seguintes sete pontos

$$
\begin{gathered}
z_{0}=f^{-4 q_{n}}(z), z_{1}=f^{-3 q_{n}}(z), z_{2}=f^{-2 q_{n}}(z), z_{3}=f^{-q_{n}}(z), \\
z_{4}=z, z_{5}=f^{q_{n}}(z), z_{6}=f^{2 q_{n}}(z) .
\end{gathered}
$$

Notemos que pela escolha de $z$,

$$
\left|z_{4}-z_{5}\right| \leq\left|z_{i}-z_{i+1}\right|, \text { para todo } 0 \leq i \leq 5 .
$$

Pela monotonicidade de $f^{q_{n}}$, os sete pontos estão ordenados (em sentido horário ou anti-horário) da forma em que foram introduzidos. Seja $J \subseteq S^{1}$ o intervalo com pontos extremais $z_{0}$ e $z_{6}$ que contém o ponto $z_{4}$. Para $0 \leq i \leq 3$ definimos $T_{i}=\left[z_{i}, z_{i+1}\right] \subseteq J$ e $M_{i}=\left[z_{i+1}, z_{i+2}\right] \subseteq T_{i}$. Então o homeomorfismo 
$f^{q_{n}}$ leva o intervalo $T_{i}$ sob $T_{i+1}$ e $M_{i}$ sob $M_{i+1}$, para $0 \leq i \leq 2$. Além disso, pela forma em que os pontos $z_{i}, \cdots, z_{i+3}$ estão ordenados, a coleção de intervalos $\left\{T_{i}, f\left(T_{i}\right), \ldots, f^{q_{n}}\left(T_{i}\right)\right\}$ tem multiplicidade de intersecção igual a 3.

(i) Vamos provar B.1.1 para $i=4$. Observe que por B.1.2 a desigualdade à esquerda é válida com constante igual a um. Logo, só temos que provar a desigualdade da direita. Aplicando a Desigualdade de Cross-Ratio para o mapa $f^{q_{n}}$ no par $\left(M_{2}, T_{2}\right)$ temos que existe uma constante $B>1$, que depende de $f$ tal que

$$
D\left(f^{q_{n}} ; M_{2}, T_{2}\right)=\frac{b\left(f^{q_{n}}\left(M_{2}\right), f^{q_{n}}\left(T_{2}\right)\right)}{b\left(M_{2}, T_{2}\right)}=\frac{\left|z_{3}-z_{4}\right|\left|z_{5}-z_{6}\right|\left|z_{2}-z_{4}\right|}{\left|z_{4}-z_{6}\right|\left|z_{2}-z_{3}\right|\left|z_{4}-z_{5}\right|} \leq B
$$

ou, equivalentemente, usando $\left|z_{2}-z_{3}\right| \leq\left|z_{2}-z_{4}\right|$

$$
\frac{\left|z_{3}-z_{4}\right|}{\left|z_{4}-z_{5}\right|} \leq B \frac{\left|z_{4}-z_{6}\right|}{\left|z_{5}-z_{6}\right|}=B\left(\frac{\left|z_{4}-z_{5}\right|}{\left|z_{5}-z_{6}\right|}+1\right) \leq 2 B
$$

Portanto, definindo $B_{1}=2 B=\max \{1,2 B\}$ obtemos

$$
B_{1}^{-1} \leq \frac{\left|z_{3}-z_{4}\right|}{\left|z_{4}-z_{5}\right|} \leq B_{1}
$$

(ii) Agora, vamos provar B.1.1 para $i=3$. Usando o item (i) e B.1.2 temos $\left|z_{3}-z_{4}\right| \leq B_{1}\left|z_{2}-z_{3}\right|$. Para obter a outra desigualdade, vamos considerar os intervalos $M_{1}$ e $T_{1}$, e o mapa $f^{q_{n}}$. Pela Desigualdade de Cross-Ratio, obtemos

$$
D\left(f^{q_{n}} ; M_{1}, T_{1}\right)=\frac{b\left(f^{q_{n}}\left(M_{2}\right), f^{q_{n}}\left(T_{2}\right)\right)}{b\left(M_{1}, T_{1}\right)}=\frac{\left|z_{2}-z_{3}\right|\left|z_{4}-z_{5}\right|\left|z_{1}-z_{3}\right|}{\left|z_{3}-z_{5}\right|\left|z_{1}-z_{2}\right|\left|z_{3}-z_{4}\right|} \leq B
$$

Isto é,

$$
\frac{\left|z_{2}-z_{3}\right|}{\left|z_{3}-z_{4}\right|} \leq B \frac{\left|z_{1}-z_{2}\right|\left|z_{3}-z_{5}\right|}{\left|z_{1}-z_{3}\right|\left|z_{4}-z_{5}\right|} \leq B \frac{\left|z_{3}-z_{5}\right|}{\left|z_{4}-z_{5}\right|}
$$

Usando $\left|z_{3}-z_{5}\right|=\left|z_{3}-z_{4}\right|+\left|z_{4}-z_{5}\right|$ e o item $(i)$, temos

$$
\frac{\left|z_{2}-z_{3}\right|}{\left|z_{3}-z_{4}\right|} \leq B\left(\frac{\left|z_{3}-z_{4}\right|}{\left|z_{4}-z_{5}\right|}+1\right) \leq B\left(B_{1}+1\right)
$$

Agora, definindo $B_{2}=B\left(B_{1}+1\right)$ e colocando as duas desigualdades juntas,

$$
B_{2}^{-1} \leq \frac{\left|z_{2}-z_{3}\right|}{\left|z_{3}-z_{4}\right|} \leq B_{2}
$$

(iii) Finalmente, vamos provar B.1.1 para $i=2$. Observemos que o item $(i i)$, item $(i)$ e B.1.2 implicam,

$$
\left|z_{3}-z_{2}\right| \leq B_{2}\left|z_{4}-z_{3}\right| \leq B_{1} B_{2}\left|z_{4}-z_{5}\right| \leq B_{1} B_{2}\left|z_{1}-z_{2}\right| \text {. }
$$

Para provar a outra desigualdade, vamos considerar o mapa $f^{q_{n}}$ e o par de intervalos $\left(M_{0}, T_{0}\right)$. Pela Desigualdade de Cross-Ratio, existe $B>1$ tal que

$$
D\left(f^{q_{n}} ; M_{0}, T_{0}\right)=\frac{b\left(f^{q_{n}}\left(M_{0}\right), f^{q_{n}}\left(T_{0}\right)\right)}{b\left(M_{0}, T_{0}\right)}=\frac{\left|z_{1}-z_{2}\right|\left|z_{3}-z_{4}\right|\left|z_{0}-z_{2}\right|}{\left|z_{2}-z_{3}\right|\left|z_{0}-z_{1}\right|\left|z_{2}-z_{4}\right|} \leq B .
$$


De isto, usando que $\left|z_{0}-z_{1}\right| \leq\left|z_{0}-z_{2}\right|$ e o item (ii) acima, temos

$$
\frac{\left|z_{1}-z_{2}\right|}{\left|z_{2}-z_{3}\right|} \leq B \frac{\left|z_{2}-z_{4}\right|}{\left|z_{3}-z_{4}\right|} \leq B\left(\frac{\left|z_{2}-z_{3}\right|}{\left|z_{3}-z_{4}\right|}+1\right) \leq B\left(B_{2}+1\right)
$$

Definindo $B_{3}=\max \left\{B\left(B_{2}+1\right), B_{2} B_{1}\right\}=B_{1} B_{2}$, obtemos

$$
B_{3}^{-1} \leq \frac{\left|z_{1}-z_{2}\right|}{\left|z_{2}-z_{3}\right|} \leq B_{3}
$$

Temos provado B.1.1 para $C_{1}=\max \left\{B_{1}, B_{2}, B_{3}\right\}=B_{3}>1$, constante que só depende de $f$.

Lema B.1.2. Existe uma constante $C_{2}>1$ que só depende de $f$ e satisfaz o seguinte. Sejam $z_{1}, z_{2}, z_{3}, z_{4}$ e $z_{5}$ pontos dados pelo Lema B.1.1. Se $w_{0}, w_{1}, w_{2}, w_{3}$ e $w_{4}$ são pontos no círculo tal que $w_{j+1}=f^{q_{n}}\left(w_{j}\right)$ e tal que $w_{1}$ pertence no intervalo com pontos extremais $z_{1}$ e $z_{2}$ que não contém o ponto $z_{3}$, então

$$
\frac{\left|w_{1}-w_{2}\right|}{\left|w_{0}-w_{1}\right|} \leq C_{2} \quad \text { e } \quad C_{2}^{-1} \leq \frac{\left|w_{i-1}-w_{i}\right|}{\left|w_{i}-w_{i+1}\right|} \leq C_{2} \quad \text { para } i=2,3 .
$$

Demonstração. Para provar a primeira desigualdade, consideramos o intervalo $T$ com pontos extremos $w_{0}$ e $w_{3}$, contendo os pontos $z_{1}, w_{1}, z_{2}, w_{2}$ e $z_{3}$, e o subintervalo $M=\left[w_{1}, w_{2}\right] \subset T$. Notemos que a coleção $\left\{T, f(T), \ldots, f^{q_{n}}(T)\right\}$ tem multiplicidade de intersecção igual a 3. Aplicando a Desigualdade de Cross-Ratio a $f^{q_{n}}$ e ao par $(M, T)$, obtemos $b\left(f^{q_{n}}(M), f^{q_{n}}(T)\right) \leq B b(M, T)$, ou equivalentemente

$$
\frac{\left|w_{1}-w_{2}\right|\left|w_{3}-w_{4}\right|}{\left|w_{1}-w_{3}\right|\left|w_{2}-w_{4}\right|} \leq B \frac{\left|w_{0}-w_{1}\right|\left|w_{2}-w_{3}\right|}{\left|w_{0}-w_{2}\right|\left|w_{1}-w_{3}\right|}
$$

Como os pontos $w_{0}, z_{1}, w_{1}, \ldots, z_{4}, w_{4}, z_{5}$ estão ciclicamente ordenados como foram dados, temos as desigualdades $\left|z_{1}-z_{2}\right| \leq\left|w_{0}-w_{2}\right|,\left|w_{2}-w_{3}\right| \leq\left|z_{2}-z_{4}\right|$, e $\left|w_{2}-w_{4}\right| \leq\left|z_{2}-z_{5}\right|$. Além disso, pela escolha de $z=z_{4}$ no Lema B.1.1, temos $\left|z_{4}-z_{5}\right| \leq\left|w_{3}-w_{4}\right|$. Usando estos fatos em (B.1.7), obtemos

$$
\frac{\left|w_{1}-w_{2}\right|}{\left|w_{0}-w_{1}\right|} \leq B \frac{\left|z_{2}-z_{4}\right|\left|z_{2}-z_{5}\right|}{\left|z_{1}-z_{2}\right|\left|z_{4}-z_{5}\right|} \leq B\left(C_{1}+C_{1}^{2}\right)\left(1+C_{1}+C_{1}^{2}\right)
$$

onde temos usado as desigualdades do Lema B.1.1.

Para provar a cota superior na última desigualdade em (B.1.6), temos que observar que $\left|w_{i}-w_{i+1}\right| \geq$ $\left|z_{4}-z_{5}\right|$ e que $\left|w_{i-1}-w_{i}\right| \leq\left|z_{i-1}-z_{i+1}\right|$. Usando as desigualdades (B.1.1), deduzimos que

$$
\frac{\left|w_{i-1}-w_{i}\right|}{\left|w_{i}-w_{i+1}\right|} \leq \frac{\left|z_{i-1}-z_{i}\right|}{\left|z_{4}-z_{5}\right|}+\frac{\left|z_{i}-z_{i+1}\right|}{\left|z_{4}-z_{5}\right|} \leq 2 C_{1}^{3}
$$

Para finalizar, a cota inferior da mesma desigualdade em (B.1.6) é obtida da mesma forma (o valor da constante é $\left.\left(2 C_{1}^{3}\right)^{-1}\right)$. Assim, (B.1.6) é verdadeira para $C_{2}=\max \left\{2 C_{1}^{3}, B\left(C_{1}+C_{1}^{2}\right)\left(1+C_{1}+C_{1}^{2}\right)\right\}$.

Com os dois lemas anteriores estamos em condiçôes de provar que intervalos dinâmicamente simétricos são comparáveis. Observamos que, dado $\xi \in S^{1}$, seja $J_{n}(\xi) \subset S^{1}$ o intervalo com pontos extremais $f^{-q_{n}}(\xi)$ e $f^{q_{n}}(\xi)$ que contém $\xi$. Entâo $\bigcup_{i=0}^{q_{n+1}} f^{-i}\left(J_{n}(\xi)\right)=S^{1}$. 
Lema B.1.3 (Intervalos dinâmicamente simétricos são comparáveis). Existe uma constante $C_{3}>1$ que depende só de $f$ tal que, para todo $n \geq 0$ e todo $x \in S^{1}$, temos

$$
C_{3}^{-1}\left|x-f^{-q_{n}}(x)\right| \leq\left|f^{q_{n}}(x)-x\right| \leq C_{3}\left|x-f^{-q_{n}}(x)\right| .
$$

Demonstração. Notemos que é suficiente provar a segunda desigualdade em B.1.10 para todo $x \in S^{1}$ (para obter a primeira, substituímos $x$ por $f^{-q_{n}}(x)$ ).

Seja $x \in S^{1}$, e sejam $z_{1}, z_{2}, \cdots, z_{5}$ os pontos dados pelo Lema B.1.1. Pela observação dada antes do Lema, aplicado a $\xi=z_{2}$ (portanto $J_{n}\left(z_{2}\right)=J$ ), existe $0 \leq i \leq q_{n+1}$ tal que $f^{i}(x)$ pertence ao intervalo $J$ cujos pontos extremais são $z_{1}$ e $z_{3}$ e contém o ponto $z_{2}$. Então temos que $f^{i}(x) \in\left[z_{1}, z_{2}\right] \subset J$, ou $f^{i}(x) \in\left(z_{2}, z_{3}\right] \subset J$. Vamos assumir que estamos no primeiro caso, a prova para o segundo caso é análoga.

Consideramos os pontos $w_{0}=f^{i-q_{n}}(x), w_{1}=f^{i}(x), w_{2}=f^{i+q_{n}}(x)$ e $w_{3}=f^{i+2 q_{n}}(x)$. Seja $T$ o intervalo com pontos extremais $f^{-q_{n}}(x)$ e $f^{2 q_{n}}(x)$ que contém o ponto $x$, e seja $M=\left[x, f^{q_{n}}(x)\right] \subset T$. Observamos que

$$
b(M, T)=\frac{\left|x-f^{-q_{n}}(x)\right|\left|f^{q_{n}}(x)-f^{2 q_{n}}(x)\right|}{\left|f^{q_{n}}(x)-f^{-q_{n}}(x)\right|\left|x-f^{2 q_{n}}(x)\right|} \leq \frac{\left|x-f^{-q_{n}}(x)\right|}{\left|f^{q_{n}}(x)-x\right|} .
$$

Usando as desigualdades B.1.6 no Lema B.1.2, temos

$$
b\left(f^{i}(M), f^{i}(T)\right)=\frac{\left|w_{0}-w_{1}\right|\left|w_{2}-w_{3}\right|}{\left|w_{0}-w_{2}\right|\left|w_{1}-w_{3}\right|} \geq \frac{1}{\left(1+C_{2}\right)^{2}} .
$$

Como $\left\{T, f(T), \ldots, f^{i}(T)\right\}$ tem multiplicidade de intersecção máximo 3, então pela Desigualdade de Cross-Ratio $b\left(f^{i}(M), f^{i}(T)\right) \leq B b(M, T)$, onde o valor da constante $B$ é a mesma dos lemas prévios. Combinando este fato junto com as desigualdades (B.1.11) e (B.1.12), obtemos que

$$
\left|f^{q_{n}}(x)-x\right| \leq B\left(1+C_{2}\right)^{2}\left|x-f^{-q_{n}}(x)\right|
$$

Logo, temos provado B.1.10, para a constante $C_{3}=B\left(1+C_{2}\right)^{2}$.

\section{B.2 Comparabilidade de seis intervalos dinâmicos}

Com a comparabilidade dos intervalos dinamicamente simétricos vamos provar o seguinte resultado, Proposição B.2.1 embaixo. Este resultado afirma que os átomos da partição $\mathcal{P}_{n}$ que estão mais próximos do ponto crítico $c_{0}$, são dois a dois comparáveis.

Proposição B.2.1. Os seis intervalos na Figura B.1 são dois a dois comparáveis. Isto é, existe uma constante $C_{4}>1$ que depende só de $f$ tal que, para todo $n \geq 1$ e para todo $I, J$ intervalos adjacentes da familia $\left\{I_{n}, I_{n+1}, I_{n}^{q_{n}}, I_{n}^{q_{n+1}}, I_{n+1}^{q_{n}}, I_{n}^{q_{n+1}-q_{n}}\right\}$, temos

$$
\frac{1}{C_{4}} \leq \frac{|I|}{|J|} \leq C_{4}
$$

Demonstração. A prova vai ser dividida nos seguintes passos. 


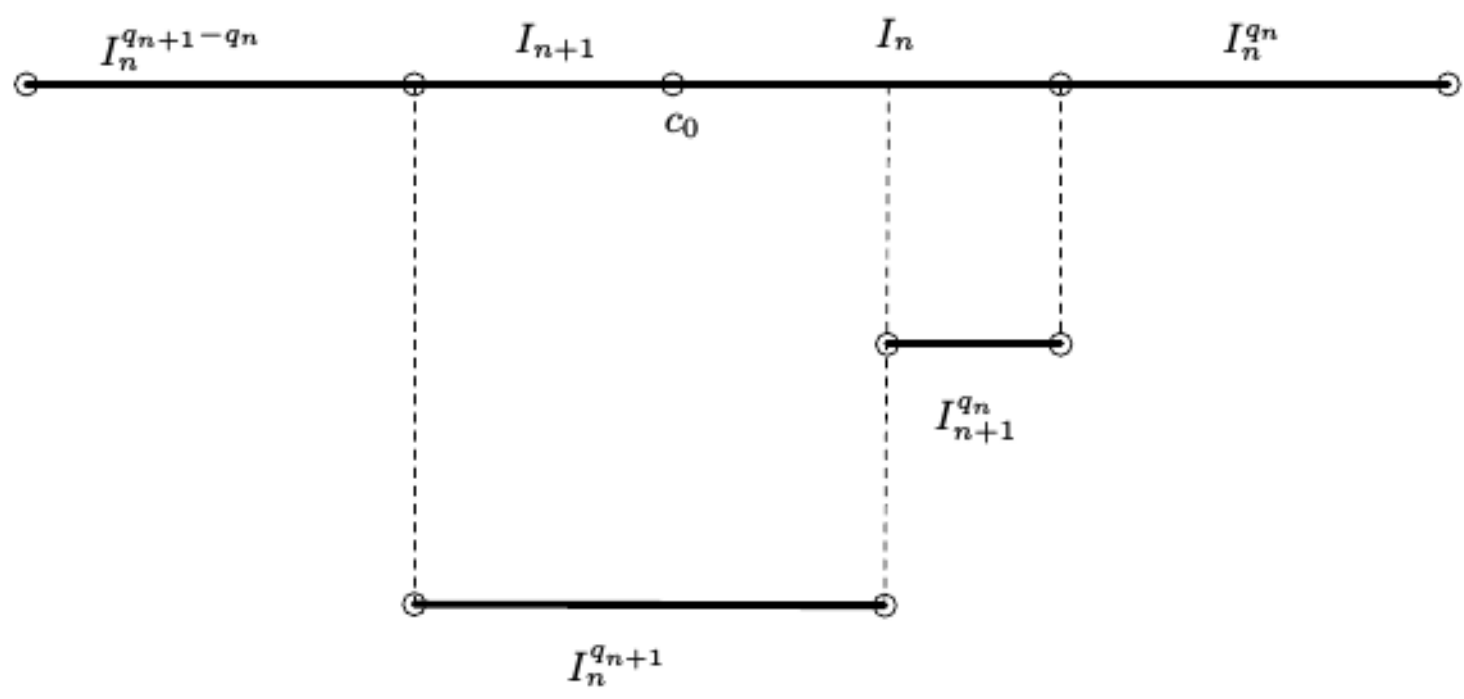

Figura B.1: Seis intervalos comparáveis.

(i) Os intervalos $I_{n}$ e $I_{n}^{q_{n}}$ são comparáveis. Como estes intervalos são dinamicamente simétricos, com respeito a seu ponto extremo em comum $f^{q_{n}}\left(c_{0}\right)$, então pelo Lema B.1.3 temos que

$$
C_{3}^{-1}\left|I_{n}\right| \leq\left|I_{n}^{q_{n}}\right| \leq C_{3}\left|I_{n}\right|
$$

(ii) Os intervalos $I_{n}^{q_{n+1}}$ e $I_{n}^{q_{n+1}-q_{n}}$ são comparáveis. Dado que estes intervalos são dinamicamente simétricos com respeito do ponto extremo $f^{q_{n+1}}\left(c_{0}\right)$, então novamente pelo Lema B.1.3 obtemos

$$
C_{3}^{-1}\left|I_{n}^{q_{n+1}}\right| \leq\left|I_{n}^{q_{n+1}-q_{n}}\right| \leq C_{3}\left|I_{n}^{q_{n+1}}\right| .
$$

(iii) Os intervalos $I_{n}^{q_{n+1}-q_{n}}$ e $I_{n}$ são comparáveis. Consideremos o intervalo $I_{n}^{-q_{n}}$, com pontos extremais $c_{0}$ e $f^{-q_{n}}\left(c_{0}\right)$. Como este intervalo é dinamicamente simétrico com o intervalo $I_{n}$, então pelo Lema B.1.3 temos

$$
C_{3}^{-1}\left|I_{n}^{-q_{n}}\right| \leq\left|I_{n}\right| \leq C_{3}\left|I_{n}^{-q_{n}}\right| .
$$

Do lado direito da desigualdade (B.2.4), a inclusão $I_{n}^{-q_{n}} \subseteq I_{n}^{q_{n+1}-q_{n}} \cup I_{n}^{q_{n+1}}$ e o lado esquerdo da desigualdade (B.2.3), podemos deduzir que

$$
\left|I_{n}\right| \leq C_{3}\left(C_{3}+1\right)\left|I_{n}^{q_{n+1}-q_{n}}\right|
$$

Agora, como $I_{n}^{q_{n+1}} \subseteq I_{n}^{-q_{n}} \cup I_{n}$, e além disso, os intervalos $I_{n}^{-q_{n}}$ e $I_{n}$ são dinamicamente simétricos, então usando a desigualdade da direita de (B.2.3) obtemos

$$
\left|I_{n}^{q_{n+1}-q_{n}}\right| \leq C_{3}\left(C_{3}+1\right)\left|I_{n}\right|
$$

De esta última desigualdade junto com (B.2.5), chegamos a

$$
C_{3}^{-1}\left(C_{3}+1\right)^{-1}\left|I_{n}\right| \leq\left|I_{n}^{q_{n+1}-q_{n}}\right| \leq C_{3}\left(C_{3}+1\right)\left|I_{n}\right|
$$

(iv) Os intervalos $I_{n}$ e $I_{n+1}$ são comparáveis. É aqui que usamos a propriedade de Power-Law do ponto crítico $c_{0}$. Note que $I_{n+1}^{-q_{n+1}} \subseteq I_{n}$ e que os intervalos $I_{n+1}^{-q_{n+1}}$ e $I_{n+1}$ são dinâmicamente simétricos 
com respeito do seu ponto extremal em comum $c_{0}$. Assim, usando o Lema B.1.3 obtemos

$$
\left|I_{n+1}\right| \leq C_{3}\left|I_{n}\right|
$$

Só temos que provar a desigualdade na direção oposta. Consideremos o intervalo $T=I_{n+1} \cup$ $I_{n} \cup I_{n}^{q_{n}}$ e sua imagem $f(T)$ por $f$, a qual contém o valor crítico $f\left(c_{0}\right)$; note que a família $\left\{T, f(T), \ldots, f^{q_{n+1}}(T)\right\}$ tem intersecção de multiplicidade igual a 3. Observamos a distorção do cross-ratio de $f^{q_{n+1}-1}$ no par $\left(I_{n}^{1}, f(T)\right)$. Pela desigualdade de Cross-Ratio, temos

$$
D\left(f^{q_{n+1}-1} ; I_{n}^{1}, f(T)\right)=\frac{b\left(I_{n}^{q_{n+1}}, f^{q_{n+1}}(T)\right)}{b\left(I_{n}^{1}, f(T)\right)} \leq B
$$

Mas

$$
b\left(I_{n}^{q_{n+1}}, f^{q_{n+1}}(T)\right)=\frac{\left|I_{n+1}^{q_{n+1}}\right|}{\left|I_{n+1}^{q_{n+1}}\right|+\left|I_{n}^{q_{n+1}}\right|} \cdot \frac{\left|I_{n}^{q_{n+1}+q_{n}}\right|}{\left|I_{n}^{q_{n+1}}\right|+\left|I_{n}^{q_{n+1}+q_{n}}\right|} .
$$

Como os intervalos $I_{n}^{q_{n+1}+q_{n}}$ e $I_{n}^{q_{n+1}}$ são dinâmicamente simétricos com respeito de seu ponto extremo em comum, do Lema B.1.3 dizemos que a segunda fração no lado direito de (B.2.9) é limitado por acima por $C_{3}^{-1} /\left(1+C_{3}\right)$. Os intervalos $I_{n+1}^{q_{n+1}}$ e $I_{n+1}$ também são dinâmicamente simétricos com respeito a seu ponto extremo em comum, logo novamente pelo Lema B.1.3 temos $C_{3}^{-1}\left|I_{n+1}\right| \leq\left|I_{n+1}^{q_{n+1}}\right| \leq C_{3}\left|I_{n+1}\right|$; Além disso, $I_{n+1}^{q_{n+1}} \subset I_{n+1} \cup I_{n}$, assim $\left|I_{n+1}^{q_{n+1}}\right| \leq\left|I_{n+1}\right|+\left|I_{n}\right|$. Colocando estas estimativas em (B.2.9), obtemos que

$$
b\left(I_{n}^{q_{n+1}}, f^{q_{n+1}}(T)\right) \geq \theta_{1} \frac{\left|I_{n+1}\right|}{\left|I_{n}\right|},
$$

onde $\theta_{1}=C_{3}^{-2}\left(1+C_{3}\right)^{-1}\left(1+C_{3}+C_{3}^{2}+C_{3}^{3}\right)^{-1}$. Isto limita o numerador de (B.2.8) por baixo, agora só falta limitar o denominador por acima. Temos que

$$
b\left(I_{n}^{1}, f(T)\right)=\frac{\left|I_{n+1}^{1}\right|}{\left|I_{n+1}^{1}\right|+\left|I_{n}^{1}\right|} \cdot \frac{\left|I_{n}^{1+q_{n}}\right|}{\left|I_{n}^{1}\right|+\left|I_{n}^{1+q_{n}}\right|} .
$$

Como os intervalos $I_{n}^{1}$ e $I_{n}^{1+q_{n}}$ também são dinâmicamente simétricos com respeito do seu ponto extremal em comum, então usando o Lema B.1.3 mas uma vez obtemos

$$
b\left(I_{n}^{1}, f(T)\right) \leq \frac{C_{3}}{1+C_{3}} \frac{\left|I_{n+1}^{1}\right|}{\left|I_{n}^{1}\right|}
$$

Aqui, usando a propriedade de power-law no ponto crítico, temos que

$$
\frac{\left|I_{n+1}^{1}\right|}{\left|I_{n}^{1}\right|} \leq \gamma_{0}\left(\frac{\left|I_{n+1}\right|}{\left|I_{n}\right|}\right)^{d_{0}}
$$

onde $\gamma_{0}>0$ é a constante dada no Lema 2.2 .1 e $d_{0}>1$ é a criticalidade do ponto crítico $c_{0}$. Levando isto na equação (B.2.12) podemos dizer que

$$
b\left(I_{n}^{1}, f(T)\right) \leq \theta_{2}\left(\frac{\left|I_{n+1}\right|}{\left|I_{n}\right|}\right)^{d_{0}},
$$


onde $\theta_{2}=\gamma_{0} C_{3} /\left(1+C_{3}\right)$. Combinando as equações (B.2.10) e (B.2.13) obtemos

$$
\frac{\left|I_{n+1}\right|}{\left|I_{n}\right|} \geq\left(\frac{\theta_{1}}{B \theta_{2}}\right)^{\frac{1}{s_{0}-1}}=\theta_{3}
$$

Resumindo, temos provado que

$$
\theta_{3}\left|I_{n}\right| \leq\left|I_{n+1}\right| \leq C_{3}\left|I_{n}\right|
$$

(v) Os intervalos $I_{n}$ e $I_{n+1}^{q_{n}}$ são comparáveis. Como $I_{n+1}^{q_{n}} \subset I_{n}$, então $\left|I_{n+1}^{q_{n}}\right| \leq\left|I_{n}\right|$. Só falta provar a desigualdade na outra direção. Para isto, consideremos o intervalo $T^{*}=I_{n+1}^{q_{n}} \cup I_{n}^{q_{n}} \cup I_{n}^{2 q_{n}}$. Vamos dar uma olhada na distorção do cross-ratio do par $\left(I_{n}^{q_{n}}, T^{*}\right)$ pelo mapa $f^{q_{n+1}-q_{n}}$. Como a família $\left\{T^{*}, f\left(T^{*}\right), \ldots, f^{q_{n+1}-q_{n}}\left(T^{*}\right)\right\}$ tem multiplicidade de intersecção igual máximo 3 , então pela Desigualdade de Cross-Ratio, temos

$$
D\left(f^{q_{n+1}-q_{n}} ; I_{n}^{q_{n}}, T^{*}\right)=\frac{b\left(I_{n}^{q_{n+1}}, f^{q_{n+1}-q_{n}}\left(T^{*}\right)\right)}{b\left(I_{n}^{q_{n}}, T^{*}\right)} \leq B
$$

Os intervalos $I_{n+1}^{q_{n+1}}$ e $I_{n+1}$ são dinâmicamente simétricos com respeito de seu ponto extremal em comum $f^{q_{n+1}}\left(c_{0}\right)$. Além disso, os intervalos $f^{q_{n+1}-q_{n}}\left(I_{n}^{2 q_{n}}\right)=I_{n}^{q_{n+1}+q_{n}}$ e $I_{n}^{q_{n+1}}$ são dinamicamente simétricos a respeito do ponto $f^{q_{n+1}+q_{n}}\left(c_{0}\right)$. Aliás, temos que $I_{n}^{q_{n+1}} \subset I_{n} \cup I_{n+1}$. Combinando estes fatos com a equação (B.2.14) e Lema B.1.3, e depois de alguns cálculos podemos concluir que

$$
b\left(I_{n}^{q_{n+1}}, f^{q_{n+1}-q_{n}}\left(T^{*}\right)\right)=\frac{\left|I_{n+1}^{q_{n+1}}\right|}{\left|I_{n+1}^{q_{n+1}}\right|+\left|I_{n}^{q_{n+1}}\right|} \frac{\left|I_{n}^{q_{n+1}+q_{n}}\right|}{\left|I_{n}^{q_{n+1}}\right|+\left|I_{n}^{q_{n+1}+q_{n}}\right|} \geq \frac{C_{3}^{-2} \theta_{3}}{\left(1+C_{3}\right)\left(1+C_{3}+C_{3}^{2}\right)} .
$$

Vamos limitar por cima o denominador na equação (B.2.15). Como os intervalos $I_{n}^{q_{n}}$ e $I_{n}^{2 q_{n}}$ são dinamicamente simétricos a respeito do ponto $f^{q_{n}}\left(c_{0}\right)$, então aplicando o Lema B.1.3, obtemos

$$
b\left(I_{n}^{q_{n}}, T^{*}\right)=\frac{\left|I_{n+1}^{q_{n}}\right|}{\left|I_{n+1}^{q_{n}}\right|+\left|I_{n}^{q_{n}}\right|} \frac{\left|I_{n}^{2 q_{n}}\right|}{\left|I_{n}^{q_{n}}\right|+\left|I_{n}^{2 q_{n}}\right|} \leq \frac{\left|I_{n+1}^{q_{n}}\right|}{\left|I_{n}^{q_{n}}\right|} \frac{C_{3}}{1+C_{3}^{-1}} \leq \frac{C_{3}^{2}}{1+C_{3}^{-1}} \frac{\left|I_{n+1}^{q_{n}}\right|}{\left|I_{n}\right|} .
$$

Juntando (B.2.16) e (B.2.16) novamente em (B.2.15), finalmente obtemos

$$
\theta_{4}\left|I_{n}\right| \leq\left|I_{n+1}^{q_{n}}\right| \leq\left|I_{n}\right|
$$

onde

$$
\theta_{4}=\frac{\left(1+C_{3}^{-1}\right) C_{3}^{-4} \theta_{3}}{B\left(1+C_{3}\right)\left(1+C_{3}+C_{3}^{2}\right)}
$$

As desigualdades (B.2.2), (B.2.3), (B.2.6), (B.2.14) e (B.2.16), proporcionam cotas para 5 dos 15 quocientes envolvidos em (B.2.1). Os outros 10 são obtidos como produtos de máximo 4 dos 5 quocientes. Definindo $K=\max \left\{C_{3}\left(C_{3}+1\right), \theta_{3}^{-1}, \theta_{4}^{-1}\right\}$, obtemos que as 15 desigualdades em (B.2.1) são validas para $C_{4}=K^{4}$. 


\section{B.3 Prova do Teorema 2.2.2}

Para provar o Teorema 2.2.2 vamos usar a Desigualdade de Cross-Ratio para propagar as desigualdades obtidas na Proposição B.2.1 para qualquer par de intervalos adjacentes de $\mathcal{P}_{n}$. Seja $M \in \mathcal{P}_{n}$, e $L, R$ seus intervalos adjacentes nessa partição. Vamos chamar $T=L \cup M \cup R$, logo só temos que provar que o b-cross-ratio $b(M, T)$ é limitado por baixo por uma constante dependendo da constante $C_{4}$ na Proposição B.2.1. Se $M$ é um intervalo curto, existe $j<q_{n}$ tal que $M=I_{n+1}^{j}$ e isto implica que os intervalos $L$ e $R$ são os dois intervalos longos. Suponhamos que $R$ é o intervalo $I_{n}^{j}$ e $L=I_{n}^{j+q_{n+1}-q_{n}}$. Como $f^{q_{n}-j}$ é um homeomorfismo que leva o intervalo $M$ sob o intervalo $M^{*}=I_{n+1}^{q_{n}}$ e o intervalo $T$ sob $T^{*}=I_{n}^{q_{n+1}} \cup I_{n+1}^{q_{n}} \cup I_{n}^{q_{n}}$, então pela Proposição B.2.1, o cross-ratio $b\left(M^{*}, T^{*}\right)$ é limitado por baixo (por uma constante que só depende de $C_{4}$ ). Como a família $\left\{T, f(T), \ldots, f^{q_{n}-j}(T)=T^{*}\right\}$ tem multiplicidade de intersecção máximo 3, pela Desigualdade de Cross-Ratio temos que

$$
D\left(f^{q_{n}-j} ; M, T\right)=\frac{b\left(M^{*}, T^{*}\right)}{b(M, T)} \leq B
$$

Por outro lado, como $\left|I_{n}^{q_{n+1}}\right| \asymp\left|I_{n+1}^{q_{n}}\right| \asymp\left|I_{n}^{q_{n}}\right|$, então $b(M, T)$ é limitado por baixo (por uma constante que depende de $\left.C_{4}\right)$. Assim, existe uma constante $C_{5}$ que depende de $C_{4}$ tal que

$$
\left(1+\frac{\left|I_{n+1}^{j}\right|}{\left|I_{n}^{j+q_{n+1}-q_{n}}\right|}\right)\left(1+\frac{\left|I_{n+1}^{j}\right|}{\left|I_{n}^{j}\right|}\right)=\frac{\left|I_{n}^{j+q_{n+1}-q_{n}} \cup I_{n+1}^{j}\right|}{\left|I_{n}^{j+q_{n+1}-q_{n}}\right|} \frac{\left|I_{n}^{j} \cup \cup I_{n+1}^{j}\right|}{\left|I_{n}^{j}\right|} \leq C_{5},
$$

isto implica que $\left|I_{n}^{j+q_{n+1}-q_{n}}\right| \asymp\left|I_{n+1}^{j}\right| \asymp\left|I_{n}^{j}\right|$, com constante de comparabilidade dependendo de $C_{4}$. Observemos que o mesmo argumento é válido quando $M$ é um intervalo longo. 


\section{Apêndice C}

\section{Mapas quase-simétricos}

Neste apêndice recapitulamos algumas propriedades dos mapas quase-simétricos e provamos a Proposição 3.0.1, critério chave usado para provar o Teorema 3.0.1 do capítulo 3.

Definição C.0.1. Um homeomorfismo da reta que preserva orientação $h: I \rightarrow I$ é quase-simétrico se existe uma constante $K \geq 1$ tal que, para todo $x \in I$ e todo $t>0$, temos

$$
\frac{1}{K} \leq \frac{|h(x+t)-h(x)|}{|h(x)-h(x-t)|} \leq K
$$

Lema C.0.1. Seja h homeomorfismo quase-simétrico com constante de quase-simetria dada por $K \geq 1$, então h é Hölder com exponente

$$
\alpha=\frac{\log ((K+1) / K)}{\log 2} .
$$

Prova do Lema C.0.1. (Veja [GH02, Página 462] ou [Ahl66]) Observemos que pre e post composição de um mapa quase-simétrico por mapas afines é novamente um mapa quase-simétrico com mesma constante de quase-simetria. Assim, podemos supor que $h(0)=0$ e $h(1)=1$. Seja $x=1 / 2$ e $t=1 / 2$. Pela desigualdade (C.0.1) temos

$$
\frac{1}{K+1} \leq h\left(\frac{1}{2}\right) \leq \frac{K}{K+1}
$$

Portanto, para todo $n \geq 0$,

$$
\frac{1}{(K+1)^{n}} \leq h\left(\frac{1}{2^{n}}\right) \leq\left(\frac{K}{K+1}\right)^{n} .
$$

Como $h$ é monótona, se $\frac{1}{2^{n}} \leq x \leq \frac{1}{2^{n-1}}$ então

$$
h(x) \leq\left(\frac{K}{K+1}\right)^{n-1}=\left(\frac{K+1}{K}\right)\left(\frac{K}{K+1}\right)^{n}:=C\left(\frac{K}{K+1}\right)^{n} .
$$

Como $1 / 2^{n} \leq x$, temos que $-n \log 2 \leq \log x$. Observemos as seguintes igualdades

$$
-n \log 2=\frac{n \log 2 \cdot \log (K /(K+1))}{-\log (K /(K+1))}=\frac{n \log 2 \cdot \log (K /(K+1))}{\log ((K+1) / K)}=\frac{\log 2 \cdot \log (K /(K+1))^{n}}{\log ((K+1) / K)} .
$$


Então,

$$
\log x \geq \frac{\log (K /(K+1))^{n}}{\left(\frac{\log ((K+1) / K)}{\log 2}\right)},
$$

ou seja, $\log x^{\frac{\log (K+1 / K)}{\log 2}} \geq \log \left(\frac{K}{K+1}\right)^{n}$. Consequentemente, $h(x) \leq C x^{\alpha}$ para $\alpha=\frac{\log ((K+1) / K)}{\log 2}$.

O principal interesse neste tipo de mapas é que estes são restrições de mapas quase-conformes no semiplano superior. Reciprocamente, a restrição de todo mapa quase-conforme do semiplano superior na reta é um mapa quase-simétrico (veja [Ahl66] e [AB56]).

\section{C.1 Prova da Proposição 3.0.1}

Nesta subseção vamos provar a Proposição 3.0.1. Para isso vamos precisar do seguinte lema

Lema C.1.1. Seja $\left\{\mathcal{Q}_{n}\right\}_{n \geq 0}$ uma fine grid ${ }^{1}$ com constantes a e $\rho$. Seja $I \subset S^{1}$ um intervalo com interior não vazio, e seja $n=n(I)$ o menor número natural tal que $I \supset \Delta$ para algum átomo $\Delta \in \mathcal{Q}_{n}$. Então existe um intervalo $U \supset I$ que satisfaz as seguintes duas propriedades:

(i) U é união de máximo 2 a intervalos de $\mathcal{Q}_{n}$;

(ii) $|U| \leq \alpha^{-1}(1+\rho)|I|$, onde $\alpha$ é a constante em (3.0.1).

Prova do Lema C.1.1. Suponhamos que $I$ interseta 3 intervalos diferentes e consecutivos de $\mathcal{Q}_{n-1}$, digamos $\Delta_{1}, \Delta_{2}, \Delta_{3}$, onde $\Delta_{2}$ fica entre $\Delta_{1}$ e $\Delta_{3}$. Então $\Delta_{2} \subseteq I$; mas isto contradiz a definição de $n=n(I)$. Portanto, $I$ deve estar contido na união $U$ de máximo dois intervalos de $\mathcal{Q}_{n-1}$. Como as $\mathcal{Q}_{n}$ criam uma fine grid, cada átomo de $\mathcal{Q}_{n-1}$ é união de máximo $a$ intervalos de $\mathcal{Q}_{n}$, então item (i) é verdadeiro. Só falta provar item (ii). Dado que $I \supset \Delta \in \mathcal{Q}_{n}$, seja $\Delta^{*}$ o único átomo de $\mathcal{Q}_{n-1}$ que contém $\Delta$. Pelo item (i), $U$ contém $\Delta^{*}$ e máximo mais um outro átomo $\Delta^{* *} \in \mathcal{Q}_{n-1}$ adjacente a $\Delta^{*}$. Pela propriedade (c) da definição de fine grid 3.0.3 e pela desigualdade (3.0.1) no capítulo 3, obtemos

$$
|U| \leq\left|\Delta^{*}\right|+\left|\Delta^{* *}\right| \leq(1+\rho)\left|\Delta^{*}\right| \leq \alpha^{-1}(1+\rho)|\Delta| \leq \alpha^{-1}(1+\rho)|I| .
$$

Prova da Proposição 3.0.1. Precisamos verificar a seguinte condição

$$
\frac{1}{K} \leq \frac{h(x+t)-h(x)}{h(x)-h(x-t)} \leq K
$$

para todo $x \in S^{1}=\mathbb{R} / \mathbb{Z}$ e $t>0$, e para alguma constante $K>1$. Seja $I=[x-t, x+t]$ o intervalo do círculo que contém o ponto $x, \operatorname{com} I=I^{-} \cup I^{+}$, onde $I^{-}=[x-t, x]$ e $I^{+}=[x, x+t]$. Pelo Lema C.1.1, existe $n=n(I)$ e um intervalo $U \supset I$ tal que $U$ está no interior de máximo $2 a$ intervalos de $\mathcal{Q}_{n}$ e $|U| \leq \rho_{1}|I|$, onde $\rho_{1}=\alpha^{-1}(1+\rho)$. Seja $p$ o menor inteiro positivo tal que $\beta^{p} \rho_{1}<\frac{1}{4}$. Escrevemos $U$ como a união de intervalos de $\mathcal{Q}_{n+p}$, ou seja,

$$
U=J_{1} \cup J_{2} \cup \cdots \cup J_{s},
$$

onde $J_{i} \in \mathcal{Q}_{n+p}, 1 \leq i \leq s$ estão ordenados no sentido anti-horário sobre o círculo. Note que $s \leq 2 a^{p+1}$. Por (3.0.1) e indução, temos que $\left|J_{i}\right| \leq \beta^{p}\left|J_{i}^{*}\right|$, onde $J_{i}^{*} \subseteq U$ é união de um único intervalo de $\mathcal{Q}_{n}$ que

\footnotetext{
${ }^{1}$ Veja capítulo 3 definição 3.0.3.
} 
contém $J_{i}$. Consequentemente obtemos

$$
\left|J_{i}\right| \leq \beta^{p}\left|J_{i}^{*}\right| \leq \beta^{p}|U| \leq \beta^{p} \rho_{1}|I|<\frac{1}{4}|I| .
$$

Mas isto significa que pelo menos um dos intervalos $J_{i}$ 's, digamos $J_{i_{0}}$, está contido em $I^{-}$. Então, temos que $J_{i_{0}} \subset I^{-}$e que $I^{+} \subseteq J_{i_{0}+1} \cup J_{i_{0}+2} \cup \cdots \cup J_{s}$. Aliás, pela hipótese (3.0.2), para todo $1 \leq i \leq s-1$ temos

$$
\frac{\left|h\left(J_{i+1}\right)\right|}{\left|h\left(J_{i}\right)\right|} \leq \lambda+\frac{\left|J_{i+1}\right|}{\left|J_{i}\right|} \leq \lambda+\rho,
$$

de isto segue-se que

$$
\frac{\left|h\left(J_{i+\nu}\right)\right|}{\left|h\left(J_{i}\right)\right|} \leq(\lambda+\rho)^{\nu} \text { for all } \nu=1,2, \cdots, s-i
$$

Portanto

$$
\frac{h(x+t)-h(x)}{h(x)-h(x-t)}=\frac{\left|h\left(I^{+}\right)\right|}{\left|h\left(I^{-}\right)\right|} \leq \frac{\sum_{i=i_{0}+1}^{s}\left|h\left(J_{i}\right)\right|}{\left|h\left(J_{i_{0}}\right)\right|} \leq \sum_{\nu=1}^{s-i_{0}}(\lambda+\rho)^{\nu} \leq \sum_{\nu=1}^{2 a^{p+1}}(\lambda+\rho)^{\nu} .
$$

Isto prova que $h$ é $K$-quase-simétrica com constante de quase-simetria $K=\sum_{\nu=1}^{2 a^{p+1}}(\lambda+\rho)^{\nu}$, a qual só depende das constantes $a, \rho, \lambda$ da fine grid. 
68 APÊNDICE C 


\section{Referências Bibliográficas}

[AB56] L. Ahlfors e A. Beurling. The boundary correspondence under quasiconformal mappings. Acta Mathematica, 96(1):125-142, 1956. 66

[Ahl66] L. Ahlfors. Lectures on Quasiconformal Mappings. Van Nostrand, 1966. 65, 66

[Arn61] V. Arnol'd. Small denominators i. mappings of the circle onto itself. Izv. Akad. Nauk. Math. Series, 25:21-86, 1961. [Translations of the Amer. Math. Soc. (series 2), 46, 213284, (1965)]. 1, 6

[Avi13] A. Avila. On rigidity of critical circle maps. Bull. Braz. Math. Soc., páginas 611-619, 2013. 2, 6, 7, 13, 45, 46

[BBJ84] T. Bohr, P. Bak e M. Jensen. Transition to chaos by interaction of resonances in dissipative systems. ii. josephson junctions, charge-density waves, and standard maps. Physical review A, 30(4):1970, 1984.2

[Car67] L. Carleson. On mappings conformal at the boundary. J. Anal. Math., 19:1-13, 1967. 19

[CvS14] T. Clark e S. van Strien. Quasisymmetric rigidity in one-dimensional dynamics. Preprint, 2014. 3, 6, 7

[Den32] A. Denjoy. Sur les courbes définies par les équations différentielle à la surface du tore. J. Math. Pure et Appl., 11:333-376, 1932. 5

[dF99] E. de Faria. Asymptotic rigidity of scaling ratios for critical circle mappings. Ergod. Th. \& Dynam. Sys., 19:995-1035, 1999. 6, 7, 45

[dFdM99] E. de Faria e W. de Melo. Rigidity of critical circle mappings i. J. Eur. Math. Soc., 1:339-392, 1999. 2, 3, 4, 6, 7, 11, 13, 19, 25, 26, 30, 33, 37, 40, 41, 42, 45, 46, 57

[dFdM00] E. de Faria e W. de Melo. Rigidity of critical circle mappings ii. J. Amer. Math. Soc., 13:343-370, 2000. 2, 6, 7, 13

[dFG] E. de Faria e P. Guarino. Counterexample for the quasisymmetric conjugacy between multicritical circle maps. In progress. 35

[dFG16] E. de Faria e P. Guarino. Real bounds and lyapunov exponents. Discrete and Continuous Dynamical Systems A, 36:1957-1982, 2016. 12

[dM98] W. de Melo. Rigidity and renormalization in the one dimensional dynamical systems. Proceedings of the International Congress of Mathematicians, Berlin, Doc. Math. Vol II:765779, 1998. 16 
[dM11] W. de Melo. Dynamics on the circle. Em Springer, editor, Dynamics, Games and Science I, páginas 651-659. 2011. 6, 45

[dMvS93] W. de Melo e S. van Strien. One dimensional dynamics. Springer-Verlag, 1993. 5, 19, 47, $50,53,54$

[dSG10] H. de St Gervais. Uniformisation des surfaces de Riemann: retour sur un théoreme centenaire. ENS, 2010. 53

[EdF15] G. Estevez e E. de Faria. Real bounds and quasisymmetric rigidity of multicritical circle maps. To appear in Trans. Amer. Soc., avaliable at arXiv:1511.09056, 2015. 3, 19, 44, 57

[EdFG16] G. Estevez, E. de Faria e P. Guarino. Beau bounds for multicritical circle maps. arXiv:1611.00722, 2016. 3, 37

[GdM13] P. Guarino e W. de Melo. Rigidity of smooth critical circle maps. Available at arXiv:1303.3470, 2013. 2, 13

[GH02] F. Gardiner e W. Harvey. Handbook of Complex Analysis: Geometric function theory, volume 1. North-Holland, 2002. 65

[Gla91] L. Glass. Cardiac arrhythmias and circle maps- a classical problem. Chaos: An Interdisciplinary Journal of Nonlinear Science, 1(1):13-19, 1991. 2

[GMdM15] P. Guarino, M. Martens e W. de Melo. Rigidity of critical circle maps. Available at arXiv:1511.02792, 2015. 2, 6, 7, 13

[Gua12] P. Guarino. Rigidity Conjecture for C3 Critical Circle Maps. Tese de Doutorado, IMPA, Brasil, 2012. 7, 13, 49, 55

[Gua15] P. Guarino. Some topological and geometrical aspects of circle dynamics. Cadernos de Matemática, ICMC-USP, 16(1):39-69, 2015. 7

[Hal81] G. Hall. A c1 denjoy counterexample. Ergod. Th. and Dyna. Sys., 1:261-272, 1981. 2, 7

[Her79] M. Herman. Sur la conjugaison différentiable des difféomorphismes du cercle à des rotations. Publ. Math. IHES, 49:5-233, 1979. 1

[Her88] M. Herman. Conjugaison quasi-simétrique des homéomorphismes du cercle à des rotations. Manuscript, 1988. 4, 11

[KH] A. Katok e B. Hasselblatt. Introduction to the modern theory of dynamical systems. 47, 49

[Kha91] K. Khanin. Universal estimates for critical circle mappings. Chaos, 1(2):181-186, 1991. 12

[Khi97] A. Khinchin. Continued fractions. Dover Publications, 2 edição, 1997. reprint of the 1964 translation. 48,49

[KS87] K. Khanin e Y. Sinai. A new proof of $m$. herman's theorem. Commun. Math. Phys., 112:89-101, 198\%. 1

[KS89] K. Khanin e Y. Sinai. Smoothness of conjugacies of diffeomorphisms of the circle with rotations. Russ. Math. Surv., 44(1):69-99, 1989. 1 
[KT07] K. Khanin e A. Teplinsky. Robust rigidity for circle diffeomorphisms with singularities. Invent. Math., 169:193-218, 2007. 2, 13, 45

[KT09] K. Khanin e A. Teplinsky. Herman's theory revisited. Invent. Math., 178:333-344, 2009. 1

[KY06] D. Khmelev e M. Yampolsky. The rigidity problem for analytic critical circle maps. Mosc. Math. J., 6:317-351, 2006. 6, 7, 13

[Lag79] J. Lagrange. Sur la constructions des cartes géographiques. Nouveau Mémoire de l'Académie de Berlin, páginas 161-210, 1779. 53

[Pal15] L. Palmisano. Quasi-symmetric conjugation for circle maps with a flat interval. arXiv:1510.01703, 2015. 3, 46

[Pet00] C. Petersen. The herman-światek theorems with applications. Lectures Note Series, London Mathematical Society, 274:211-225, 2000. 4, 11

[PM80] Y. Pomeau e P. Manneville. Intermittent transition to turbulence in dissipative dynamical systems. Comm. Math. Phys., 74(2):189-197, 1980. 2

[Poi82] H. Poincaré. Mémoire sur les courbes définies par une équation différentielle (ii). Journal de mathématiques pures et appliquées, páginas 251-296, 1882. 5, 47

[Ran88] D. Rand. Global phase space universality, smooth conjugacies and renormalisation. $i$. the $c 1+\alpha$ case. Nonlinearity, 1(1):181-202, 1988. 1

[Sta88] J. Stark. Smooth conjugacy and renormalisation for diffeomorphisms of the circle. Nonlinearity, 1(4):451-575, 1988. 1

[Sul88] D. Sullivan. Bounds, quadratic differentials, and renormalization conjectures. AMS Centennial Publications, Mathematics into the Twenty-first Century, II:417-466, 1988. 3, 37, 41

[Swi88] G. Swiatek. Rational rotation numbers for maps of the circle. Comm. Math. Phys., 119:109128, 1988. 4, 6, 7, 11

[VO15] M. Viana e K. Oliveira. Foundations of Ergodic Theory. Cambridge University Press, 2015. 12

[Yam01] M. Yampolsky. The attractor of renormalization and rigidity of towers of critical circle maps. Comm. Math. Phys., 218:537-568, 2001. 6, 7

[Yam02] M. Yampolsky. Hyperbolicity of renormalization of critical circle maps. Publ. Math. IHES, 96:1-41, 2002. 6, 7

[Yam03] M. Yampolsky. Renormalization horseshoe for critical circle maps. Commun. Math. Phys., 240:75-96, 2003. 6, 7

[YK89] D. Ornstein Y. Katznelson. The differentiability of the conjugation of certain diffeomorphisms of the circle. Ergod. Th. and Dynam. Sys., 9:643-680, 1989. 1

[Yoc84a] J. C. Yoccoz. Conjugaison différentiable des difféomorphismes du cercle dont le nombre de rotation vérifie une condition diophantienne. Ann. Scient. Éc. Norm. Sup., 17:333-361, 1984. 1 
[Yoc84b] J. C. Yoccoz. Il n'y a pas de contre-exemple de denjoy analytique. C.R. Acad. Sc. Paris, 298:141-144, 1984. 2, 6, 7, 8 